\title{
SEMISTABLE HIGGS BUNDLES, PERIODIC HIGGS BUNDLES AND REPRESENTATIONS OF ALGEBRAIC FUNDAMENTAL GROUPS
}

\author{
GUITANG LAN, MAO SHENG, AND KANG ZUO
}

\begin{abstract}
Let $k$ be the algebraic closure of a finite field of odd characteristic $p$ and $X$ a smooth projective scheme over the Witt ring $W(k)$ which is geometrically connected in characteristic zero. We introduce the notion of Higgs-de Rham flou 1 and prove that the category of periodic Higgs-de Rham flows over $X / W(k)$ is equivalent to the category of Fontaine modules, hence further equivalent to the category of crystalline representations of the étale fundamental group $\pi_{1}\left(X_{K}\right)$ of the generic fiber of $X$, after Fontaine-Laffaille and Faltings. Moreover, we prove that every semistable Higgs bundle over the special fiber $X_{k}$ of $X$ of rank $\leq p$ initiates a semistable Higgs-de Rham flow and thus those of rank $\leq p-1$ with trivial Chern classes induce $k$-representations of $\pi_{1}\left(X_{K}\right)$. A fundamental construction in this paper is the inverse Cartier transform over a truncated Witt ring. In characteristic $p$, it was constructed by Ogus-Vologodsky in the nonabelian Hodge theory in positive characteristic; in the affine local case, our construction is related to the local Ogus-Vologodsky correspondence of Shiho.
\end{abstract}

\section{Contents}

1. Introduction

2. Preliminaries on Fontaine modules

3. Higgs correspondence in positive characteristic

4. Inverse Cartier transform over a truncated Witt ring 21

5. Higgs correspondence in mixed characteristic 37

6. Strongly semistable Higgs modules

7. Rigidity theorem for Fontaine modules 51

Appendix A. Semistable Higgs bundles of small ranks are strongly Higgs semistable

References

The first and third named authors are supported by the SFB/TR 45 'Periods, Moduli Spaces and Arithmetic of Algebraic Varieties' of the DFG. The second named author is supported by National Natural Science Foundation of China (Grant No. 11622109, No. 11626253) and the Fundamental Research Funds for the Central Universities.

1. Higgs-de Rham flow was called Higgs-de Rham sequence in 14. The change has been made because of its analogue to the notion of Yang-Mills-Higgs flow over $\mathbb{C}$. 


\section{INTRODUCTION}

Let $k$ be the algebraic closure of a finite field of odd characteristic $p, W:=W(k)$ the ring of Witt vectors and $K$ its fraction field. Let $X$ be a smooth projective scheme over $W$ which is geometrically connected in characteristic zero. The paper is aimed to establish a correspondence between certain Higgs bundles over $X$ with trivial Chern classes which are stable over its special fiber $X_{k}:=X \times_{W} k$ and certain integral crystalline representations which are absolutely irreducible modulo $p$ of the étale fundamental group $\pi_{1}\left(X_{K}\right)$ of the generic fiber $X_{K}:=X \times_{W} K$.

Inspired by the complex analytic theory of Simpson [31], Ogus and Vologodsky 25. has established the nonabelian Hodge theorem in positive characteristic, that is an equivalence of categories between a category of certain nilpotent Higgs modules and a category of certain nilpotent flat modules over a smooth variety over $k$ which is $W_{2}(k)$-liftable (char $k=2$ is also allowed). This equivalence generalizes both the classical Cartier descent theorem and the relation between a strict $p$-torsion Fontaine module and the associated graded Higgs module. Compared with the complex theory, an obvious distinction is that the semistability condition on Higgs modules does not play any role in Ogus-Vologodsky's correspondence. In the meantime, Faltings and others have been attempting to establish an analogue of Simpson's theory for varieties over $p$-adic fields. In [6], Faltings has established an equivalence of categories between the category of generalized representations of the geometric fundamental group and the category of Higgs bundles over a $p$-adic curve, which has generalized the earlier work of DeningerWerner [2] on a partial p-adic analogue of Narasimhan-Seshadri theory. However, the major problem concerning the role of semistability remains open; Faltings asked whether semistable Higgs bundles of degree zero come from genuine representations instead of merely generalized ones. We will address the semistability condition in this paper.

Recall that, when $X$ is a complex smooth projective variety, such a correspondence has been established first by Hitchin [10] for polystable Higgs bundles over a smooth projective curve and then by Simpson [30] in general. The key step in the Hitchin-Simpson correspondence, as one may see in the following diagram, is to solve the Yang-Mills-Higgs equation and obtain a correspondence from graded Higgs bundles to polarized complex variations of Hodge structure over $X$.

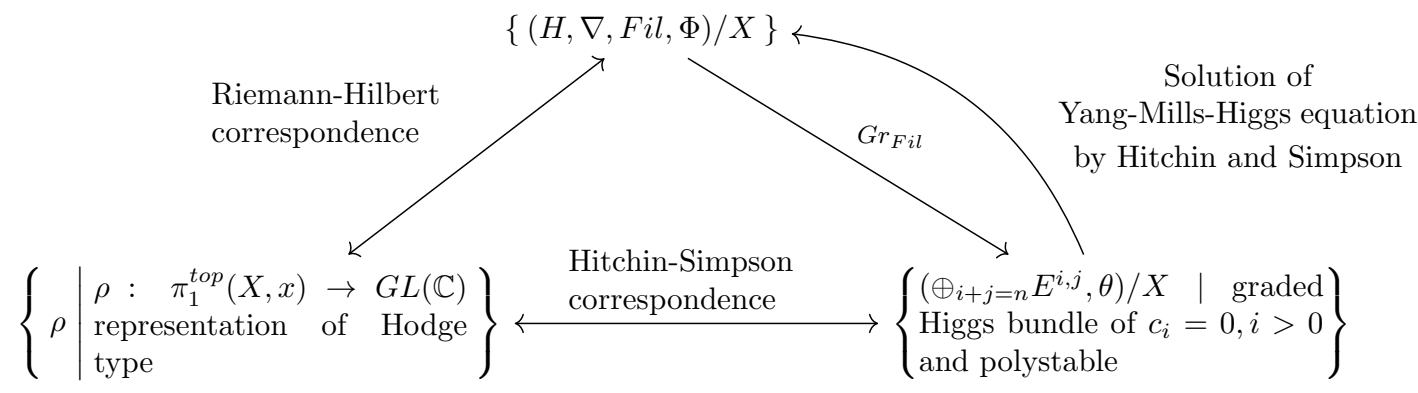


The notion of polarized $\mathbb{Z}$-variation of Hodge structure $(H, \nabla, F i l, \Phi)$ was introduced by P. Griffiths [9], and later generalized to polarized $\mathbb{C}$-variation of Hodge structure by P. Deligne [1], where $(H, \nabla)$ is a flat bundle with a Hodge filtration, $\Phi$ is a polarization, which is horizontal with respect to $\nabla$ and satisfies the RiemannHodge bilinear relations; $\left(\oplus_{i+j=n} E^{i, j}, \theta\right)$ denotes a graded Higgs bundle, where $\theta$ is a direct sum of $\mathcal{O}_{X^{-}}$linear morphisms $\theta^{i, j}: E^{i, j} \rightarrow E^{i-1, j+1} \otimes \Omega_{X}$ and $\theta \wedge \theta=0$.

We return to the $p$-adic case. A good $p$-adic analogue of the category of polarized complex variations of Hodge structure is the category $M F_{[0, w]}^{\nabla}(X / W)(w \leq p-1)$ introduced first by Fontaine-Laffaille [7] for $X=$ Spec $W$ and later generalized by Faltings [4, Chapter II] to the general case. An object in the category, called Fontaine module, is also a quadruple $(H, \nabla, F i l, \Phi)$, where $(H, \nabla)$ is a flat bundle over $X$, by which we mean a locally free $\mathcal{O}_{X}$-module $H$ of finite rank, equipped with an integrable $W$-connection $\nabla, F i l$ is a Hodge filtration, $\Phi$ is a relative Frobenius which is horizontal with respect to $\nabla$ and satisfies some compatibility and strong $p$-divisibility conditions. The latter condition is a $p$-adic analogue of the Riemann-Hodge bilinear relations. See $\S 2$ for more details. Fontaine-Laffaille proved in the $X=$ Spec $W$ case and Faltings in the general case that, there exists a fully faithful functor from the category $M F_{[0, w]}^{\nabla}(X / W)(w \leq p-2)$ to the category of crystalline representations of $\pi_{1}\left(X_{K}\right)$, i.e. a $p$-adic Riemann-Hilbert correspondence. Our objective is to establish a $p$-adic analogue of the Higgs correspondence from a certain category of graded Higgs modules with trivial Chern classes to the category of Fontaine modules. Our results are encoded in the following big diagram:

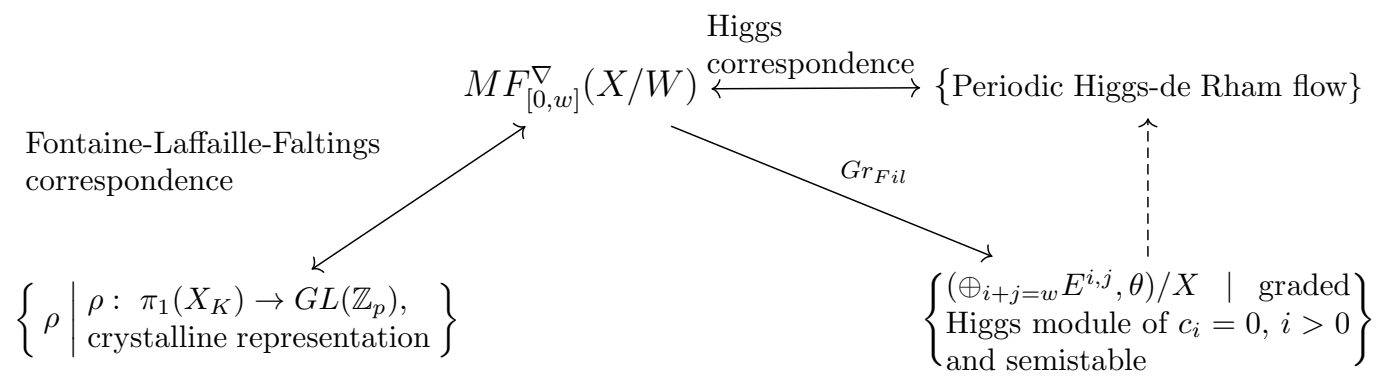

As seen from the above diagram, the central notion in our theory is Higgs-de Rham flow (especially the periodic one), which can be viewed as an analogue of Yang-Mills-Higgs flow. To define a Higgs-de Rham flow over $X_{n}:=X \otimes \mathbb{Z} / p^{n} \mathbb{Z}$ for all $n \in \mathbb{N}$, the key ingredient is the inverse Cartier transform $C_{n}^{-1}$ over $W_{n}:=W \otimes \mathbb{Z} / p^{n} \mathbb{Z}$, see Definition 5.1. It is built upon the seminal work of Ogus-Vologodsky [25]. In [25], they construct the inverse Cartier transform from the category of (suitably nilpotent) Higgs modules over $X_{1}^{\prime}=X_{1} \times_{F_{k}}$ Spec $k$ to the category of (suitably nilpotent) flat modules over $X_{1}$. However, over a general smooth scheme $X^{\prime}$ over $W_{n}, n \geq 2$, our lifting of inverse Cartier transform operates not on the whole category of (suitably nilpotent) Higgs modules, rather on a category which is over a subcategory of graded Higgs modules (over a proper scheme, there is a restriction on the Chern classes of Higgs modules). More details 
about the category will be given below. In comparison with the construction of Shiho [29], one finds that the existence of a Frobenius lifting over a chosen lifting of $X^{\prime}$ over $W_{n+1}$ is not assumed in our construction. On the other hand, we do not know whether the functor $C_{n}^{-1}$ is fully faithful for a proper $W_{n}$-scheme when $n \geq 2$.

The Higgs correspondence is established in an inductive way. That is, we shall first define the notion of a Higgs de-Rham flow in characteristic $p$ and then establish the Higgs correspondence for the periodic flows. This is the first step. In this step, we need only assume the scheme $X_{1}$ to be smooth over $k$ and $W_{2^{-}}$ liftable. A choice of such a lifting does matter in the theory. Let us choose and then fix a lifting $X_{2} / W_{2}$. Let $S_{n}:=$ Spec $W_{n}$ and $F_{S_{n}}: S_{n} \rightarrow S_{n}$ the Frobenius automorphism. Set $X_{2}^{\prime}:=X_{2} \times_{F_{S_{2}}} S_{2}$ which is a $W_{2}$-lifting of $X_{1}^{\prime}=X_{1} \times_{F_{S_{1}}} S_{1}$. Let $(\mathcal{X}, \mathcal{S})=\left(X_{1} / k, X_{2}^{\prime} / W_{2}\right)$ and $C_{\mathcal{X} / \mathcal{S}}^{-1}$ the inverse Cartier transform of OgusVologodsky [25] which restricts to an equivalence of categories from the full subcategory of nilpotent Higgs modules $H I G_{p-1}\left(X_{1}^{\prime}\right)$ of exponent $\leq p-1$ to the full subcategory of nilpotent flat modules $M I C_{p-1}\left(X_{1}\right)$ of exponent $\leq p-1$. See also our previous work [13] for an alternative approach via the exponential twisting to the Ogus-Vologodsky's theory over these subcategories. Let $\pi: X_{1}^{\prime} \rightarrow X_{1}$ be the base change of $F_{S_{1}}$ to $X_{1}$. From the geometric point of view, it is more natural to make all terms in a flow defining over the same base scheme. So, instead of using $C_{\mathcal{X} / \mathcal{S}}^{-1}$, we take the composite functor $C_{1}^{-1}:=C_{\mathcal{X} / \mathcal{S}}^{-1} \circ \pi^{*} \circ \iota$ from $H I G_{p-1}\left(X_{1}\right)$ to $M I C_{p-1}\left(X_{1}\right)$, where $\iota$ is an automorphism of the category $H I G_{p-1}\left(X_{1}\right)$ defined by sending $(E, \theta)$ to $(E,-\theta)$. The reader is advised to take caution on this point. For a flat module $(H, \nabla)$ over $X_{1}$, a Griffiths transverse filtration Fil of level $w \geq 0$ on $(H, \nabla)$ is defined to be a finite exhaustive decreasing filtration of $H$ by $\mathcal{O}_{X_{1}}$-submodules

$$
H=F i l^{0} \supset F i l^{1} \supset \cdots \supset F i l^{w} \supset F i l^{w+1}=0,
$$

such that its grading $\oplus_{i} F i l^{i} / F i l^{i+1}$ is torsion free and such that $F i l$ obeys Griffiths' transversality

$$
\nabla\left(F i l^{i}\right) \subset F i l^{i-1} \otimes \Omega_{X_{1} / k}, \quad 1 \leq i \leq w .
$$

The triple $(H, \nabla, F i l)$ is called a de Rham module. By taking the grading with respect to the filtration $F i l$, to every de Rham module $(H, \nabla, F i l)$ one can canonically associate a graded Higgs module $(E, \theta):=\left(\oplus_{i} F i l^{i} / F i l^{i+1}, \oplus_{i} \bar{\nabla}_{i}\right)$, where the $\mathcal{O}_{X_{1}}$-morphism $\bar{\nabla}_{i}$ is induced from $\nabla$

$$
\bar{\nabla}_{i}: \frac{F i l^{i}}{F i l^{i+1}} \rightarrow \frac{F i l^{i-1}}{F i l^{i}} \otimes \Omega_{X_{1} / k}
$$


Definition 1.1. A Higgs-de Rham flow over $X_{1}$ is a diagram of the following form:

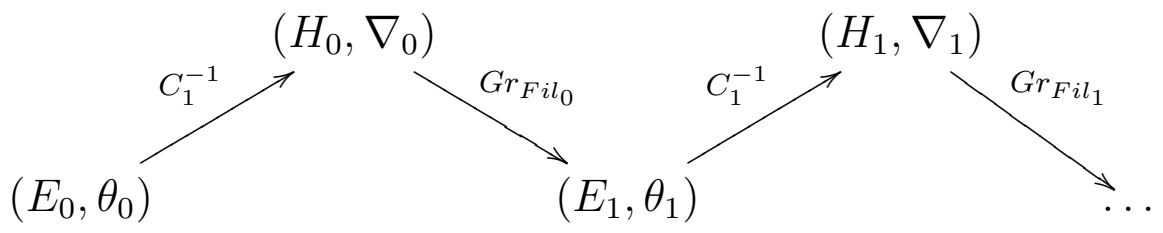

where the initial term $\left(E_{0}, \theta_{0}\right) \in H I G_{p-1}\left(X_{1}\right), F i l_{i}, i \geq 0$ is a Griffiths transverse filtration on the flat module $\left(H_{i}, \nabla_{i}\right):=C_{1}^{-1}\left(E_{i}, \theta_{i}\right)$ of level $\leq p-1$ and $\left(E_{i}, \theta_{i}\right), i \geq 1$ is the associated graded Higgs module to the de Rham module $\left(H_{i-1}, \nabla_{i-1}, F i l_{i-1}\right)$.

If the filtrations $F i l_{i}, i \geq 0$ in the definition are all of level $\leq w(w \leq p-1)$, it is said to be a Higgs-de Rham flow of level $\leq w$. For a graded torsion-free Higgs module $\left(E=\oplus_{i} E^{i}, \theta\right) \in H I G_{p-1}\left(X_{1}\right)$ (where $\left.\theta: E^{i} \rightarrow E^{i-1} \otimes \Omega_{X_{1} / k}\right)$, there is a natural Griffiths transverse filtration on $(H, \nabla):=C_{1}^{-1}(E, \theta)$, which is actually nontrivial in general. Since the filtration $\left\{E_{l}:=\oplus_{i<l} E^{i}\right\}_{l}$ of $E$ is $\theta$-invariant, and since $C_{1}^{-1}$ is an exact functor, $\left\{\left(H_{l}, \nabla_{l}\right):=C_{1}^{-1}\left(E_{l}, \theta\right)\right\}_{l}$ is naturally a filtration of $(H, \nabla)$ and it is Griffiths transverse. But since $\left\{H_{l}\right\}_{l}$ is $\nabla$-invariant, the associated graded Higgs module has always the zero Higgs field. It is important to observe that there exist other nontrivial Griffiths transverse filtrations than the above one: the Hodge filtration in the geometric case (i.e. the strict $p$-torsion Fontaine modules) and the Simpson filtration in the $\nabla$-semistable case (see Theorem A.4).

A Higgs-de Rham flow is said to be periodic if there exists an isomorphism of graded Higgs modules $\phi:\left(E_{f}, \theta_{f}\right) \cong\left(E_{0}, \theta_{0}\right)$ (an explicit $\phi$ is a part of the definition); it is said to be preperiodic if it becomes periodic after removing the first few terms. A Higgs module $(E, \theta) \in H I G_{p-1}\left(X_{1}\right)$ is said to be (pre)periodic if it initiates a (pre)periodic Higgs-de Rham flow. The reader shall refer to Definition 3.1 for a precise definition. One may visualize a periodic Higgs-de Rham flow of period $f$ via the following diagram:

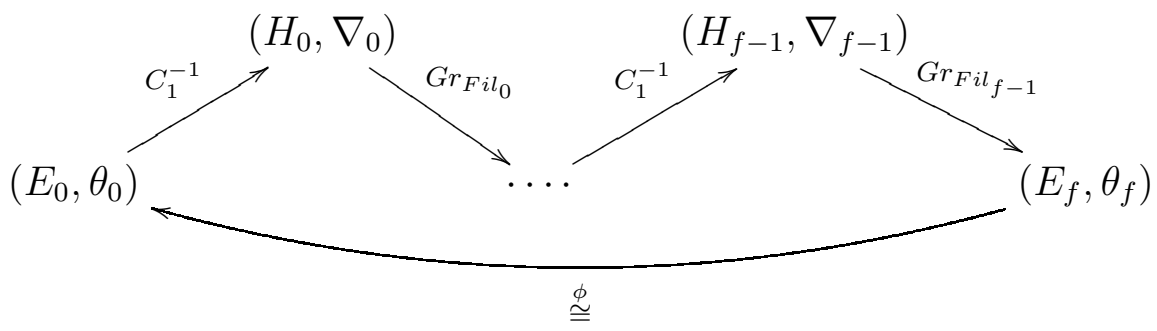

Theorem 1.2 (Theorem 3.2). Let $X_{2}$ be a smooth scheme over $W_{2}$. Let $w$ be an integer between 0 and $p-1$. For each $f \in \mathbb{N}$, there is an equivalence of categories between the full subcategory of strict p-torsion Fontaine modules over $X_{2} / W_{2}$ of Hodge-Tate weight $\leq w$ with endomorphism structure $\mathbb{F}_{p^{f}}$ and the category of periodic Higgs-de Rham flows over its special fiber $X_{1}$ of level $\leq w$ and whose periods are $f$. 
Next, we construct a lifting to $W_{n}, n \in \mathbb{N}$ of the inverse Cartier transform of Ogus-Vologodsky [25] restricted to the full subcategory $H_{I G_{p-2}}\left(X_{1}^{\prime}\right)$. Let $X_{n}$ be a smooth scheme over $W_{n}$. We introduce a category $\mathcal{H}\left(X_{n}^{\prime}\right)$, where $X_{n}^{\prime}:=X_{n} \times_{F_{S_{n}}}$ $X_{n}$, whose object is a tuple $(E, \theta, \bar{H}, \bar{\nabla}, \overline{F i l}, \bar{\psi})$, where $(E, \theta)$ is a nilpotent graded Higgs module over $X_{n}^{\prime}$ of exponent $\leq p-2,(\bar{H}, \bar{\nabla}, \bar{F} i l)$ is a de Rham module over $X_{n-1}^{\prime}$, and $\bar{\psi}$ is an isomorphism of graded Higgs modules $G r_{\overline{F i} i}(\bar{H}, \bar{\nabla}) \cong$ $(E, \theta) \otimes \mathbb{Z} / p^{n-1} \mathbb{Z}$. For $n=1$, such a tuple is reduced to a nilpotent graded Higgs module over $X_{1}^{\prime}$ of exponent $\leq p-2$, and therefore $\mathcal{H}\left(X_{1}^{\prime}\right)$ is just the full subcategory $H I G_{p-2}\left(X_{1}^{\prime}\right)$.

Theorem 1.3 (Theorem4.1). Assume $X_{n}$ is $W_{n+1}$-liftable. There exists a functor $\mathcal{C}_{n}^{-1}$ from the category $\mathcal{H}\left(X_{n}^{\prime}\right)$ to the category $\operatorname{MIC}\left(X_{n}\right)$ of flat modules over $X_{n}$ such that $\mathcal{C}_{n}^{-1}$ lifts $\mathcal{C}_{n-1}^{-1}$ and such that $\mathcal{C}_{1}^{-1}$ agrees with the inverse Cartier transform $C_{\mathcal{X} / \mathcal{S}}^{-1}$ of Ogus-Vologodsky [25].

Based on the existence of an inverse Cartier transform over $W_{n}$, we define inductively a periodic Higgs-de Rham flow over $X_{n} / W_{n}$ for an arbitrary $n$ (Definition 5.2) and establish the Higgs correspondence over $W_{n}$ (Theorem 5.3). Passing to the limit, we obtain the $p$-torsion free analogue of Theorem 1.2;

Theorem 1.4. Let $X$ be a smooth scheme over $W$. For each integer $0 \leq w \leq$ $p-2$ and each $f \in \mathbb{N}$, there is an equivalence of categories between the category of $p$-torsion free Fontaine modules over $X / W$ of Hodge-Tate weight $\leq m$ with endomorphism structure $W\left(\mathbb{F}_{p^{f}}\right)$ and the category of periodic Higgs-de Rham flows over $X$ of level $\leq w$ and whose periods are $f$.

The above theorem (and its $p$-torsion version) and the Fontaine-Laffaille-Faltings correspondence (and its $p$-torsion version, see Theorem 2.3) together constitute a $p$-adic (and $p$-torsion) version of Hitchin-Simpson correspondence. This correspondence can be regarded as a global and higher Hodge-Tate weight generalization of the Katz's correspondence on unit-root $F$-crystals. Using this correspondence, a $p$-divisible group with isomorphic Kodaira-Spencer map over $W$ is constructed over the Serre-Tate lifting of an ordinary Abelian variety over $k$. See Example 5.8,

Now, we shall bring the semistability condition on Higgs modules into consideration, and explain our results along the vertical dotted line in the above big diagram. For a smooth projective variety $X_{1}$ over $k$ of positive dimension, we choose (and then fix) an ample divisor $Z_{1}$ of $X_{1}$. Let us define the $(\mu$-)semistability for a Higgs module $(E, \theta)$ over $X_{1}$ with respect to the $\mu_{Z_{1}}$-slope:

$$
\mu_{Z_{1}}(E)=c_{1}(E) Z_{1}^{\operatorname{dim} X_{1}-1} / \operatorname{rank}(E)
$$

The slope of a torsion $\mathcal{O}_{X_{1}}$-module is set to be infinity. Our first result is the following

Theorem 1.5 (Theorem 6.6. Theorem A.1). Notation as above and assume additionally that $X_{1}$ is $W_{2}$-liftable. Let $(E, \theta)$ be a torsion free nilpotent Higgs module over $X_{1}$ of exponent $\leq p-1$. Suppose rank $E \leq p$ and $c_{i}(E)=0, i>0$. Then $(E, \theta)$ is semistable if and only if it is preperiodic. 
As a consequence, we obtain the following

Corollary 1.6 (Theorem 6.7). Let $X / W$ be a smooth projective scheme over $W$. For each nilpotent semistable Higgs bundle $(E, \theta)$ over $X_{k}$ of rank $E \leq p-1$ and $c_{i}(E)=0, i>0$, one associates to it a unique rank $E$ crystalline $k$-representation of $\pi_{1}\left(X_{K}\right)$ up to isomorphism.

The stronger rank condition in the above corollary results from the application of the Fontaine-Laffaille-Faltings correspondence.

In the way to lift the previous result to the mixed characteristic situation, a nontrivial obstruction occurs, namely the lifting of a Griffiths transverse filtration in positive characteristic to a truncated Witt ring, which has prevented us from a direct generalization of Corollary 1.6 to the mixed characteristic case. It turns out that, in order to kill the obstruction, one is led to various ordinary conditions on the base varieties. By working on this problem for a very simple kind of rank two Higgs bundles of degree zero (the so-called Higgs bundle with maximal Higgs field) over a curve, we have found a $p$-adic analogue of the Hitchin-Simpson's uniformization of hyperbolic curves which relates intimately the above theory to the theory of ordinary curves due to S. Mochizuki ([23]). In particular, the canonical lifting theorem of Mochizuki for ordinary curves has been basically recovered in our recent work [16].

Stability, rather than merely semistability, on periodic Higgs bundles over $k$ makes the choices involved in Higgs-de Rham flows basically unique. By the unicity of one-periodic Higgs-de Rham flow initializing a stable Higgs bundle (Proposition 7.2 and Proposition 7.5), one is able to identify the category of one-periodic stable Higgs modules with a full subcategory of the category of periodic Higgs-de Rham flows. Combined with this unicity, the Higgs correspondence implies the following rigidity result for Fontaine modules.

Theorem 1.7 (Corollary [.6). Let $X / W$ be a smooth projective scheme. Let $\left(H_{i}, \nabla_{i}, F i l_{i}, \Phi_{i}\right), i=1,2$ be two p-torsion free Fontaine modules over $X / W$, and $\left(E_{i}, \theta_{i}\right)$ the associated graded Higgs modules. If $\left(E_{i}, \theta_{i}\right)$ are isomorphic as graded Higgs modules and additionally mod $p$ are Higgs stable, then $\left(H_{i}, \nabla_{i}, F i l_{i}, \Phi_{i}\right), i=$ 1,2 are isomorphic.

To conclude, our final result is the following correspondence. An $\mathbb{F}_{p}$-representation $\rho$ of $\pi_{1}$ is said to be absolutely irreducible if $\rho \otimes k$ is irreducible.

Theorem 1.8 (Corollary 7.7). Notation as above. There is an equivalence of categories between the category of crystalline $\mathbb{Z}_{p}$ (resp. $\mathbb{Z} / p^{n} \mathbb{Z}$ ) representations of $\pi_{1}\left(X_{K}\right)$ with Hodge-Tate weight $\leq p-2$ whose mod $p$ reduction is absolutely irreducible and the category of one-periodic Higgs bundles over $X / W\left(\right.$ resp. $\left.X_{n} / W_{n}\right)$ whose exponent is $\leq p-2$ and $\bmod p$ reduction is stable.

In the study of Hitchin-Simpson correspondence, one usually concentrates on the subcategory of Higgs bundles with trivial Chern classes. However, the theory developed in this paper turns out to be also useful in the study of Higgs bundles with 
nontrivial Chern classes. This has been beautifully demonstrated in the recent work [20] of A. Langer on a purely algebraic proof of the Bogomolov-Giesecker inequality for semistable Higgs bundles in the complex case ([30, Proposition 3.4]) and the Miyaoka-Yau inequality for Chern numbers of complex algebraic surfaces of general type. In his work, the notion of (semistable) Higgs-de Rham flow in characteristic $p$ has played as similar role as the Yang-Mills-Higgs flow over the field of complex numbers.

The paper is structured as follows: basically, the paper consists of two parts: the first five sections $§ 2-\S 5$ are devoted to establish the theory of the Higgs correspondence between the category of $p$-torsion Fontaine modules with extra endomorphism structures and the category of periodic Higgs-de Rham flows; the last two sections $\S 6-\S 7$ and the appendix aim towards applications of the previous theory to produce the representations of $\pi_{1}$ from (semi)-stable Higgs bundles. In more detail, Section 2 is a preliminary, where we recall the basics of the theory on Fontaine modules; in Section 3, we introduce the notion of a Higgs-de Rham flow in positive characteristic and establish the Higgs correspondence in positive characteristic; in Section 4, we construct a lifting of the inverse Cartier transform of Ogus-Vologodsky over an arbitrary truncated Witt ring; in Section 5, we establish the Higgs correspondence over an arbitrary truncated ring; in Section 6 , we introduce the notion of a strongly semistable Higgs module and show that a strongly semistable Higgs module with trivial Chern classes is preperiodic and vice versa, and consequently we produce crystalline representations of the algebraic fundamental groups of the generic fiber with $k$-coefficients from semistable nilpotent Higgs bundles of small ranks over the closed fiber with trivial Chern classes; in Section 7, we prove a rigidity theorem for Fontaine modules whose associated graded Higgs modules are mod $p$ stable; in Appendix A, we (joint with Y.-H. Yang) prove that a semistable Higgs module of small rank is strongly semistable, verifying partially a conjecture in the first version of the paper [14].

\section{Preliminaries on Fontaine modules}

The category of Fontaine modules, as introduced by G. Fatlings in [4], originates from number theory. In the seminal paper [7], Fontaine and Laffaille introduced the category $M F^{f, q}$ (resp. $M F_{\text {tor }}^{f, q}$ ) of strongly divisible filtered modules over $W$ (resp. of finite length) and constructed an exact and faithful contravariant functor from the previous category to the category of representations of the Galois group of the local field $K$ (resp. of finite length). A representation lying in the image of the functor is said to be crystalline. The significance of the category, as shown in another seminal paper [8] by Fontaine-Messing, is due to the fact that the crystalline cohomologies of many proper algebraic varieties over $W$ lie in the category. In the above cited paper, Faltings generalized both results to a geometric base (see also [5] for the generalization to the semistable reduction case and the case of a very ramified base ring). For us, this category plays the role connecting a certain category of $p$-adic Higgs modules with a cartain category of 
$p$-adic representations of algebraic fundamental groups. From the point of view of nonabelian Hodge theory, this category is a nice $p$-adic analogue of polarized complex variations of Hodge structure, a special but important class of the socalled harmonic bundles. One of principal aims of this paper is to establish a correspondence between the category of Fontaine modules and the category of one-periodic Higgs-de Rham flows, in both positive and mixed characteristics. This section is devoted to a brief exposition of this category and related known results.

Remark 2.1. We shall remind the reader of the category of $F$-T-crystals developed in the monograph [24], which is also a p-adic analogue of the category of complex variations of Hodge structure (with no emphasis on polarization). This category is intimately related to the category of Fontaine modules (see particularly [24, Proposition 5.3.9]). It is interesting to relate it to a certain category of Higgs modules. This task has not been touched upon in this paper.

For clarity, we start with the $p$-torsion free Fontaine modules. Our exposition is based on Ch. II [4] and $\S 3$ [5]. Let $X$ be a smooth $W$-scheme. For an affine subset $U$ flat over $W$, there exists a (nonunique) absolute Frobenius lifting $F_{\hat{U}}$ on its $p$-adic completion $\hat{U}$. An object in the category $M F_{[0, w]}^{\nabla}(\hat{U})$ is a quadruple $\left(H, \nabla, F i l, \Phi_{F_{\hat{U}}}\right)$, where

i) $(H, F i l)$ is a filtered free $\mathcal{O}_{\hat{U}^{-}}$-module with a basis $e_{i}$ of $F i l^{i}, 0 \leq i \leq w$.

ii) $\nabla$ is an integrable connection on $H$ satisfying the Griffiths' transversality:

$$
\nabla\left(F i l^{i}\right) \subset F i l^{i-1} \otimes \Omega_{\hat{U}} .
$$

iii) The relative Frobenius is an $\mathcal{O}_{\hat{U}}$-linear morphism $\Phi_{F_{\hat{U}}}: F_{\hat{U}}^{*} H \rightarrow H$ with the strong $p$-divisible property: $\Phi_{F_{\hat{U}}}\left(F_{\hat{U}}^{*} F i l^{i}\right) \subset p^{i} H$ and

$$
\sum_{i=0}^{w} \frac{\Phi_{F_{\hat{U}}}\left(F_{\hat{U}}^{*} F i l^{i}\right)}{p^{i}}=H .
$$

iv) The relative Frobenius $\Phi_{F_{\hat{U}}}$ is horizontal with respect to the connection $F_{\hat{U}}^{*} \nabla$ on $F_{\hat{U}}^{*} H$ and $\nabla$ on $H$.

The filtered-freeness in i) means that the filtration $F i l$ on $H$ has a splitting such that each $F i l^{i}$ is a direct sum of several copies of $\mathcal{O}_{\hat{U}}$. Equivalently, Fil is a finite exhaustive decreasing filtration of free $\mathcal{O}_{\hat{U}}$-submodules which is split. The pull-back connection $F_{\hat{U}}^{*} \nabla$ on $F_{\hat{U}}^{*} H$ is the connection defined by the formula

$$
F_{\hat{U}}^{*} \nabla(f \otimes e)=d f \otimes e+f \cdot\left(d F_{\hat{U}} \otimes 1\right)(1 \otimes \nabla(e)), f \in \mathcal{O}_{\hat{U}},\left.e \in H\right|_{\hat{U}} .
$$

The horizontal condition iv) is expressed by the commutativity of the diagram

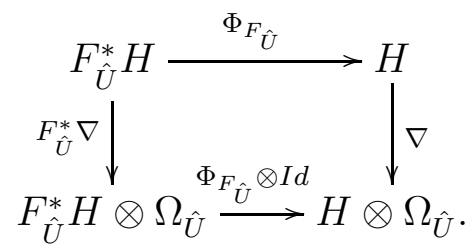


As there is no canonical Frobenius liftings on $\hat{U}$, one must know how the relative Frobenius changes under another Frobenius lifting. This is expressed by a Taylor formula. Let $\hat{U}=\operatorname{Spf} R$ and $F: R \rightarrow R$ an absolute Frobenius lifting. Choose a system of étale local coordinates $\left\{t_{1}, \cdots, t_{d}\right\}$ of $U$ (namely fix an étale map $\left.U \rightarrow \operatorname{Spec}\left(W\left[t_{1}, \cdots, t_{d}\right]\right)\right)$. Let $R^{\prime}$ be any $p$-adically complete, $p$-torsion free $W$-algebra, equipped with a Frobenius lifting $F^{\prime}: R^{\prime} \rightarrow R^{\prime}$ and a morphism of $W$-algebras $\iota: R \rightarrow R^{\prime}$. Then the relative Frobenius $\Phi_{F^{\prime}}: F^{* *}\left(\iota^{*} H\right) \rightarrow \iota^{*} H$ is the composite

$$
F^{*} \iota^{*} H \stackrel{\alpha}{\cong} \iota^{*} F^{*} H \stackrel{\iota^{*} \Phi_{F}}{\longrightarrow} \iota^{*} H,
$$

where the isomorphism $\alpha$ is given by the formula:

$$
\alpha(e \otimes 1)=\sum_{\underline{i}} \nabla \frac{i}{\partial}(e) \otimes \frac{z^{\underline{i}}}{\underline{i} !} .
$$

Here $\underline{i}=\left(i_{1}, \cdots, i_{d}\right)$ is a multi-index, and $z^{\underline{i}}=z_{1}^{i_{1}} \cdots z_{d}^{i_{d}}$ with $z_{i}=F^{\prime} \circ \iota\left(t_{i}\right)-\iota \circ$ $F\left(t_{i}\right), 1 \leq i \leq d$, and $\nabla_{\frac{\partial}{\partial}}^{j}=\nabla_{\partial_{t_{1}}}^{i_{1}} \cdots \nabla_{\partial_{t_{d}}}^{i_{d}}$.

Let $\mathcal{U}=\left\{U_{i}\right\}_{i \in I}$ be an open affine covering of $X$ over $W$. For each $i$, let $F_{\hat{U}_{i}}$ be an absolute Frobenius lifting over $\hat{U}_{i}$. Then, after [4, Theorem 2.3], when $w \leq p-1$, the local categories $\left\{M F_{[0, w]}^{\nabla}\left(\hat{U}_{i}\right)\right\}_{i \in I}$ glue into the category $M F_{[0, w]}^{\nabla}(X)$. Its object is called a Fontaine module over $X / W$, and will be denoted again by a quadruple $(H, \nabla, F i l, \Phi)$, although the relative Frobenius $\Phi$ is only locally defined and depends on a choice of absolute Frobenius lifting locally. For an open affine $U$ together with an absolute Frobenius lifting $F_{\hat{U}}$ over $\hat{U}$, the symbol $\Phi_{\left(U, F_{\hat{U}}\right)}$ means the evaluation of $\Phi$ at $\left(U, F_{\hat{U}}\right)$.

Example 2.2. Let $f: Y \rightarrow X$ be a proper smooth morphism over $W$ of relative dimension $w \leq p-2$ between smooth $W$-schemes. Assume that the relative Hodge cohomologies $R^{i} f_{*} \Omega_{Y}^{j}, i+j=w$ have no torsion. By Theorem 6.2 [4], the crystalline direct image $R^{w} f_{*}\left(\mathcal{O}_{Y}, d\right)$ is an object in $M F_{[0, w]}^{\nabla}(X / W)$.

The fundamental theorem of Fontaine-Laffaille (see [7, Theorem 3.3] for $X=$ Spec $W$ ) and Faltings (see [4, Theorem $\left.2.6^{*}\right]$ ), which is a $p$-adic analogue of the Riemann-Hilbert correspondence over $\mathbb{C}$, reads:

Theorem 2.3 (Fontaine-Laffaille-Faltings correspondence). Notation as above. Assume furthermore $X$ is proper over $W$ and $w \leq p-2$. There exists a fully faithful contravariant functor $\mathbf{D}$ from the category $M F_{[0, w]}^{\nabla}(X / W)$ to the category of étale local systems over $X_{K}$.

The image of the functor $\mathbf{D}$ is called crystalline sheaves of Hodge-Tate weight $n$ over $X_{K}$. We remind the reader that the functor $\mathbf{D}$ in [4] is covariant and its image is the category of dual crystalline sheaves. In the above theorem for torsion free Fontaine modules as well as its $p$-torsion analogue below, we actually use the dual of the functor $\mathbf{D}$. 
Variant 1: p-torsion. A p-torsion Fontaine module is formulated in a similar way. In fact, the previous category $M F_{[0, w]}^{\nabla}(X / W)$ is the $p$-adic limit of its torsion variant (see c)-d). Ch. II [4]). The major modification in the $p$-torsion case occurs to the formulation of strong $p$-divisibility. Note that Equation (2.1.1) does not make sense in the $p$-torsion case. For each natural number $n$, a strict $p^{n}$-torsion Fontaine module $(H, \nabla, F i l, \Phi)$ means the following: $H$ is a finitely generated $\mathcal{O}_{X_{n}}$-module; $F i l$ is a finite exhaustive decreasing filtration of $\mathcal{O}_{X_{n}}{ }^{-}$ submodules on $H$ satisfying Griffiths' transversality with respect to an integrable connection $\nabla ; \Phi$ is strongly $p$-divisible, namely, the evaluation of $\Phi$ at $\left(U, F_{\hat{U}}\right)$ is an $\mathcal{O}_{U_{n}}$-isomorphism $\left(U_{n}:=U \otimes \mathbb{Z} / p^{n} \mathbb{Z}, F_{U_{n}}:=F_{\hat{U}} \otimes \mathbb{Z} / p^{n} \mathbb{Z}\right)$ :

$$
\Phi_{\left(U_{n}, F_{U_{n}}\right)}:\left.\left.F_{U_{n}}^{*} \tilde{H}\right|_{U_{n}} \cong H\right|_{U_{n}}
$$

where $\tilde{H}$ is the quotient $\oplus_{i=0}^{w} F i l^{i} / \sim$ with $x \sim p y$ for any $x \in F i l^{i}$ and $y$ the image of $x$ under the natural inclusion $F i l^{i} \hookrightarrow F i l^{i-1}$; the horizontal property for $\Phi$ is formulated as in the non-torsion case, which means explicitly the following commutative diagram:

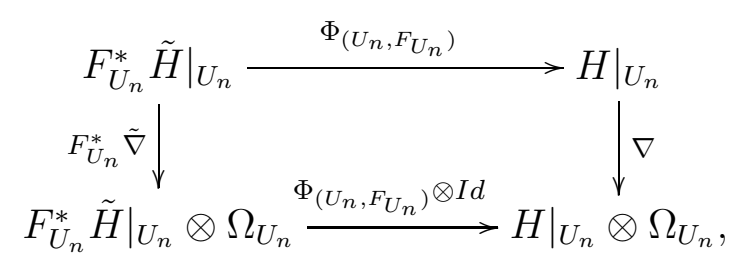

where $\tilde{\nabla}$ is the integral $p$-connection (see Definition 4.4) over $\tilde{H}$ induced by $\nabla$ and $F_{U_{n}}^{*} \tilde{\nabla}$ is similarly defined as $F_{\hat{U}}^{*} \nabla$ in the non $p$-torsion case by replacing $\nabla$ resp. $d F_{\hat{U}}$ in the composite therein with $\tilde{\nabla}$ resp. $\frac{d F_{U_{n}}}{p}$ (see Formula (4.15.1) for a local expression). Let $M F_{[0, w], n}^{\nabla}(X / W)$ denote the category of strict $p^{m}$-torsion Fontaine modules. The Fontaine-Laffaille-Faltings correspondence as given above is achieved by taking the $p$-adic limit of its $p$-torsion analogue.

Remark 2.4. Using Fitting ideals, Faltings [4, Theorem 2.1] shows that $(H, F i l)$ is indeed locally filtered free. A slight generalization obtained by A. Ogus using a different method is given in [24, Theorem 5.3.3]. Note also that, in the formulation of the category $M F_{[0, w], n}^{\nabla}(X / W)$, one actually requires only the existence of a model $X_{n+1}$ over $W_{n+1}$. Although objects of this category are defined over $X_{n}$, the horizontality of the relative Frobenius uses the operator $\frac{d F_{U_{n+1}}}{p}$, where $F_{U_{n+1}}$ is an absolute Frobenius lifting on an open affine $U_{n+1} \subset X_{n+1}$. Also, the existence of $X_{n+1}$ is required for the sake of the transition of two evaluations of the relative Frobenius via the Taylor formula. Therefore, this category requires only the existence of a smooth $W_{n+1}$-scheme $X_{n+1}$. In this case, we shall denote it by $M F_{[0, w]}^{\nabla}\left(X_{n+1} / W_{n+1}\right)$.

Variant 2: extra endomorphism. For our purpose, we need to introduce the category $M F_{[0, w], f}^{\nabla}(X / W)$ of Fontaine modules with endomorphism structure $W\left(\mathbb{F}_{p^{f}}\right)$ for each $f \in \mathbb{N}$. It consists of five tuples $(H, \nabla, F i l, \Phi, \iota)$, where $(H, \nabla, F i l, \Phi)$ is 
a torsion-free Fontaine module and

$$
\iota: W\left(\mathbb{F}_{p^{f}}\right) \hookrightarrow \operatorname{End}_{M F}(H, \nabla, F i l, \Phi)
$$

is an embedding of $\mathbb{Z}_{p}$-algebras. A morphism of this category is a morphism in $M F_{[0, w]}^{\nabla}(X / W)$ respecting the endomorphism structure $\iota$. Clearly, the category for $f=1$ is nothing but the category of Fontaine modules. We introduce similarly its $p$-torsion counterpart $M F_{[0, w], n, f}^{\nabla}(X / W)$ (and $\left.M F_{[0, w], f}^{\nabla}\left(X_{n+1} / W_{n+1}\right)\right)$, where the extra endomorphism structure is given by an embedding of $\mathbb{Z} / p^{n} \mathbb{Z}$-algebras:

$$
\iota: W_{n}\left(\mathbb{F}_{p^{f}}\right) \hookrightarrow \operatorname{End}_{M F}(H, \nabla, F i l, \Phi) .
$$

\section{Higgs CORRESPONDENCE IN POSITIVE CHARACTERISTIC}

Let us begin with the definitions of a (pre)periodic Higgs-de Rham flow and a (pre)periodic Higgs module. Let $X_{1}$ be a smooth variety over $k$ and $X_{2}$ a $W_{2^{-}}$ lifting of $X_{1}$.

Definition 3.1. A preperiodic Higgs-de Rham flow over $X_{1}$ (with respect to the given $W_{2}$-lifting $\left.X_{2}\right)$ is a tuple $\left(E, \theta, F i l_{0}, \cdots, F i l_{e+f-1}, \phi\right)$, where $e \geq 0$ and $f \geq 1$ are two integers, $(E, \theta)$ is a Higgs module in the category $H I G_{p-1}\left(X_{1}\right)$, $F i l_{i}, 0 \leq i \leq e+f-1$ is a Griffiths transverse filtration on $C_{1}^{-1}\left(E_{i}, \theta_{i}\right)$ where $\left(E_{0}, \theta_{0}\right)=(E, \theta)$ and

$$
\left(E_{i}, \theta_{i}\right):=G r_{F i l_{i-1}}\left(H_{i-1}, \nabla_{i-1}\right), 1 \leq i \leq e+f
$$

is inductively defined, and $\phi$ is an isomorphism of graded Higgs modules

$$
\left(E_{e+f}, \theta_{e+f}\right) \cong\left(E_{e}, \theta_{e}\right) .
$$

It is said to be periodic of period $f$ (or $f$-periodic) if the integer $e$ in above is zero. The $\left(E_{i}, \theta_{i}\right) \mathrm{s}$ (resp. $\left.\left(H_{i}, \nabla_{i}\right) \mathrm{s}\right)$ are called the Higgs (resp. de-Rham) terms of the flow. A Higgs module $(E, \theta)$ over $X_{1}$ is said to be (pre)periodic if there exists a (pre)periodic Higgs-de Rham flow with the leading Higgs term $(E, \theta)$.

One may complete a preperiodic Higgs-de Rham flow over $X_{1}$ into a Higgs-de Rham flow in a natural way: note that the isomorphism $\phi$ induces the isomorphism

$$
C_{1}^{-1}(\phi): C_{1}^{-1}\left(E_{e+f}, \theta_{e+f}\right) \cong C_{1}^{-1}\left(E_{e}, \theta_{e}\right),
$$

and therefore one obtains naturally the filtration $F i l_{e+f}$ on $C_{1}^{-1}\left(E_{e+f}, \theta_{e+f}\right)$ by pulling back $F i l_{e}$ via the isomorphism. Then continue successively.

This section aims to establish the Higgs correspondence between the category of periodic Higgs-de Rham flows over $X_{1}$ and the category of strict $p$-torsion Fontaine modules with extra endomorphism structure. Let us introduce the category $H D F_{w, f}\left(X_{2} / W_{2}\right)$ as follows : its object is given by a periodic Higgs-de Rham flow $\left(E, \theta, F i l_{0}, F i l_{1}, \cdots, F i l_{f-1}, \phi\right)$ over $X_{1}$ such that each filtration $F i l_{i}$ is of level $\leq w$. Note that $(E, \theta)$ in a periodic Higgs-de Rham must be a graded Higgs module. A morphism between two objects is a morphism of graded Higgs modules respecting the additional structures. As an illustration, we explain a morphism in the category $H D F_{w, 1}\left(X_{2} / W_{2}\right)$ of one-periodic Higgs-de Rham flow 
in detail. Let $\left(E_{i}, \theta_{i}, F i l_{i}, \phi_{i}\right), i=1,2$ be two objects in the category. Then a morphism

$$
f:\left(E_{1}, \theta_{1}, F i l_{1}, \phi_{1}\right) \rightarrow\left(E_{2}, \theta_{2}, F i l_{2}, \phi_{2}\right)
$$

is given by a morphism of graded Higgs modules

$$
f:\left(E_{1}, \theta_{1}\right) \rightarrow\left(E_{2}, \theta_{2}\right)
$$

such that the induced morphism of flat modules (by the functoriality of $C_{1}^{-1}$ )

$$
C_{1}^{-1}(f): C_{1}^{-1}\left(E_{1}, \theta_{1}\right) \rightarrow C_{1}^{-1}\left(E_{2}, \theta_{2}\right)
$$

preserves the filtrations, and moreover the induced morphism of graded Higgs modules is compatible with $\phi \mathrm{s}$, that is, the following diagram of natural morphisms commutes:

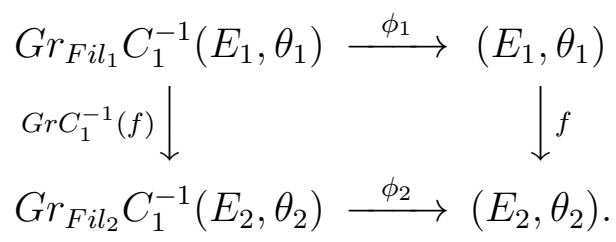

Recall that $M F_{[0, w], f}^{\nabla}\left(X_{2} / W_{2}\right)$ is the category of strict $p$-torsion Fontaine modules with extra endomorphism $\mathbb{F}_{p^{f}}$. The Higgs correspondence in positive characteristic is the following

Theorem 3.2. Notation as above. Let $w \leq p-1$ and $f$ be a natural number. Then there is an equivalence of categories between the category $M F_{[0, w], f}^{\nabla}\left(X_{2} / W_{2}\right)$ and the category $\mathrm{HDF}_{w, f}\left(X_{2} / W_{2}\right)$.

We take an open covering $\left\{U_{i}\right\}$ of $X_{2} / W_{2}$ consisting of open affine subsets which are smooth over $W_{2}$, together with an absolute Frobenius lifting $F_{U_{i}}$ on each $U_{i}$. By modulo $p$, one obtains an open affine covering $\left\{U_{i, 1}\right\}$ for $X_{1}$. We show first a special case of the theorem, namely the $f=1$ case.

Proposition 3.3. There is an equivalence of categories between the category of strict p-torsion Fontaine modules and the category of one-periodic Higgs-de Rham flows over $X_{1}$.

For simplicity, we denote $M F$ for $M F_{[0, w], 1}^{\nabla}\left(X_{2} / W_{2}\right)$ and $H D F$ for $H D F_{w, 1}\left(X_{2} / W_{2}\right)$. In the following paragraph, we shall construct two functors

$$
\mathcal{G R}: M F \rightarrow H D F, \quad \mathcal{I C}: H D F \rightarrow M F,
$$

and then show they are quasi-inverse to each other. For an $(H, \nabla, F i l, \Phi) \in M F$, let $(E, \theta):=G r_{F i l}(H, \nabla)$ be the associated graded Higgs module. The following lemma gives the first functor.

Lemma 3.4. There is a filtration Fil $l_{\exp }$ on $C_{1}^{-1}(E, \theta)$ together with an isomorphism of graded Higgs modules

$$
\phi_{\text {exp }}: G r_{F i l_{\text {exp }}}\left(C_{1}^{-1}(E, \theta)\right) \cong(E, \theta),
$$

which is induced by the filtration Fil and the relative Frobenius $\Phi$. 
Proof. By [13, Proposition 1.4], the relative Frobenius induces an isomorphism of flat modules

$$
\tilde{\Phi}: C_{1}^{-1}(E, \theta) \cong(H, \nabla) .
$$

So we define $F i l_{\text {exp }}$ on $C_{1}^{-1}(E, \theta)$ to be the inverse image of $F i l$ on $H$ by $\tilde{\Phi}$. It induces tautologically an isomorphism of graded Higgs modules

$$
\phi_{\exp }=\operatorname{Gr}(\tilde{\Phi}): G r_{F i l_{\text {exp }}}\left(C_{1}^{-1}(E, \theta)\right) \cong(E, \theta) .
$$

Next, the functor $C_{1}^{-1}$ induces the second functor $\mathcal{I C}$ as follows. Given an object $(E, \theta, F i l, \phi) \in H D F$, we define the triple by

$$
(H, \nabla, F i l)=\left(C_{1}^{-1}(E, \theta), F i l\right) .
$$

What remains is to produce a relative Frobenius $\Phi$ from the $\phi$. This is the most technical point of the whole proof. Following Faltings [4, Ch. II. d)], it suffices to give for each pair $\left(U_{i}, F_{U_{i}}\right)$ an $\mathcal{O}_{U_{i, 1}}$-morphism

$$
\Phi_{\left(U_{i}, F_{U_{i}}\right)}:\left.\left.F_{U_{i, 1}}^{*} G r_{F i l} H\right|_{U_{i, 1}} \rightarrow H\right|_{U_{i, 1}} \text {, }
$$

where $F_{U_{i, 1}}$ is the absolute Frobenius of $U_{i, 1}$, satisfying the following conditions:

(1) strong $p$-divisibility, that is, $\Phi_{\left(U_{i}, F_{U_{i}}\right)}$ is an isomorphism,

(2) horizontal property,

(3) over each $U_{i j, 1}:=U_{i, 1} \cap U_{j, 1}, \Phi_{\left(U_{i}, F_{U_{i}}\right)}$ and $\Phi_{\left(U_{j}, F_{U_{j}}\right)}$ are related via the Taylor formula. Precisely, if the gluing map for $H_{i}:=\left.H\right|_{U_{i, 1}}$ and $H_{j}:=$ $\left.H\right|_{U_{j, 1}}$ is $G_{i j}$, then we shall have the following commutative diagram:

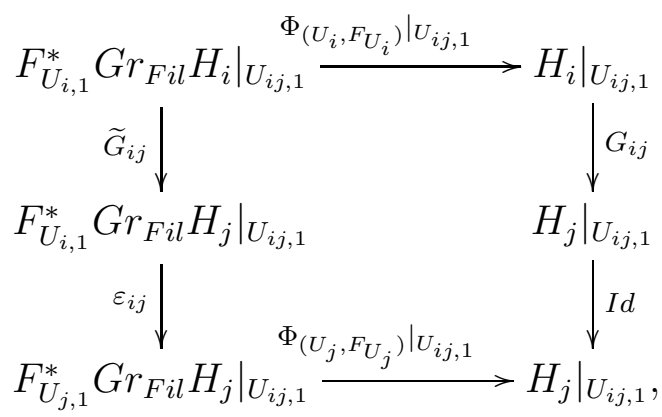

where $\widetilde{G}_{i j}$ denotes the obvious map induced by $G_{i j}$, and $\varepsilon_{i j}$ is defined by the Taylor formula which is given by the following expression:

$$
e \otimes 1 \rightarrow e \otimes 1+\sum_{|\underline{k}|=1}^{w}\left(\theta_{\partial}^{\prime}\right)^{\underline{k}}(e) \otimes \frac{z^{\underline{k}}}{p^{\mid \underline{k}} \underline{k} !},
$$

where $\theta^{\prime}$ denotes the Higgs field of $G r_{F i l}\left(H_{j}, \nabla\right)$. Here we take a system of étale local coordinates $\left\{\tilde{t}_{1}, \cdots, \tilde{t}_{d}\right\}$ of $U_{i j, 2}$, which induces a system of étale local coordinates $\left\{t_{1}, \cdots, t_{d}\right\}$ on $U_{i j, 1}$, and $\underline{k}=\left(k_{1}, \cdots, k_{d}\right)$ is a multi-index, $z^{\underline{k}}=z_{1}^{k_{1}} \cdots z_{d}^{k_{d}}$ with

$$
z_{k}=F_{U_{i, 2}}^{*}\left(\tilde{t}_{k}\right)-F_{U_{j, 2}}^{*}\left(\tilde{t}_{k}\right)
$$

and $\left(\theta^{\prime}\right) \frac{k}{\partial}=\left(\theta_{\partial_{t_{1}}}^{\prime}\right)^{k_{1}} \cdots\left(\theta_{\partial_{t_{d}}}^{\prime}\right)^{k_{d}}$. 
Recall that $H=\left\{H_{i}:=\left.F_{U_{i, 1}}^{*} E\right|_{U_{i, 1}}, G_{i j}\right\}_{i \in I}$, where $G_{i j}$ has similar expression of $\varepsilon_{i j}$ as 3.4.1 (see [13, The poof of Proposition 1.4]) :

$$
G_{i j}(e \otimes 1)=e \otimes 1+\sum_{|\underline{k}|=1}^{w} \theta \frac{k}{\partial}(e) \otimes \frac{z^{\underline{k}}}{p^{|\underline{k}|} \underline{k} !} .
$$

We define

$$
\Phi_{\left(U_{i}, F_{U_{i}}\right)}=F_{U_{i, 1}}^{*} \phi:\left.\left.F_{U_{i, 1}}^{*} G r_{F i l} H\right|_{U_{i, 1}} \rightarrow F_{U_{i, 1}}^{*} E\right|_{U_{i, 1}} .
$$

By construction, $\Phi_{\left(U_{i}, F_{U_{i}}\right)}$ is strongly $p$-divisible (this is Condition (1)). As $\phi$ is globally defined, we have the following diagram:

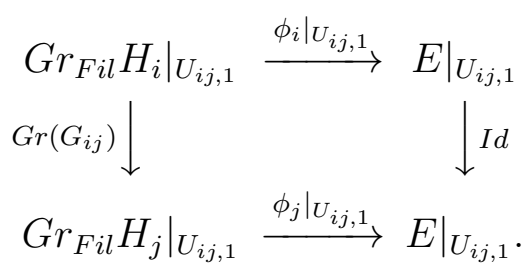

Pulling back the above diagram via $F_{U_{i, 1}}^{*}$, we get the following diagram:

$$
\begin{array}{ccc}
\left.\left.F_{U_{i, 1}}^{*} G r_{F i l} H_{i}\right|_{U_{i j, 1}} \stackrel{\left.F_{U_{i, 1}}^{*}\left(\phi_{i}\right)\right|_{U_{i j, 1}}}{\longrightarrow} F_{U_{i, 1}}^{*} E\right|_{U_{i j, 1}} \\
\widetilde{G_{i j}} \downarrow & \downarrow I d \\
\left.\left.F_{U_{i, 1}}^{*} G r_{F i l} H_{j}\right|_{U_{i j, 1}} \stackrel{\left.F_{U_{i, 1}}^{*}\left(\phi_{j}\right)\right|_{U_{i j, 1}}}{\longrightarrow} F_{U_{i, 1}}^{*} E\right|_{U_{i j, 1}} .
\end{array}
$$

Then we extend it to the following diagram:

$$
\begin{array}{ccc}
\left.\left.F_{U_{i, 1}}^{*} G r_{F i l} H_{i}\right|_{U_{i j, 1}} \stackrel{\left.F_{U_{i, 1}}^{*}\left(\phi_{i}\right)\right|_{U_{i j, 1}}}{\longrightarrow} F_{U_{i, 1}}^{*} E\right|_{U_{i j, 1}} \\
F_{U_{i j}} \downarrow & \left.\left.\downarrow r_{F i l} H_{j}\right|_{U_{i j, 1}} \stackrel{\left.F_{U_{i, 1}}^{*}\left(\phi_{j}\right)\right|_{U_{i j, 1}}}{\longrightarrow} F_{U_{i, 1}}^{*} E\right|_{U_{i j, 1}} \\
\varepsilon_{i j} \downarrow & \downarrow G_{i j} \\
\left.\left.F_{U_{j, 1}}^{*} G r_{F i l} H_{j}\right|_{U_{i j, 1}} \stackrel{\left.F_{U_{j, 1}}^{*}\left(\phi_{j}\right)\right|_{U_{i j, 1}}}{\longrightarrow} F_{U_{j, 1}}^{*} E\right|_{U_{i j, 1}}
\end{array}
$$

As $\phi \circ \theta^{\prime}=\theta \circ \phi$, we have for any local section $e$ of $\left.G r_{F i l} H_{j}\right|_{U_{i j, 1}}$,

$$
\begin{aligned}
G_{i j} \circ F_{U_{i, 1}}^{*}\left(\phi_{j}\right)(e \otimes 1) & =\phi_{j}(e) \otimes 1+\sum_{|\underline{k}|=1}^{w} \theta \frac{k}{\partial}\left(\phi_{j}(e)\right) \otimes \frac{z^{\underline{k}}}{p^{|\underline{k}|} \underline{k} !} \\
& =\phi_{j}(e) \otimes 1+\sum_{|\underline{k}|=1}^{w} \phi_{j}\left(\left(\theta^{\prime}\right) \frac{k}{\partial}(e)\right) \otimes \frac{z^{\underline{k}}}{p^{|\underline{k}|} \underline{k} !} \\
& =F_{U_{j, 1}}^{*}\left(\phi_{j}\right) \circ \varepsilon_{i j}(e \otimes 1) .
\end{aligned}
$$

So the lower square of the last diagram is commutative (this is Condition (3)). What remains to show is Condition (2).

Lemma 3.5. Each $\Phi_{\left(U_{i}, F_{U_{i}}\right)}$ is horizontal with respect to $\nabla$. 
Proof. Put $\tilde{H}=G r_{F i l} H, \theta^{\prime}=G r_{F i l} \nabla, \Phi_{i}=\Phi_{\left(U_{i}, F_{U_{i}}\right)}$ and $F_{U_{i, 1}}$ the absolute Frobenius over $U_{i, 1}$. Following Faltings [4, Ch. II.. d)], it suffices to show the commutativity of the following diagram

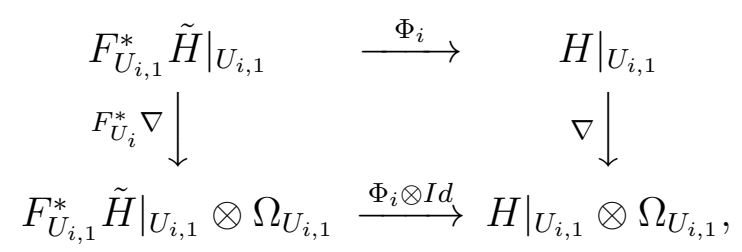

where $F_{U_{i}}^{*} \nabla$ is a connection induced by $\frac{d F_{U_{i}}}{p}\left(F_{U_{i, 1}}^{*} \theta^{\prime}\right)$, i.e. the composite of

$$
\left.\left.\left.F_{U_{i, 1}}^{*} \tilde{H}\right|_{U_{i, 1}} \stackrel{F_{U_{i, 1}}^{*}\left(\theta^{\prime}\right)}{\longrightarrow} F_{U_{i, 1}}^{*} \tilde{H}\right|_{U_{i, 1}} \otimes F_{U_{i, 1}}^{*} \Omega_{U_{i, 1}} \stackrel{I d \otimes \frac{d F_{U_{i}}}{\longrightarrow}}{\longrightarrow} F_{U_{i, 1}}^{*} \tilde{H}\right|_{U_{i, 1}} \otimes \Omega_{U_{i, 1}} .
$$

Thus it is to show the commutativity of the next diagram:

$$
\begin{aligned}
& \left.\left.F_{U_{i, 1}}^{*} \tilde{H}\right|_{U_{i, 1}} \stackrel{F_{U_{i, 1}}^{*}(\phi)}{\longrightarrow} \quad F_{U_{i, 1}}^{*} E\right|_{U_{i, 1}} \\
& \frac{d F_{U_{i}}}{p}\left(F_{U_{i, 1}}^{*}\left(\theta^{\prime}\right)\right) \downarrow \quad \frac{d F_{U_{i}}}{p}\left(F_{U_{i, 1}}^{*}(\theta)\right) \downarrow \\
& \left.\left.F_{U_{i, 1}}^{*} \tilde{H}\right|_{U_{i, 1}} \otimes \Omega_{U_{i, 1}} \stackrel{F_{U_{i, 1}}^{*}(\phi) \otimes I d}{\longrightarrow} F_{U_{i, 1}}^{*} E\right|_{U_{i, 1}} \otimes \Omega_{U_{i, 1}} .
\end{aligned}
$$

As $\phi$ is a morphism of graded Higgs modules, one has the following commutative diagram:

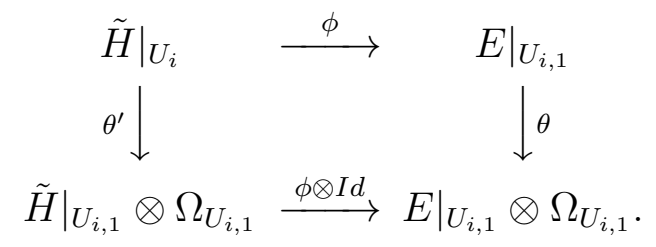

The pullback via $F_{U_{i, 1}}^{*}$ of the above diagram yields commutative diagrams:

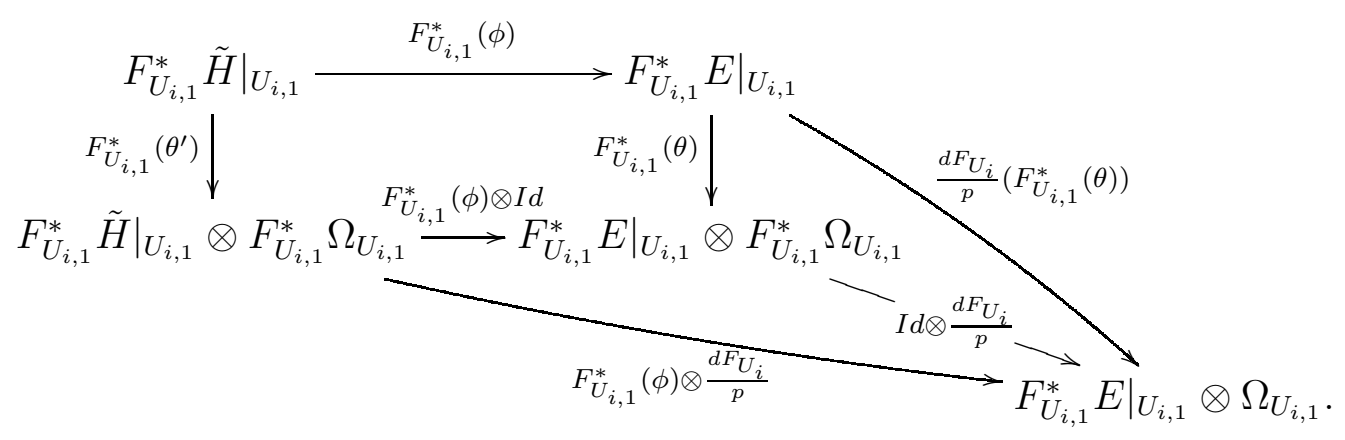

Chasing the outside of the above diagram gives the required commutativity. Now we can prove Proposition 3.3,

Proof. The equivalence of categories follows by providing natural isomorphisms of functors:

$$
\mathcal{G R} \circ \mathcal{I C} \cong I d, \quad \mathcal{I C} \circ \mathcal{G R} \cong I d
$$


We define first a natural isomorphism $\mathcal{A}$ from $\mathcal{I C} \circ \mathcal{G R}$ to $I d$ : for $(H, \nabla, F i l, \Phi) \in$ $M F$, put

$$
(E, \theta, F i l, \phi)=\mathcal{G} \mathcal{R}(H, \nabla, F i l, \Phi), \quad\left(H^{\prime}, \nabla^{\prime}, F i l^{\prime}, \Phi^{\prime}\right)=\mathcal{I C}(E, \theta, F i l, \phi) .
$$

Then one verifies that the map

$$
\tilde{\Phi}:\left(H^{\prime}, \nabla^{\prime}\right)=C_{1}^{-1} \circ G r_{F i l}(H, \nabla) \cong(H, \nabla)
$$

gives an isomorphism from $\left(H^{\prime}, \nabla^{\prime}, F i l^{\prime}, \Phi^{\prime}\right)$ to $(H, \nabla, F i l, \Phi)$ in the category $M F$. We call it $\mathcal{A}(H, \nabla, F i l, \Phi)$. It is straightforward to verify that $\mathcal{A}$ is indeed a transformation. Conversely, a natural isomorphism $\mathcal{B}$ from $\mathcal{G R} \circ \mathcal{I C}$ to $I d$ is given as follows: for $(E, \theta, F i l, \phi)$, put

$$
(H, \nabla, F i l, \Phi)=\mathcal{I C}(E, \theta, F i l, \phi) \quad\left(E^{\prime}, \theta^{\prime}, F i l^{\prime}, \phi^{\prime}\right)=\mathcal{G} \mathcal{R}(H, \nabla, F i l, \Phi) .
$$

Then $\phi: G r_{F i l} \circ C_{1}^{-1}(E, \theta) \cong(E, \theta)$ induces an isomorphism from $\left(E^{\prime}, \theta^{\prime}, F i l^{\prime}, \phi^{\prime}\right)$ to $(E, \theta, F i l, \phi)$ in $H D F$, which we define to be $\mathcal{B}(E, \theta, F i l, \phi)$. It is direct to check that $\mathcal{B}$ is a natural isomorphism.

Before moving to the proof of Theorem 3.2 in general, we shall introduce an intermediate category, the category of one-periodic Higgs-de Rham flows with endomorphism structure $\mathbb{F}_{p^{f}}$ : an object is a five tuple $(E, \theta, F i l, \phi, \iota)$, where $(E, \theta, F i l, \phi)$ is object in $H D F$ and $\iota: \mathbb{F}_{p^{f}} \hookrightarrow \operatorname{End}_{H D F}(E, \theta, F i l, \phi)$ is an embedding of $\mathbb{F}_{p}$-algebras. As an immediate consequence of Proposition 3.3, we have

Corollary 3.6. There is an equivalence of categories between the category of strict p-torsion Fontaine modules with endomorphism structure $\mathbb{F}_{p^{f}}$ and the category of one-periodic Higgs-de Rham flows over $X_{1}$ with endomorphism structure $\mathbb{F}_{p^{f}}$.

Obviously, Corollary 3.6 and the next proposition will complete the proof of Theorem 3.2.

Proposition 3.7. There is an equivalence of categories between the category of one-periodic Higgs-de Rham flows of level $\leq w$ over $X_{1}$ with endomorphism structure $\mathbb{F}_{p^{f}}$ and the category $H D F_{w, f}\left(X_{2} / W_{2}\right)$.

Start off with an object $\left(E, \theta, F i l_{0}, \cdots, F i l_{f-1}, \phi\right)$ in $H D F_{w, f}\left(X_{2} / W_{2}\right)$. Put

$$
(G, \eta):=\bigoplus_{i=0}^{f-1}\left(E_{i}, \theta_{i}\right)
$$

with $\left(E_{0}, \theta_{0}\right)=(E, \theta)$. As the functor $C_{1}^{-1}$ is compatible with direct sum, one has the identification

$$
C_{1}^{-1}(G, \eta)=\bigoplus_{i=0}^{f-1} C_{1}^{-1}\left(E_{i}, \theta_{i}\right)
$$

We equip $C_{1}^{-1}(G, \eta)$ with the filtration $F i l=\bigoplus_{i=0}^{f-1} F i l_{i}$ by the above identification. Also $\phi$ induces a natural isomorphism of graded Higgs modules

$$
\tilde{\phi}: G r_{F i l} C_{1}^{-1}(G, \eta) \cong(G, \eta)
$$


as follows: as

$$
G r_{F i l} C_{1}^{-1}(G, \eta)=\bigoplus_{i=0}^{r-1} G r_{F i l_{i}} C_{1}^{-1}\left(E_{i}, \theta_{i}\right)
$$

we require that $\tilde{\phi}$ maps the factor $\operatorname{Gr}_{F i l_{i}} C_{1}^{-1}\left(E_{i}, \theta_{i}\right)$ identically to the factor $\left(E_{i+1}, \theta_{i+1}\right)$ for $0 \leq i \leq f-2$ (assume $f \geq 2$ to avoid the trivial case) and the last factor $G r_{F i l_{f-1}}\left(E_{f-1}, \theta_{f-1}\right)$ isomorphically to $\left(E_{0}, \theta_{0}\right)$ via $\phi$. Thus the constructed quadruple $(G, \eta, F i l, \tilde{\phi})$ is a periodic Higgs-de Rham flow of period one.

Lemma 3.8. Notation as above. There is a natural embedding of $\mathbb{F}_{p}$-algebras

$$
\iota: \mathbb{F}_{p^{r}} \rightarrow \operatorname{End}_{H D F}(G, \eta, F i l, \tilde{\phi}) .
$$

Thus the extended tuple $(G, \eta, F i l, \tilde{\phi}, \iota)$ is a one-periodic Higgs-de Rham flow with endomorphism structure $\mathbb{F}_{p^{f}}$.

Proof. Choose a primitive element $\xi_{1}$ in $\mathbb{F}_{p^{f}} \mid \mathbb{F}_{p}$ once and for all. To define the embedding $\iota$, it suffices to specify the image $s:=\iota\left(\xi_{1}\right)$, which is defined as follows: write

$$
(G, \eta)=\left(E_{0}, \theta_{0}\right) \oplus\left(E_{1}, \theta_{1}\right) \oplus \cdots \oplus\left(E_{f-1}, \theta_{f-1}\right) .
$$

Then $s=m_{\xi_{1}} \oplus m_{\xi_{1}^{p}} \oplus \cdots \oplus m_{\xi_{1}^{p f-1}}$, where $m_{\xi_{1}^{p^{i}}}, i=0, \cdots, f-1$ is the multiplication map by $\xi_{1}^{p^{i}}$. It defines an endomorphism of $(G, \eta)$ and preserves Fil on $C_{1}^{-1}(G, \eta)$. Write $\left(G r_{F i l} \circ C_{1}^{-1}\right)(s)$ to be the induced endomorphism of $G r_{F i l} C_{1}^{-1}(G, \eta)$. It remains to verify the commutativity

$$
\tilde{\phi} \circ s=\left(G r_{F i l} \circ C_{1}^{-1}\right)(s) \circ \tilde{\phi} .
$$

In terms of a local basis, it boils down to the following obvious equality

$$
\left(\begin{array}{cccc}
0 & 1 & \ldots & 0 \\
\vdots & \vdots & \ddots & \vdots \\
0 & 0 & \ldots & 1 \\
\phi & 0 & \ldots & 0
\end{array}\right)\left(\begin{array}{cccc}
\xi_{1} & 0 & \ldots & 0 \\
0 & \xi_{1}^{p} & \ldots & 0 \\
\vdots & \vdots & \ddots & \vdots \\
0 & 0 & \ldots & \xi_{1}^{p^{f-1}}
\end{array}\right)=\left(\begin{array}{cccc}
\xi_{1}^{p} & 0 & \ldots & 0 \\
0 & \xi_{1}^{p^{2}} & \ldots & 0 \\
\vdots & \vdots & \ddots & \vdots \\
0 & 0 & \ldots & \xi_{1}
\end{array}\right)\left(\begin{array}{cccc}
0 & 1 & \ldots & 0 \\
\vdots & \vdots & \ddots & \vdots \\
0 & 0 & \ldots & 1 \\
\phi & 0 & \ldots & 0
\end{array}\right) .
$$

Conversely, given a one-periodic Higgs-de Rham flow with endomorphism structure $\mathbb{F}_{p^{f}}$, say $(G, \eta, F i l, \phi, \iota)$, we can associate it an object in $H D F_{w, f}\left(X_{2} / W_{2}\right)$ as follows: the endomorphism $\iota\left(\xi_{1}\right)$ decomposes $(G, \eta)$ into eigenspaces:

$$
(G, \eta)=\bigoplus_{i=0}^{f-1}\left(G_{i}, \eta_{i}\right)
$$

where $\left(G_{i}, \eta_{i}\right)$ is the eigenspace to the eigenvalue $\xi_{1}^{p^{i}}$. The isomorphism $C_{1}^{-1}\left(\iota\left(\xi_{1}\right)\right)$ induces the eigen-decomposition of the de Rham module as well:

$$
\left(C_{1}^{-1}(G, \eta), F i l\right)=\bigoplus_{i=0}^{f-1}\left(C_{1}^{-1}\left(G_{i}, \eta_{i}\right), F i l_{i}\right) .
$$


Under the decomposition, the isomorphism $\phi: G r_{F i l} C_{1}^{-1}(G, \eta) \cong(G, \eta)$ decomposes into $\oplus_{i=0}^{f-1} \phi_{i}$ such that for $i \leq f-2$,

$$
\phi_{i}: G r_{F i i_{i}} C_{1}^{-1}\left(G_{i}, \eta_{i}\right) \cong\left(G_{i+1}, \eta_{i+1}\right),
$$

and $\phi_{f-1}: G r_{F i l_{f-1}} C_{1}^{-1}\left(G_{f-1}, \eta_{f-1}\right) \cong\left(G_{0}, \eta_{0}\right)$. Set $(E, \theta)=\left(G_{0}, \eta_{0}\right)$.

Lemma 3.9. Let $\left(E_{0}, \theta_{0}\right)=(E, \theta)$. Then the filtrations $\left\{F i l_{i}\right\}$ s and isomorphisms of graded Higgs modules $\left\{\phi_{i}\right\}$ s induce inductively the filtration $\widetilde{F i}_{i}$ on $C_{1}^{-1}\left(E_{i}, \theta_{i}\right), i=0, \cdots, f-1$ and the isomorphism of graded Higgs modules

$$
\tilde{\phi}: G r_{\widetilde{\text { Fil }_{f-1}}} C_{1}^{-1}\left(E_{f-1}, \theta_{f-1}\right) \cong(E, \theta) .
$$

Thus the extended tuple $\left(E, \theta, \widetilde{F i} l_{0}, \cdots, \widetilde{F i l}{ }_{f-1}, \tilde{\phi}\right)$ is an object in $H D F_{w, f}\left(X_{2} / W_{2}\right)$.

Proof. The filtration $\widetilde{F i} l_{0}$ on $C_{1}^{-1}\left(E_{0}, \theta_{0}\right)$ is just $F i l_{0}$. Set

$$
\left(E_{1}, \theta_{1}\right)=G r_{F i l_{0}} C_{1}^{-1}\left(E_{0}, \theta_{0}\right) .
$$

Via the isomorphism

$$
C_{1}^{-1}\left(\phi_{0}\right): C_{1}^{-1} G r_{F i l_{0}} C_{1}^{-1}\left(G_{0}, \eta_{0}\right) \cong C_{1}^{-1}\left(G_{1}, \eta_{1}\right)
$$

we obtain the filtration $\widetilde{F i l}_{1}$ on $C_{1}^{-1}\left(E_{1}, \theta_{1}\right)$ from the $F i l_{1}$ on $C_{1}^{-1}\left(G_{1}, \eta_{1}\right)$ by pull-back. By construction, one has the isomorphism

$$
G r C_{1}^{-1}\left(\phi_{0}\right): G r_{\widetilde{F_{i}} l_{1}} C_{1}^{-1}\left(E_{1}, \theta_{1}\right) \cong G r_{F i l_{1}} C_{1}^{-1}\left(G_{1}, \eta_{1}\right)
$$

Repeating the same procedure for $\left(E_{2}, \theta_{2}\right)$ and so on, we shall inductively obtain the filtration $\widetilde{F i} l_{i}$ on $C_{1}^{-1}\left(E_{i}, \theta_{i}\right)$ for $i=1, \cdots, f-1$. Finally we define

$$
\tilde{\phi}: G r_{\widetilde{F i l}} C_{f-1}^{-1}\left(E_{f-1}, \theta_{f-1}\right)=\left(G r_{\widetilde{F i l_{f-1}}} C_{1}^{-1}\right) \circ \cdots \circ\left(G r_{\widetilde{F i l_{0}}} C_{1}^{-1}\right)(E, \theta) \rightarrow(E, \theta)
$$

to be the composite $\left(G r C_{1}^{-1}\right)^{f-1}\left(\phi_{0}\right) \circ \cdots \circ\left(G r C_{1}^{-1}\right)\left(\phi_{f-2}\right) \circ \phi_{f-1}$.

We come to the proof of Proposition 3.7.

Proof. For $f=1$, there is nothing to prove. Suppose $f \geq 2$ in the following. Note that Lemma 3.8 gives a functor $\mathcal{E}$ from $H D F_{w, f}\left(X_{2} / W_{2}\right)$ to the category of one-periodic Higgs-de Rham flows with endomorphism structure $\mathbb{F}_{p^{f}}$, while Lemma 3.9 gives a functor $\mathcal{D}$ in the opposite direction. We show that they give an equivalence of categories. It is direct to see that

$$
\mathcal{D} \circ \mathcal{E}=I d .
$$

So it remains to give a natural isomorphism $\tau$ between $\mathcal{E} \circ \mathcal{D}$ and $I d$. For $(E, \theta, F i l, \phi, \iota)$, let

$$
\mathcal{D}(E, \theta, F i l, \phi, \iota)=\left(G, \eta, F i l_{0}, \cdots, F i l_{f-1}, \tilde{\phi}\right),
$$

and

$$
\mathcal{E}\left(G, \eta, F i l_{0}, \cdots, F i l_{f-1}, \tilde{\phi}\right)=\left(E^{\prime}, \theta^{\prime}, F i l^{\prime}, \phi^{\prime}, \iota^{\prime}\right) .
$$

By the construction, we get that $\left(E^{\prime}, \theta^{\prime}\right)$ is equal to

$$
(G, \eta) \oplus G r_{F i l_{0}} C_{1}^{-1}(G, \eta) \oplus \cdots \oplus\left(G r_{F i l_{f-2}} C_{1}^{-1}\right) \circ \cdots \circ\left(G r_{F i l_{0}} C_{1}^{-1}\right)(G, \eta) .
$$


Let $(E, \theta)=\left(E_{0}, \theta_{0}\right) \oplus\left(E_{1}, \theta_{1}\right) \oplus \cdots \oplus\left(E_{f-1}, \theta_{f-1}\right)$ be the eigen-decomposition of $(E, \theta)$ under $\iota\left(\xi_{1}\right)$. For $1 \leq i \leq f-1$, there is a natural isomorphism $\phi_{i-1} \circ$ $\left(G r C^{-1}\right) \phi_{i-2} \circ \cdots \circ\left(G r C^{-1}\right)^{i-1} \phi_{0}$ of graded Higgs modules between the factors:

$$
\left(G r_{F i l_{i-1}} C_{1}^{-1}\right) \circ\left(G r_{F i l_{i-2}} C_{1}^{-1}\right) \cdots \circ\left(G r_{F i l_{0}} C_{1}^{-1}\right)(G, \eta) \cong\left(E_{i}, \theta_{i}\right) .
$$

Thus $I d \oplus \bigoplus_{i=1}^{f-1} \phi_{i-1} \circ\left(G r C^{-1}\right) \phi_{i-2} \circ \cdots \circ\left(G r C^{-1}\right)^{i-1} \phi_{0}$ provides us an isomorphism of graded Higgs modules from $\left(E^{\prime}, \theta^{\prime}\right)$ to $(E, \theta)$. It is easy to check that it yields an isomorphism of $\tau(E, \theta, F i l, \phi, \iota)$ in the latter category. The functorial property of $\tau$ is easily verified.

This completes the Higgs correspondence in positive characteristic. In the following we deduce from it some direct consequences.

\section{Crystalline $\mathbb{F}_{p^{f}}$-representations.}

Let $X / W$ be a smooth proper scheme over $W$. An $\mathbb{F}_{p^{f}}$-representation of $\pi_{1}\left(X_{K}\right)$ is said to be crystalline if it is crystalline as an $\mathbb{F}_{p}$-representation by restriction of scalar. In other words, a crystalline $\mathbb{F}_{p^{f}}$-representation is a crystalline $\mathbb{F}_{p^{-}}$

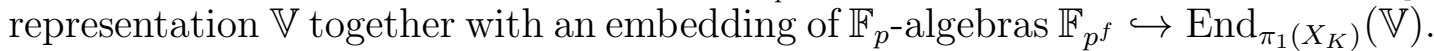
Similarly, one has the notion of crystalline $W_{n}\left(\mathbb{F}_{p^{f}}\right)$-representation for $n \in \mathbb{N} \cup$ $\{\infty\}$. The following corollary is immediate from Theorem 2.3 and Theorem 3.2 .

Corollary 3.10. Let $X / W$ be a smooth proper scheme. Assume $w \leq p-2$. There is an equivalence of categories between the category of crystalline $\mathbb{F}_{p^{f} \text { - }}$ representations of $\pi_{1}\left(X_{K}\right)$ with Hodge-Tate weight $\leq w$ and the category of $f$ periodic Higgs-de Rham flows of level $\leq w$ over $X_{1}$.

For an object $\left(E, \theta, F i l_{0}, \cdots, F i l_{f-1}, \phi\right) \in H D F_{w, f}\left(X_{2} / W_{2}\right)$, we define its shift and lengthening as follows: note for $\left(E_{f}, \theta_{f}\right)=G r_{F i l_{f-1}}\left(H_{f-1}, \nabla_{f-1}\right), C_{1}^{-1}(\phi)$ induces the pullback filtration $\left(C_{1}^{-1}(\phi)\right)^{*} F i l_{0}$ on $C_{1}^{-1}\left(E_{f}, \theta_{f}\right)$ and an isomorphism of graded Higgs modules $\operatorname{GrC}_{1}^{-1}(\phi)$ on the gradings. Then it is easy to check that the tuple

$$
\left(E_{1}, \theta_{1}, F i l_{1}, \cdots, F i l_{f-1}, C_{1}^{-1}(\phi)^{*} F i l_{0}, G r C_{1}^{-1}(\phi)\right)
$$

is an object in $H D F_{w, f}\left(X_{2} / W_{2}\right)$, which we call the shift of $\left(E, \theta, F i l_{0}, \cdots, F i l_{f-1}, \phi\right)$. For any multiple $l f, l \geq 1$, we can lengthen $\left(E, \theta, F i l_{0}, \cdots, F i l_{f-1}, \phi\right)$ to an object of $H D F_{w, l f}\left(X_{2} / W_{2}\right)$ : similar to above, we can inductively define the induced filtration on $\left(H_{j}, \nabla_{j}\right), f \leq j \leq l f-1$ from $F i l_{i} \mathrm{~s}$ via $\phi$. One has the induced isomorphism of graded Higgs modules

$$
\left(G r C_{1}^{-1}\right)^{l^{\prime} f}(\phi):\left(E_{\left(l^{\prime}+1\right) f}, \theta_{\left(l^{\prime}+1\right) f}\right) \cong\left(E_{l^{\prime} f}, \theta_{l^{\prime} f}\right), \quad 0 \leq l^{\prime} \leq l-1 .
$$

The isomorphism $\phi_{l}:\left(E_{l f}, \theta_{l f}\right) \cong\left(E_{0}, \theta_{0}\right)$ is defined to be the composite of them. The obtained object $\left(E, \theta, F i l_{0}, \cdots, F i l_{l f-1}, \phi_{l}\right)$ is called the $l-1$-th lengthening of $\left(E, \theta, F i l_{0}, \cdots, F i l_{f-1}, \phi\right)$. The following result will be obvious from the proof of Theorem 3.2

Corollary 3.11. Notation as in Corollary 3.10. Let $\rho$ be the corresponding crystalline $\mathbb{F}_{p^{f}}$-representation to $\left(E, \theta, F i l_{0}, \cdots, F i l_{f-1}, \phi\right)$. Then the followings are true: 
(i) The shift of $\left(E, \theta, F i l_{0}, \cdots, F i l_{f-1}, \phi\right)$ corresponds to $\rho^{\sigma}=\rho \otimes_{\mathbb{F}_{p f}, \sigma} \mathbb{F}_{p^{f}}$, the $\sigma$-conjugation of $\rho$. Here $\sigma \in \operatorname{Gal}\left(\mathbb{F}_{p^{f}} \mid \mathbb{F}_{p}\right)$ is the Frobenius element.

(ii) For $l \in \mathbb{N}$, the $l-1$-th lengthening of $\left(E, \theta, F i l_{0}, \cdots, F i l_{f-1}, \phi\right)$ corresponds to the extension of scalar $\rho \otimes_{\mathbb{F}_{p f}} \mathbb{F}_{p^{l f}}$.

Locally freeness of preperiodic Higgs modules.

In the following, we explain that a preperiodic Higgs module is locally free, a posteriori property.

Proposition 3.12. Periodic Higgs module is locally free.

Proof. Let $(E, \theta)$ be a periodic Higgs module. Then a periodic Higgs-de Rham flow with its leading term $(E, \theta)$ gives an object in the category $H D F_{w, f}\left(X_{2} / W_{2}\right)$ for some $f$. Let $(H, \nabla, F i l, \Phi, \iota)$ be the corresponding object in $M F_{[0, w], f}^{\nabla}\left(X_{2} / W_{2}\right)$ after Theorem [3.2. The proof of [4, Theorem 2.1] (cf. page 32 loc. cit.) asserts that $F i l$ is a filtration of locally free subsheaves of $H$ and locally split, which implies that $G r_{F i l} H$ is locally free. It follows immediately that $(E, \theta)$ is also locally free.

A. Langer [20, Proposition $1.1 \S 5.3$ ] has obtained the following enhancement of the previous result (notice however that the assumption that rank $E \leq p$ in the cited statement on the Higgs module is in our case unnecessary due to a slightly different definition of Higgs-de Rham flow).

Corollary 3.13 (Langer). Preperiodic Higgs module is locally free.

Proof. It follows from Corollary 3.12 and Lemma 3 [20].

Corollary 3.14. The Griffiths transverse filtrations in a preperiodic Higgs-de Rham flow are filtrations by locally free subsheaves and locally split.

Proof. The periodic case has been explained in the proof of Proposition 3.12. But it also follows from Corollary 3.13 , by induction on the level of filtrations. So does the preperiodic case.

\section{Inverse CARTier transform OVER A TRUnCATED WitT Ring}

The inverse Cartier transform of Ogus-Vologodsky [25] has played a pivotal role in the notion of a (periodic) Higgs-de Rham flow in characteristic $p$. In order to obtain the analogous notion of (periodic) Higgs-de Rham flow over a truncated Witt ring, we need to construct a lifting of the inverse Cartier transform. In this section, $X_{n}$ is a smooth scheme over $W_{n}$ and $X_{n+1}$ is a $W_{n+1}$-lifting of $X_{n}$.

An anonymous referee has kindly pointed to us that the work of A. Shiho [29] is related to our construction below. Recall, for each $n \in \mathbb{N}, S_{n}=\operatorname{Spec} W_{n}$ and $F_{S_{n}}: S_{n} \rightarrow S_{n}$ the Frobenius automorphism. In [29] Shiho constructs a functor from the category of quasi-nilpotent Higgs modules on $X_{n}^{(n)}$ to the category of quasi-nilpotent flat modules on $X_{n}$, where $X_{n}$ is a smooth scheme over $W_{n}$ and $X_{n}^{(n)}=X_{n} \times_{F_{S_{n}}^{n}} S_{n}$, under the assumption that $X_{n}^{(m)}=X_{n} \times_{F_{S_{n}}^{m}} S_{n}, 0 \leq m \leq n$ 
admits a smooth lifting $X_{n+1}^{(m)}$ to $S_{n+1}$ and the Frobenius liftings $F_{n+1}^{m}: X_{n+1}^{(m-1)} \rightarrow$ $X_{n+1}^{(m)}$ over $S_{n+1}$ exist. The functor is a nice $p$-adic reincarnation of the notion of $\lambda$-connection in complex differential geometry. However, the assumption on the Frobenius lifting is very restrictive for a projective $W_{n}$-scheme, which is however the basic assumption to formulate the semistability for Higgs modules. For example, a smooth projective curve over $W_{2}$ admits no Frobenius lifting once its genus is greater than one. Our construction was inspired by the fact that Ogus-Vologodsky's construction extends the theory of strict $p$-torsion Fontaine modules (see $\S 4$ [25], see also [26], 13]). We have worked out a generalization for sub strict $p^{n}$-torsion Fontaine modules in [28] and the current construction is then a further generalization (without assuming the existence of an ambient strict $p^{n}$-torsion Fontaine module).

Let us put $X_{n}^{\prime}=X_{n} \times_{F_{S_{n}}} S_{n}$. Then $X_{n+1}^{\prime}$ is a smooth lifting of $X_{n}^{\prime}$ over $W_{n+1}$. The $\bmod p^{n-1}$ reduction $X_{n} \otimes \mathbb{Z} / p^{n-1} \mathbb{Z}$ of $X_{n}$ is denoted by $X_{n-1}$. Similarly for $X_{n-1}^{\prime}$. Let us introduce a category $\mathcal{H}\left(X_{n}^{\prime}\right)$ of Higgs modules over $X_{n}^{\prime}$ as follows: an object is given by a tuple

$$
(E, \theta, \bar{H}, \bar{\nabla}, \overline{F i l}, \bar{\psi}),
$$

where $(E, \theta)$ is a graded Higgs module over $X_{n}^{\prime}$ of exponent $\leq p-2,(\bar{H}, \bar{\nabla}, \overline{F i l})$ a de Rham module over $X_{n-1}^{\prime}$ with the level of Hodge filtration $\leq p-2$ and

$$
\bar{\psi}: G r_{\overline{F i l}}(\bar{H}, \bar{\nabla}) \cong(\bar{E}, \bar{\theta}):=(E, \theta) \otimes \mathbb{Z} / p^{n-1} \mathbb{Z}
$$

an isomorphism of graded Higgs modules over $X_{n-1}^{\prime}$. The morphism in the category is defined in the obvious way. For $n=1$, the above tuple is reduced to a nilpotent graded Higgs module over $X_{1}^{\prime}$ of exponent $\leq p-2$. So $\mathcal{H}\left(X_{1}^{\prime}\right)$ is a full subcategory of $H I G_{p-1}\left(X_{1}^{\prime}\right)$.

Theorem 4.1. Notation as above. There exists a functor $\mathcal{C}_{n}^{-1}$ from the category $\mathcal{H}\left(X_{n}^{\prime}\right)$ to the category $\operatorname{MIC}\left(X_{n}\right)$ of flat modules over $X_{n}$ such that $\mathcal{C}_{n}^{-1}$ lifts $\mathcal{C}_{n-1}^{-1}$ and such that $\mathcal{C}_{1}^{-1}$ agrees with the inverse Cartier transform $C_{\mathcal{X} / \mathcal{S}}^{-1}$ of OgusVologodsky [25] with $(\mathcal{X}, \mathcal{S})=\left(X_{1} / k, X_{2}^{\prime} / W_{2}\right)$.

We shall also introduce an intermediate category $\widetilde{M I C}\left(X_{n}^{\prime}\right)$ which we call the category of twisted flat modules over $X_{n}^{\prime}$. The construction of the functor $\mathcal{C}_{n}^{-1}$ consists of constructing the first functor $\mathcal{T}_{n}: \mathcal{H}\left(X_{n}^{\prime}\right) \rightarrow \widetilde{M I C}\left(X_{n}^{\prime}\right)$ and the second functor $\mathcal{F}_{n}: \widetilde{M I C}\left(X_{n}^{\prime}\right) \rightarrow M I C\left(X_{n}\right)$. The category $\widetilde{M I C}\left(X_{n}^{\prime}\right)$ is closely related to the category of quasi-nilpotent $\mathcal{O}_{X_{n}^{\prime}}$-modules with integrable $p$-connections of Shiho [29] which we shall explain later. The motivation to introduce this new category is mainly because of the necessity to make sense of $p$-powers in the denominators appearing in the Taylor formula (4.16.1).

Let $X$ be a smooth scheme over $S_{n}$. First recall that a Lie algebroid on $X$ is a locally free $\mathcal{O}_{X}$-module $\mathcal{A}$ equipped with a skew-symmetric $\mathcal{O}_{X}$-bilinear pairing

$$
[\cdot, \cdot]_{\mathcal{A}}: \mathcal{A} \times \mathcal{A} \rightarrow \mathcal{A}
$$


satisfying the Jacobi identity and an action of $\mathcal{A}$ on $\mathcal{O}_{X}$ by derivations (so-called anchor map) which is given by an $\mathcal{O}_{X^{-}}$linear morphism of Lie algebras

$$
\alpha: \mathcal{A} \rightarrow T_{X}
$$

subject to the compatibility condition (Leibniz rule)

$$
[x, f y]_{\mathcal{A}}=\alpha(x)(f) y+f[x, y]_{\mathcal{A}} .
$$

Let us consider the following Lie algebroid $\left(T_{X}, \alpha,\{\cdot, \cdot\}:=p[\cdot, \cdot]\right)$, with the anchor $\alpha=p \cdot I d: T_{X} \rightarrow T_{X}$ and $\left\{D_{1}, D_{2}\right\}=p\left[D_{1}, D_{2}\right]$ for any local sections $D_{i}, i=1,2$ of $T_{X}$, where $[\cdot, \cdot]$ is the usual Lie bracket for the tangent sheaf. Let $\mathcal{D}_{X}^{(-1)}$ denote the sheaf of enveloping algebras of the Lie algebroid $\left(T_{X}, \alpha,\{\cdot, \cdot\}\right)$. Thus $\mathcal{D}_{X}^{(-1)}$ is generated by the algebra of functions $\mathcal{O}_{X}$ and the $\mathcal{O}_{X}$-module of derivations $T_{X}$, subject to the module and commutator relations

$$
f \cdot D=f D, \quad D \cdot f-f \cdot D=p D(f), \quad D \in T_{X}, \quad f \in \mathcal{O}_{X},
$$

and the Lie algebroid relation

$$
D_{1} \cdot D_{2}-D_{2} \cdot D_{1}=\left\{D_{1}, D_{2}\right\}, \quad D_{1}, D_{2} \in T_{X} .
$$

Next we introduce a sheaf of twisted differential operators on $X$ as follows.

Definition 4.2. Notation as above. Let $U \subset X$ be an open affine subset over $S_{n}$. Set $\widetilde{\mathcal{D}}_{X}(U)$ to be the algebra generated over $\mathcal{D}_{X}^{(-1)}(U)$ by symbols

$$
\left\{\gamma_{m}\left(D_{1}, \cdots, D_{p-1+m}\right) \mid \text { for } m \in \mathbb{N} \text {, and any } D_{1}, \cdots D_{p-1+m} \in T_{X}(U)\right\} \text {, }
$$

subject to the following six relations:

(1) $p^{m} \cdot \gamma_{m}\left(D_{1}, \cdots, D_{p-1+m}\right)=D_{1} \cdots D_{p-1+m}$;

(2) $\gamma_{m}\left(D_{1}, \cdots, \alpha D_{i}+\alpha^{\prime} D_{i}^{\prime}, \cdots D_{p-1+m}\right)=\alpha \cdot \gamma_{m}\left(D_{1}, \cdots, D_{i}, \cdots, D_{p-1+m}\right)$ $+\alpha^{\prime} \cdot \gamma_{m}\left(D_{1}, \cdots, D_{i}^{\prime}, \cdots, D_{p-1+m}\right), \alpha, \alpha^{\prime} \in W_{n}, D_{i}, D_{i}^{\prime} \in T_{X}(U)$;

(3) for $m \geq 1, \gamma_{m}\left(D_{1}, \cdots, D_{p-1+m}\right) \cdot f=f \cdot \gamma_{m}\left(D_{1}, \cdots, D_{p-1+m}\right)$

$+\sum_{i=1}^{p-2+m} \gamma_{m-1}\left(D_{1}, \cdots, D_{i-1}, D_{i}(f) D_{i+1}, \cdots, D_{p-1+m}\right)+$ $\gamma_{m-1}\left(D_{1}, \cdots, D_{p-2+m}\right) \cdot D_{p-1+m}(f)$;

(4) $\gamma_{m}\left(D_{1}, \cdots, D_{i}, D_{i+1}, \cdots, D_{p-1+m}\right)=\gamma_{m}\left(D_{1}, \cdots, D_{i+1}, D_{i}, \cdots, D_{p-1+m}\right)$

$+\gamma_{m-1}\left(D_{1}, \cdots, D_{i-1},\left[D_{i}, D_{i+1}\right], D_{i+2}, \cdots, D_{p-1+m}\right)$;

(5) $\gamma_{m_{1}}\left(D_{1}, \cdots, D_{p-1+m_{1}}\right) \cdot \gamma_{m_{2}}\left(D_{p+m_{1}}, \cdots, D_{2 p-2+m_{1}+m_{2}}\right)$

$=p^{p-1} \gamma_{p-1+m_{1}+m_{2}}\left(D_{1}, \cdots, D_{2 p-2+m_{1}+m_{2}}\right)$;

(6) $\gamma_{m}\left(D_{1}, \cdots, D_{p-1+m}\right) \cdot D_{p+m}=D_{1} \cdot \gamma_{m}\left(D_{2}, \cdots, D_{p+m}\right)$

$=p \cdot \gamma_{m+1}\left(D_{1}, \cdots, D_{p-1+m}, D_{p+m}\right)$.

The sheaf of twisted differential operators $\widetilde{\mathcal{D}}_{X}$ is the associated sheaf to the presheaf $U \mapsto \widetilde{\mathcal{D}}_{X}(U)$.

The above definition is to legitimate the $p$-powers appearing in the denominators of differential operators. In particular, one may regard $\gamma_{m}\left(D_{1}, \cdots, D_{p-1+m}\right)$ as a symbol for $\frac{D_{1} \cdots D_{p-1+m}}{p^{m}}$. Since $\widetilde{\mathcal{D}}_{X}$ contains $\mathcal{O}_{X}$ as a subsheaf of algebras, it has a natural left $\mathcal{O}_{X}$-module structure. Thus it contains $T_{X}$ as a left $\mathcal{O}_{X}$-submodule. 
Definition 4.3. A twisted connection on an $\mathcal{O}_{X}$-module $H$ is a $W_{n}$-morphism between sheaves of $W_{n}$-algebras

$$
\tilde{\nabla}: \widetilde{\mathcal{D}}_{X} \rightarrow \operatorname{End}_{W_{n}}(H)
$$

extending the structural morphism $\mathcal{O}_{X} \rightarrow \operatorname{End}_{W_{n}}(H)$.

A coherent $\mathcal{O}_{X}$-module equipped with a twisted connection is said to a twisted flat module. Let $\widehat{M I C}(X)$ be the category of twisted flat modules over $X$. An explanation of the relation with the notion of an $\mathcal{O}_{X}$-module with integrable $p$-connection is in order. We cite the following definition from Shiho [29]:

Definition 4.4 ([29, Definition 1.1-1.2,1.5]). Notation as above. A p-connection $\nabla$ on an $\mathcal{O}_{X}$-module $H$ is an $W_{n}$-linear map $\nabla: H \rightarrow H \otimes \Omega_{X}$, such that

$$
\nabla(f h)=p \cdot d f \otimes h+f \nabla(h), \quad f \in \mathcal{O}_{X}, h \in H .
$$

It is said to be integrable if $\nabla_{1} \circ \nabla=0$, where

$$
\nabla_{1}: H \otimes \Omega_{X} \rightarrow H \otimes \Omega_{X}^{2}
$$

is the $W_{n}$-linear map defined by via the formula

$$
\nabla_{1}(h \otimes \omega)=\nabla(h) \wedge \omega+p h \otimes d \omega .
$$

Moreover, an integrable $p$-connection $(H, \nabla)$ is quasi-nilpotent if locally $\theta^{a}=0$ once $|a| \geq N$ for some natural number $N$ where $\theta$ is the connection one-form $\nabla(e)=\sum_{i} \theta_{i}(e) d t_{i}$ with respect to a set of étale local coordinates $\left\{t_{1}, \cdots, t_{d}\right\}$ of $X$ and $\theta^{a}=\prod_{i} \theta_{i}^{a_{i}}$ for a multi-index $a=\left(a_{1}, \cdots, a_{d}\right) \in \mathbb{N}^{d}$ and $|a|=\sum_{i} a_{i}$.

In the above definition, the natural number $N$ depends on the local expression of a local section $e$. However, in Lemma 1.6 [29], an integrable $p$-connection $\nabla$ on $H$ being quasi-nilpotent is shown to be independent of choices of local coordinates. Let us denote the category of $\mathcal{O}_{X}$-modules with $p$-connections by $M C^{(-1)}(X)$ and the integrable ones by $M I C^{(-1)}(X)$. To our purpose, we also need to introduce a level structure on the quasi-nilpotency of an integrable $p$-connection.

Definition 4.5. Notation as above. Let $(H, \nabla)$ be a quasi-nilpotent $\mathcal{O}_{X}$-module with an integrable $p$-connection. It is said to be quasi-nilpotent of level $\leq m$ if

$$
\nabla_{D_{1}} \circ \cdots \circ \nabla_{D_{m+1}}=0, \quad \text { for any } D_{1}, \cdots D_{m+1} \in T_{X} .
$$

Denote the category of quasi-nilpotent $\mathcal{O}_{X}$-modules with an integrable $p$-connection of level $\leq m$ by $M I C_{m}^{(-1)}(X)$.

We shall point out that it is rather subtle to read the level structure in terms of local coordinates. Now there is a functor from $\widehat{M I C}(X)$ to $M I C_{p-2+n}^{(-1)}(X)$ : given a twisted connection $\tilde{\nabla}$ on $H$, one considers only the restriction to $T_{X} \subset \tilde{\mathcal{D}}_{X}$ which yields a $W_{n}$-linear morphism

$$
\nabla:=\left.\tilde{\nabla}\right|_{T_{X}}: H \rightarrow H \otimes \Omega_{X} .
$$


It follows directly from the definition that this is indeed an integrable $p$-connection and quasi-nilpotent of level $\leq p-2+n$. Notice that the integrability amounts to the relation

$$
\nabla\left(D_{1}\right) \circ \nabla\left(D_{2}\right)-\nabla\left(D_{2}\right) \circ \nabla\left(D_{1}\right)=\nabla\left(\left\{D_{1}, D_{2}\right\}\right), \quad D_{1}, D_{2} \in T_{X}
$$

For $m \geq p$, we may write

$$
\frac{\nabla\left(D_{1}\right) \circ \cdots \circ \nabla\left(D_{m}\right)}{m !}=\frac{p^{m+1-p}}{m !} \cdot \widetilde{\nabla}\left(\gamma_{m+1-p}\left(D_{1}, \cdots, D_{m}\right)\right),
$$

and as the factor $\frac{p^{m+1-p}}{m !}$ converges to zero $p$-adically as $m$ goes to the infinity, $\frac{\nabla\left(D_{1}\right) \circ \cdots \circ \nabla\left(D_{m}\right)}{m !} \rightarrow 0$ when $m \rightarrow \infty$. This convergence property is crucial in the construction of our second functor $\mathcal{F}_{n}$. As a side remark, we do not know whether the above functor from $\widehat{M I C}(X)$ to $M I C_{p-2+n}^{(-1)}(X)$ is essentially surjective (but it is not faithful). Later, we shall come back to this point again.

Now we can proceed to the construction of our first functor $\mathcal{T}_{n}: \mathcal{H}\left(X_{n}^{\prime}\right) \rightarrow$ $\widehat{M I C}\left(X_{n}^{\prime}\right)$. We shall present two approaches in the following. The first approach, which is our original approach, is a method of "local lifting-global gluing" which may be more familiar to a reader whose background is in complex algebraic geometry. The second approach is based on a beautiful construction suggested by the referee who kindly allows us to reproduce his/her idea here. This direct approach is much more transparent.

First approach.

Let $X$ be a smooth scheme over $S_{n}$. Let $\bar{X}:=X \otimes \mathbb{Z} / p^{n-1} \mathbb{Z}$ be its reduction $\bmod p^{n-1}$. Since our arguments rely heavily on local calculations using a basis of local sections of an $\mathcal{O}_{X}$-module, we will restrict ourselves in this approach to the full subcategory $\mathcal{H}_{l f}(X) \subset \mathcal{H}(X)$ consisting of locally free objects. However, this restriction is caused mainly for simplicity in the local arguments. For a general coherent object, we may use a set of minimal generators with possible relations for a coherent object locally. Also, we need to introduce some other categories which will be used only in this approach. The category $\mathcal{H}^{n i}(X)\left(\operatorname{resp} . \mathcal{H}_{l f}^{n i}(X)\right)$ is a variant of $\mathcal{H}(X)$ (resp. $\left.\mathcal{H}_{l f}(X)\right)$ : its object is also a tuple $(E, \theta, \bar{H}, \bar{\nabla}, \overline{F i l}, \bar{\psi})$; but the integrabilities on $\theta$ and $\bar{\nabla}$ are not required. For brevity, an object in $\mathcal{H}(X)$ or $\mathcal{H}^{n i}(X)$ is written as $(E, \bar{H})$. Second, let $M C F_{p-2}(X)$ be a category of filtered $\mathcal{O}_{X}$-modules equipped with (not necessarily integrable) connections: its object is a triple $(H, \nabla, F i l)$, where $H$ is a locally free $\mathcal{O}_{X}$-module, $\nabla$ is a $W_{n^{-}}$ linear connection on $H$, and $F i l$ a finite exhaustive decreasing filtration of locally free $\mathcal{O}_{X}$-submodules on $H$ of level $\leq p-2$ which is locally split and satisfies Griffiths' transversality. There is a diagram of these categories connected by 
natural functors:

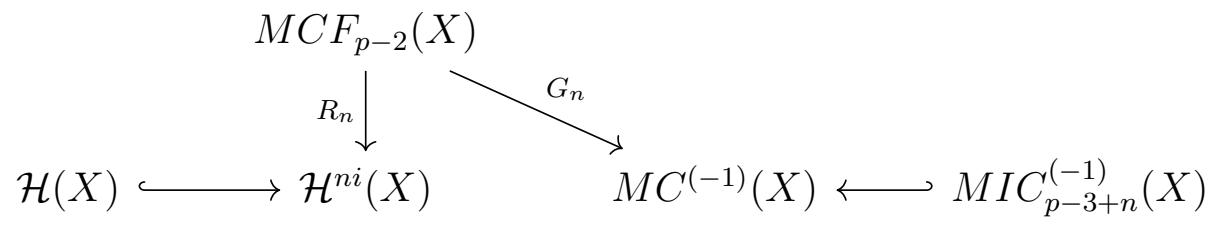

The functor $R_{n}$ is the obvious one: to $(H, \nabla, F i l)$, one associates the graded Higgs module $(E, \theta)=G r_{F i l}(H, \nabla)$, which is locally free by the assumption on the filtration, and also $(\bar{H}, \bar{\nabla}, \overline{F i l})$, its $\bmod p^{n-1}$ reduction. One notices that there is a natural isomorphism of graded Higgs modules

$$
\bar{\psi}: G r_{\overline{F i l}}(\bar{H}, \bar{\nabla}) \cong G r_{F i l}(H, \nabla) \otimes \mathbb{Z} / p^{n-1} \mathbb{Z} .
$$

The functor $G_{n}$ is a variant of a construction due to Faltings [4, Ch.II], which originates from [7] in the case $X=S_{n}$. Given an object $(H, \nabla, F i l) \in M C F_{p-2}(X)$, the object $(\tilde{H}, \tilde{\nabla})=G_{n}(H, \nabla, F i l)$ is defined as follows: $\tilde{H}$ is the cokernel of the first map of the following exact sequence

$$
\oplus_{i} F i l^{i} \stackrel{[-1]-p \cdot I d}{\longrightarrow} \oplus_{i} F i l^{i} \stackrel{\rho}{\longrightarrow} \tilde{H} \rightarrow 0,
$$

where $[-1]:=\oplus_{i}\left(F i l^{i} \hookrightarrow F i l^{i-1}\right), I d$ denotes the identity map, and $\rho$ is the natural projection map. Here we have extended the filtration so that

$$
F i l^{i}=F i l^{0}, i \leq-1, \quad F i l^{j}=0, j \geq p .
$$

Consider the $W_{n}$-linear map

$$
\nabla^{\prime}:=\left.\oplus_{i} \nabla\right|_{F i l^{i}},\left.\quad \nabla\right|_{F i l^{i}}: F i l^{i} \rightarrow F i l^{i-1} \otimes \Omega_{X} .
$$

The image $\left.([-1]-p \cdot I d)\left(\oplus_{i} F i l^{i}\right) \subset \oplus_{i} \nabla\right|_{F i l^{i}}$ being preserved, $\nabla^{\prime}$ induces a $W_{n^{-}}$ linear map

$$
\tilde{\nabla}: \tilde{H} \rightarrow \tilde{H} \otimes \Omega_{X}
$$

One checks immediately that $\tilde{\nabla}$ is indeed a $p$-connection.

First, we show that the functor $R_{n}$ is locally essentially surjective.

Lemma 4.6. Let $X$ be a smooth affine scheme over $S_{n}$. Let $(E, \bar{H})$ be an object in $\mathcal{H}_{l f}^{n i}(X)$. Suppose each component of $E=\oplus_{k} E_{k}$ is a free $\mathcal{O}_{X}$-module. Then there exists an object $(H, \nabla, F i l) \in M C F_{p-2}(X)$ such that $R_{n}(H, \nabla, F i l)=(E, \bar{H})$.

Proof. Write $E=\oplus_{k=0}^{w} E^{w-k, k}, \theta=\oplus_{k=0}^{w-1} \theta_{w}$, and for $0 \leq k \leq w$ take a set of basis $\left\{e_{k}\right\}$ for the $\mathcal{O}_{X}$-module $E^{w-k, k}$. Under the basis, $\theta_{k}$ is expressed in terms of a matrix of differential one-forms which we write again by $\theta_{k}$. Put the bar over $\theta_{k}$ as well as $\left\{e_{k}\right\}$ to mean their mod $p^{n-1}$-reduction. Then take the basis $\left\{f_{k}^{\prime}\right\}_{0 \leq k \leq w}$ of $G r_{\overline{F i l}}(\bar{H})$ such that

$$
\bar{\psi}\left(f_{k}^{\prime}\right)=\bar{e}_{k}
$$


Also choose a basis $\left\{\bar{f}_{k}\right\}_{0 \leq k \leq w}$ of $\bar{H}$ such that its image in $\operatorname{Gr} \overline{F i l}(\bar{H})$ is $\left\{f_{k}^{\prime}\right\}_{0 \leq k \leq w}$. By Griffiths' transversality, the connection matrix $\left(\bar{a}_{i j}\right)$ representing the connection $\bar{\nabla}$ under the basis $\left\{\bar{f}_{k}\right\}$, i.e.,

$$
\bar{\nabla}\left(\bar{f}_{i}\right)=\sum_{j} \bar{a}_{i j} \bar{f}_{j}
$$

has the property $\bar{a}_{i j}=0, j>i+1$. Now we take a matrix of differential oneforms $\left(a_{i j}\right)$ over $X$ as follows: for $i \geq j$, take any lift $a_{i j}$ of $\bar{a}_{i j}$; for $j=i+1$, take $a_{i j}=\theta_{i}$; for $j>i+1$, take $a_{i j}=0$. Now let $H$ be the free $\mathcal{O}_{X}$-module generated by $\left\{f_{k}\right\}_{0 \leq k \leq w}$ whose $\bmod p^{n-1}$ reduction are $\left\{\bar{f}_{k}\right\}_{0 \leq k \leq w}$ and let $F i l^{w-k}$ be the submodule freely generated by the elements $\left\{f_{j}\right\}_{0 \leq j \leq k}$, which gives the filtration Fil on $H$. Then a connection $\nabla$ on $H$ is determined by the formula $\nabla\left(f_{i}\right)=\sum_{j} a_{i j} f_{j}$. The so-constructed triple $(H, \nabla, F i l)$ is indeed an object in $M C F_{p-2}(X)$ lifting $(E, \bar{H})$, i.e., $R_{n}(H, \nabla, F i l)=(E, \bar{H})$ as required.

Next we show the following

Lemma 4.7. If $R_{n}(H, \nabla, F i l)$ lies in the full subcategory $\mathcal{H}_{l f}(X) \subset \mathcal{H}_{l f}^{n i}(X)$, then $G_{n}(H, \nabla, F i l)$ lies in the full subcategory $M I C_{p-3+n}^{(-1)}(X)$.

Proof. Set $(\tilde{H}, \tilde{\nabla}):=G_{n}(H, \nabla, F i l)$. Once the integrability of $\tilde{\nabla}$ is verified, the statement on quasi-nilpotency of level $\leq p-3+n$ follows directly from the definition. As the integrability is a local property, we assume a set of étale local coordinate $\left\{t_{1}, \cdots, t_{d}\right\}$ of $X$ and a filtered basis $\left\{f_{k}\right\}_{0 \leq k \leq w}$ of $H$. It suffices to check that for $1 \leq i, j \leq d, \tilde{\nabla}\left(\partial t_{i}\right)$ commutates with $\tilde{\nabla}\left(\partial t_{j}\right)$.

Let $\tilde{f}_{k}$ be the image of $f_{k}$ (elements of $\left.F i l^{k}\right)$ in $\tilde{H}$. Then $\left\{\tilde{f}_{k}\right\}_{0 \leq k \leq w}$ forms a basis of $\tilde{H}$. As in Lemma (4.6), we may assume the matrix $A:=\left(a_{i j}\right)$ of $\nabla$ under the basis $\left\{f_{k}\right\}_{0 \leq k \leq w}$ has the property that $a_{i j}=0$ for $j>i+1$. Then the matrix of $\tilde{\nabla}$ under the basis $\left\{\tilde{f}_{k}\right\}_{0 \leq k \leq m}$ is $\tilde{A}=\left(\tilde{a}_{i j}\right)$, with $\tilde{a}_{i j}=p^{i+1-j} a_{i j}$ for $j \leq i+1$, and the rest are zero. Then $\tilde{\nabla}\left(\partial t_{i}\right) \circ \tilde{\nabla}\left(\partial t_{j}\right)$ under the basis $\left\{\tilde{f}_{k}\right\}$ is represented by the matrix

$$
p \partial t_{i}\left(\partial t_{j} \tilde{A}\right)+\left(\partial t_{j} \tilde{A}\right) \cdot\left(\partial t_{i} \tilde{A}\right) .
$$

It remains to show the equality for all $i, j$ :

$$
p \partial t_{i}\left(\partial t_{j} \tilde{A}\right)+\left(\partial t_{j} \tilde{A}\right) \cdot\left(\partial t_{i} \tilde{A}\right)-p \partial t_{j}\left(\partial t_{i} \tilde{A}\right)-\left(\partial t_{i} \tilde{A}\right) \cdot\left(\partial t_{j} \tilde{A}\right)=0,
$$

For $0 \leq r \leq w-2$ with $t=r+1$ and $s=r+2$, the corresponding entry in the above equality means

$$
\left(\partial t_{i} a_{r t}\right)\left(\partial t_{j} a_{t s}\right)-\left(\partial t_{j} a_{r t}\right)\left(\partial t_{i} a_{t s}\right)=0
$$

which is actually equivalent to the integrability of the Higgs field $\theta$; For $0 \leq r, s \leq$ $w$ with $s \leq r+1$, it means then

$$
p^{r+2-s}\left(\partial t_{i} \wedge \partial t_{j}\right)\left(d a_{r s}\right)=p^{r+2-s} \sum_{t=0}^{w}\left[\left(\partial t_{i} a_{r t}\right)\left(\partial t_{j} a_{t s}\right)-\left(\partial t_{j} a_{r t}\right)\left(\partial t_{i} a_{t s}\right)\right] .
$$


As $r+2-s \geq 1$, the above equation is implied by the following equation, which is equivalent to the integrability of $\bar{\nabla}$ :

$$
\left(\partial \bar{t}_{i} \wedge \partial \bar{t}_{j}\right)\left(d \bar{a}_{r s}\right)=\sum_{t=0}^{w}\left[\left(\partial \bar{t}_{i} \bar{a}_{r t}\right)\left(\partial \bar{t}_{j} \bar{a}_{t s}\right)-\left(\partial \bar{t}_{j} \bar{a}_{r t}\right)\left(\partial \bar{t}_{i} \bar{a}_{t s}\right)\right]
$$

Here $\left\{\bar{t}_{1}, \cdots, \bar{t}_{d}\right\}$ means the induced set of étale local coordinates on $\bar{X}$.

We shall take a step further to land the object $G_{n}(H, \nabla, F i l)$ in the last lemma in the category of twisted connections.

Lemma 4.8. Notation as above. Then the integrable $p$-connection of $G_{n}(H, \nabla, F i l)$ can be extended to a twisted connection in a functorial way.

Proof. If $(H, \nabla, F i l)$ is the mod $p^{n}$ reduction of an object over $W$, then it is clear how to extend $\tilde{\nabla}$ to a twisted connection: for $\gamma_{m}\left(D_{1}, \cdots, D_{p-1+m}\right)$ with $D_{i} \in T_{X}$, one defines $\tilde{\nabla}\left(\gamma_{m}\left(D_{1}, \cdots, D_{p-1+m}\right)\right)$ to be $\frac{\tilde{\nabla}_{D_{1} \cdots \cdots \circ} \tilde{\nabla}_{D_{p-1+m}}}{p^{m}}$, which is defined by first lifting to $W$, then dividing $p^{m}$ and finally taking the reduction modulo $p^{n}$. The reason that $\tilde{\nabla}_{D_{1}} \circ \cdots \circ \tilde{\nabla}_{D_{p-1+m}}$ is divisible by $p^{m}$ is examined as follows: by Griffiths' transversality, it follows that

$$
\nabla_{D_{m+1}}^{\prime} \circ \cdots \circ \nabla_{D_{p-1+m}}^{\prime}: F i l^{i} \rightarrow F i l^{i+1-p} .
$$

As $F i l^{j}=0$ for $j \geq w+1$, and $w \leq p-2$, one sees that the image of $\nabla_{D_{m+1}}^{\prime} \circ \cdots \circ$ $\nabla_{D_{p-1+m}}^{\prime}$ lies in $F i l^{i}$ with $i \leq-1$. In the quotient $\tilde{H}$, for $i \leq-1$, the image of each element in $F i l^{i-1}$ is equal to $p$ times the same element in $F i l^{i}$. Thus, since $\nabla^{\prime}$ always shifts the indices of direct factors of $\oplus_{i} F i l^{i}$ by minus one, the restriction of $\tilde{\nabla}$ to the image $\bar{F} i l^{-1}$ (which is isomorphic to $H$ ) of $F i l^{-1}$ in $\tilde{H}$ is divisible by $p$. On the other hand, this also gives us a way to extend $\tilde{\nabla}$ to an twisted connection in the general case. Indeed, we simply define $\tilde{\nabla}\left(\gamma_{m}\left(D_{1}, \cdots, D_{p-1+m}\right)\right)$ to be the composite $\frac{\nabla_{D_{1}}^{\prime}}{p} \circ \cdots \circ \frac{\nabla_{D_{m}}^{\prime}}{p} \circ \nabla_{D_{m+1}}^{\prime} \circ \cdots \circ \nabla_{D_{p-1+m}}^{\prime}$, where the symbol $\frac{\nabla_{D_{i}}^{\prime}}{p}$ is defined to be the map $\nabla_{D_{i}}$ on $\bar{F} i l^{-1} \subset \tilde{H}$. One verifies directly that this defines a twisted connection which extends $\tilde{\nabla}$.

We believe the truth of the following

Question 4.9. Can $M I C_{p-3+n}^{(-1)}(X)$ be realized as a full subcategory of $\widetilde{M I C}(X)$ ? We shall also provide the gluing morphisms.

Lemma 4.10. Let $\left(H_{i}, \nabla_{i}, F i l_{i}\right) \in M C F_{p-2}(X), i=1,2,3$. Suppose there are isomorphisms for $1 \leq i<j \leq 3$,

$$
\bar{f}_{i j}:\left(\bar{H}_{i}, \bar{\nabla}_{i}, \overline{F i l}_{i}\right) \cong\left(\bar{H}_{j}, \bar{\nabla}_{j}, \overline{F i l}_{j}\right), \quad f_{i j}^{G}: G r_{F i l_{i}}\left(H_{i}, \nabla_{i}\right) \cong G r_{F i l_{j}}\left(H_{j}, \nabla_{j}\right),
$$

satisfying

$$
\operatorname{Gr}\left(\bar{f}_{i j}\right)=f_{i j}^{G} \bmod p^{n-1} .
$$

Then there are isomorphisms in the category of $\widetilde{M I C}(X)$ between the objects $\left(\tilde{H}_{i}, \tilde{\nabla}_{i}\right):=G_{n}\left(H_{i}, \nabla_{i}, F i l_{i}\right)$

$$
\tilde{f}_{i j}:\left(\tilde{H}_{i}, \tilde{\nabla}_{i}\right) \cong\left(\tilde{H}_{j}, \tilde{\nabla}_{j}\right) .
$$


Moreover, if there are cocycle conditions

$$
\bar{f}_{13}=\bar{f}_{23} \circ \bar{f}_{12}, \quad f_{13}^{G}=f_{23}^{G} \circ f_{12}^{G},
$$

then $\left\{\tilde{f}_{i j}\right\}$ s also satisfy the cocycle condition

$$
\tilde{f}_{13}=\tilde{f}_{23} \circ \tilde{f}_{12} \text {. }
$$

Proof. For an $s \in F i l_{i}^{k} \backslash F i l_{i}^{k+1}$, we denote by $\tilde{s}$ (resp. $\hat{s}, \bar{s}$ ) its image under the natural map $F i l_{i}^{k} \rightarrow \tilde{H}_{i}$ (resp. Fil $l_{i}^{k} \rightarrow F i l_{i}^{k} / F i l_{i}^{k+1}, H_{i} \rightarrow \bar{H}_{i}$ ). Consider sections of $F i l_{j}^{k}$ whose images under the map $H_{j} \rightarrow \bar{H}_{j}$ are equal to $\bar{f}_{i j}(\bar{s})$ and under the map $F i l_{j}^{k} \rightarrow F i l_{j}^{k} / F i l_{j}^{k+1}$ equal to $f_{i j}^{G}(\hat{s})$ at the same time. As the difference of any two such sections lies in $p^{n-1} F i l_{j}^{k+1}$, they give rise to a unique section $\tilde{f}_{i j}(\tilde{s}) \in \tilde{H}_{j}$. So we define $\tilde{f}_{i j}: \tilde{H}_{i} \rightarrow \tilde{H}_{j}$ by sending $\tilde{s}$ to $\tilde{f}_{i j}(\tilde{s})$. It is straightforward to verify the well-definedness of $\tilde{f}_{i j}$ as well as the compatibility with twisted connections. The cocycle condition for $\left\{\tilde{f}_{i j}\right\}$ follows directly from the definition.

Now we can give the construction of the first functor. One shall notice that it does not require $X$ to be $W_{n+1}$-liftable.

Proposition 4.11. Let $X$ be a smooth scheme over $S_{n}$. Then there is a functor $\mathcal{T}_{n}$ from the category $\mathcal{H}_{l f}(X)$ to the category $\widehat{M I C}(X)$.

Proof. Let $(E, \bar{H})$ be an object in $\mathcal{H}_{l f}(X)$. Take an open affine covering $\mathcal{U}=$ $\left\{U_{i}\right\}_{i \in I}$ of $X$ such that $E$ and $\bar{H}$ are free modules over each $U_{i}$. By Lemma 4.6, we can take an object $\left(H_{i}, \nabla_{i}, F i l_{i}\right) \in M C F_{p-2}\left(U_{i}\right)$ for $U_{i} \in \mathcal{U}$ such that $R_{n}\left(H_{i}, \nabla_{i}, F i l_{i}\right)=\left.(E, \bar{H})\right|_{U_{i}}$ (we call $\left(H_{i}, \nabla_{i}, F i l_{i}\right)$ a local lifting). Since over $U_{i} \cap U_{j}$, the restrictions of $\left(H_{i}, \nabla_{i}, F i l_{i}\right)$ and $\left(H_{j}, \nabla_{j}, F i l_{j}\right)$ are both local liftings of $\left.(E, \bar{H})\right|_{U_{i} \cap U_{j}}$, there are isomorphisms by Lemma 4.10

$$
\tilde{f}_{i j}:\left.\left.G_{n}\left(H_{i}, \nabla_{i}, F i l_{i}\right)\right|_{U_{i} \cap U_{j}} \cong G_{n}\left(H_{j}, \nabla_{j}, F i l_{j}\right)\right|_{U_{i} \cap U_{j}}
$$

satisfying the cocycle condition on $U_{i} \cap U_{j} \cap U_{k}$. Thus gluing the local objects $\left\{G_{n}\left(H_{i}, \nabla_{i}, F i l_{i}\right)\right\}_{U_{i} \in \mathcal{U}}$ in $\widetilde{M I C}\left(U_{i}\right)$ via the isomorphisms $\left\{\tilde{f}_{i j}\right\}$ yields an object

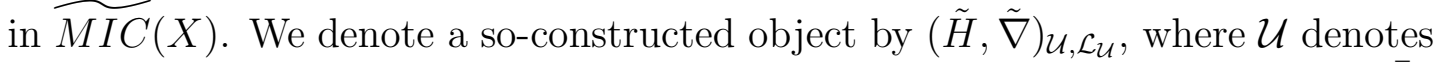
for an open affine covering of $X$ and $\mathcal{L}_{\mathcal{U}}$ consists of a local lifting of $(E, \bar{H})$ restricted to each $U \in \mathcal{U}$. We need to show that this object is independent of the choice of local liftings and the choice of open affine coverings up to canonical isomorphism. For two sets of local liftings $\mathcal{L}_{\mathcal{U}}^{1}=\left\{\left(H_{i}^{1}, \nabla_{i}^{1}, F i l_{i}^{1}\right)\right\}_{i \in I}$ and $\mathcal{L}_{\mathcal{U}}^{2}=$ $\left\{\left(H_{i}^{2}, \nabla_{i}^{2}, F i l_{i}^{2}\right)\right\}_{i \in I}$, Lemma 4.10 provides an isomorphism over each $U_{i} \in \mathcal{U}$ :

$$
\mu_{i}: G_{n}\left(H_{i}^{1}, \nabla_{i}^{1}, F i l_{i}^{* 1}\right) \cong G_{n}\left(H_{i}^{2}, \nabla_{i}^{2}, F i l_{i}^{* 2}\right)
$$

and over $U_{i} \cap U_{j}$, the equality $\tilde{f}_{i j}^{2} \circ \mu_{i}=\mu_{j} \circ \tilde{f}_{i j}^{1}$ holds. So the set of local isomorphisms $\left\{\mu_{i}\right\}_{i \in I}$ glues into a global isomorphism

$$
\mu:(\tilde{H}, \tilde{\nabla})_{\mathcal{U}, \mathcal{L}_{\mathcal{U}}} \cong(\tilde{H}, \tilde{\nabla})_{\mathcal{U}, \mathcal{L}_{\mathcal{U}}^{\prime}}
$$

One can further show that for any three choices of local liftings, the so-obtained isomorphisms satisfy the cocycle relation. So the object $(\tilde{H}, \tilde{\nabla})_{\mathcal{U}, \mathcal{L}_{\mathcal{U}}}$ is independent 
of the choice of local liftings up to canonical isomorphism and therefore can be denoted by $(\tilde{H}, \tilde{\nabla}) \mathcal{U}$. Next for any two choices $\mathcal{U}, \mathcal{U}^{\prime}$ of open affine coverings of $X$, we find a common refinement $\mathcal{U}^{\prime \prime}$ of both. Again, by Lemma 4.10, one constructs an isomorphism

$$
\nu_{\mathcal{U}, \mathcal{U}^{\prime \prime}}:(\tilde{H}, \tilde{\nabla})_{\mathcal{U}} \cong(\tilde{H}, \tilde{\nabla})_{\mathcal{U}^{\prime \prime}}
$$

and similarly $\nu_{\mathcal{U}^{\prime}, \mathcal{U}^{\prime \prime}}$. Then define the isomorphism

$$
\nu_{\mathcal{U}, \mathcal{U}^{\prime}}=\nu_{\mathcal{U}^{\prime}, \mathcal{U}^{\prime \prime}}^{-1} \circ \nu_{\mathcal{U}, \mathcal{U}^{\prime \prime}}:(\tilde{H}, \tilde{\nabla})_{\mathcal{U}} \cong(\tilde{H}, \tilde{\nabla})_{\mathcal{U}^{\prime}}
$$

which satisfies also the cocycle relation for any three choices of open affine coverings (another way to remove this independence is to take the direct limit with respect to the directed set of all open affine coverings with the partial order given by refinement). Thus, we get our functor $\mathcal{T}_{n}$ by associating $(\tilde{H}, \tilde{\nabla})$ to $(E, \bar{H})$ as above which is defined up to canonical isomorphism.

\section{Second approach.}

This approach is due to the referee, who gave us a global construction of the functor from the whole category $\mathcal{H}(X)$ to $M I C^{(-1)}(X)$ in the weight one case. We shall generalize his/her method in the following.

Notation as above. For an object $(E, \theta, \bar{H}, \bar{\nabla}, \overline{F i l}, \bar{\psi}) \in \mathcal{H}(X)$, one notices that the isomorphism of graded Higgs modules $\bar{\psi}: G r_{\overline{F i l}}(\bar{H}, \bar{\nabla}) \cong(\bar{E}, \bar{\theta})$ allows us to define the following composite morphism:

$$
j: \overline{F i l}^{i} \rightarrow \overline{F i l}^{i} / \overline{F i l}^{i+1} \stackrel{\bar{\psi}}{\cong} \bar{E}^{i} .
$$

Let $\eta: E^{i} \rightarrow \bar{E}^{i}$ be the natural projection by the reduction modulo $p^{n-1}$. Let $\overline{F i l}^{i} \times_{\bar{E}^{i}} E^{i}$ denote the kernel of the morphism of $\mathcal{O}_{X^{-}}$modules:

$$
\overline{F i l}^{i} \oplus E^{i} \stackrel{-j+\eta}{\longrightarrow} \bar{E}^{i} .
$$

We are going to associate to $(E, \bar{H})$ a twisted flat module $\left(H^{\sharp}, \nabla^{\sharp}\right)$. The $\mathcal{O}_{X^{-}}$ module $H^{\sharp}$ is defined to be the cokernel of the morphism

$$
\oplus_{i}\left(\overline{F i l}^{i} \times_{\bar{E}^{i}} E^{i}\right) \stackrel{\epsilon}{\longrightarrow} \oplus_{i}\left(\overline{F i l}^{i} \times_{\bar{E}^{i}} E^{i}\right),
$$

where for $x \times y \in \overline{F i l}^{i+1} \times_{\bar{E}^{i+1}} E^{i+1}$,

$$
\epsilon(x \times y)=(x \times 0)+(-p x \times-p y) \in\left(\overline{F i l}^{i} \times_{\bar{E}^{i}} E^{i}\right) \oplus\left(\overline{F i l}^{i+1} \times_{\bar{E}^{i+1}} E^{i+1}\right) .
$$

Let $c: \oplus_{i}\left(\overline{F i l}^{i} \times_{\bar{E}^{i}} E^{i}\right) \rightarrow H^{\sharp}$ be the natural morphism. We define a $W_{n^{-}}$-linear additive map (not a connection) as follows:

$$
\nabla^{\prime \prime}: \oplus_{i}\left(\overline{F i l}^{i} \times_{\bar{E}^{i}} E^{i}\right) \rightarrow \oplus_{i}\left(\overline{F i l}^{i-1} \times_{\bar{E}^{i-1}} E^{i-1}\right) \otimes \Omega_{X},
$$

which takes $x \times y \in \overline{F i l}^{i} \times_{\bar{E}^{i}} E^{i}$ to $\bar{\nabla}(x) \times \theta(y) \in\left(\overline{F i l}^{i-1} \times_{\bar{E}^{i-1}} E^{i-1}\right) \otimes \Omega_{X}$. Here we have used the natural identification

$$
\left(\overline{F i l}^{i-1} \otimes \Omega_{\bar{X}}\right) \times_{\left(\bar{E}^{i-1} \otimes \Omega_{\bar{X}}\right)}\left(E^{i-1} \otimes \Omega_{X}\right) \cong\left(\overline{F i l}^{i-1} \times_{\bar{E}^{i-1}} E^{i-1}\right) \otimes \Omega_{X} .
$$

We shall show that this map descends to a $p$-connection over $H^{\sharp}$. 
Lemma 4.12. Let $A_{i}$ be the image of $\overline{F i l}^{i} \times_{\bar{E}^{i}} E^{i}$ under the map $\epsilon$. Then

$$
\nabla^{\prime \prime}\left(A_{i}\right) \subset A_{i-1} \otimes \Omega_{X}
$$

and the induced $W_{n}$-linear additive map $\nabla^{\sharp}: H^{\sharp} \rightarrow H^{\sharp} \otimes \Omega_{X}$ is an integrable p-connection.

Proof. For $x \times y \in \overline{F i l}^{i} \times_{\bar{E}^{i}} E^{i}$, one computes that

$$
\nabla^{\prime \prime}(\epsilon(x \times y))=\bar{\nabla}(x) \times 0+\bar{\nabla}(-p x) \times \theta(-p y)=\epsilon(\bar{\nabla}(x) \times \theta(y)),
$$

which lies in $A_{i-1} \otimes \Omega_{X}$. Therefore, $\nabla^{\prime \prime}$ induces an additive $W_{n}$-linear map $\nabla^{\sharp}$ on $H^{\sharp}$. It suffices to show $\nabla^{\sharp}$ is a $p$-connection, as the integrability follows directly from the integrabilities of $\bar{\nabla}$ and $\theta$. For $f \in \mathcal{O}_{X}$ and $x \times y \in \overline{F i l}^{i} \times_{\bar{E}^{i}} E^{i}$, it is reduced to show the equality

$$
\nabla^{\sharp}(f c(x \times y))=f \nabla^{\sharp}(c(x \times y))+p c(x \times y) \otimes d f .
$$

Because

$$
\begin{aligned}
\nabla^{\prime \prime}(f(x \times y)) & =\nabla^{\prime \prime}(\bar{f} \cdot x \times f \cdot y)=\bar{\nabla}(\bar{f} \cdot x) \times \theta(f y) \\
& =(d \bar{f} \cdot x+\bar{f} \bar{\nabla}(x)) \times f \theta(y)=d \bar{f} \cdot x \times 0+\bar{f} \bar{\nabla}(x) \times f \theta(y) \\
& =(x \times 0) \otimes d f+f \nabla^{\prime \prime}(x \times y),
\end{aligned}
$$

where $\bar{f} \in \mathcal{O}_{\bar{X}}$ is the $\bmod p^{n-1}$-reduction of $f$, and because $c(x \times 0)=c(p(x \times y))$, the required equality follows.

Lemma 4.13. Notation as above. The p-connection $\nabla^{\sharp}$ extends to a twisted connection.

Proof. This step is similar to Lemma 4.8. Let $D_{1}, \cdots, D_{p-1+m} \in T_{X}$ for $m \geq 0$. Note that

$$
\nabla_{D_{m+1}}^{\prime \prime} \circ \cdots \circ \nabla_{D_{p-1+m}}^{\prime \prime}: \overline{F i l}^{i} \times_{\bar{E}^{i}} E^{i} \rightarrow \overline{F i l}^{i+1-p} \times_{\bar{E}^{i+1-p}} E^{i+1-p},
$$

and as $\overline{F i l}^{i}=0, E^{i}=0$ for $i \geq w+1$ and $w \leq p-2$, the image of $\nabla_{D_{m+1}}^{\prime \prime} \circ \cdots \circ$ $\nabla_{D_{p-1+m}}^{\prime \prime}$ lies in $\overline{F i l}^{i} \times_{\bar{E}^{i}} E^{i}$ for $i \leq-1$. As $\overline{F i l}^{i}=\bar{H}$ and $\bar{E}^{i}=E^{i}=0$ for $i \leq-1$, one defines the map

$$
\frac{\nabla^{\prime \prime}}{p}:=\bar{\nabla} \times 0: \overline{F i l}^{i} \times_{\bar{E}^{i}} E^{i} \rightarrow \overline{F i l}^{i} \times_{\bar{E}^{i}} E^{i} \otimes \Omega_{X}, \quad i \leq-1 .
$$

Then the map

$$
\nabla^{\sharp}\left(\gamma_{m}\left(D_{1}, \cdots, D_{p-1+m}\right)\right):=\frac{\nabla_{D_{1}}^{\prime \prime}}{p} \circ \cdots \frac{\nabla_{D_{m}}^{\prime \prime}}{p} \circ \nabla_{D_{m+1}}^{\prime \prime} \circ \cdots \circ \nabla_{D_{p-1+m}}^{\prime \prime}
$$

gives rise to a twisted connection extending the $p$-connection $\nabla^{\sharp}$.

Equivalence.

Proposition 4.14. The functor $\mathcal{T}_{n}^{\sharp}$, restricted to the subcategory $\mathcal{H}_{l f}(X)$, is naturally equivalent to the functor $\mathcal{T}_{n}$. 
Proof. Given an object $(E, \bar{H}) \in \mathcal{H}_{l f}(X)$, we set

$$
(\tilde{H}, \tilde{\nabla}):=\mathcal{T}_{n}(E, \bar{H}), \quad\left(H^{\sharp}, \nabla^{\sharp}\right):=\mathcal{T}_{n}^{\sharp}(E, \bar{H})
$$

defined as above. We are going to exhibit a natural isomorphism

$$
\lambda:\left(H^{\sharp}, \nabla^{\sharp}\right) \cong(\tilde{H}, \tilde{\nabla}) .
$$

Without loss of generality, we may assume that there is an object $(H, \nabla, F i l) \in$ $M C F_{p-2}$ such that $R_{n}(H, \nabla, F i l)=(E, \bar{H})$ and $G_{n}(H, \nabla, F i l)=(\tilde{H}, \widetilde{\nabla})$. Consider the following commutative diagram:

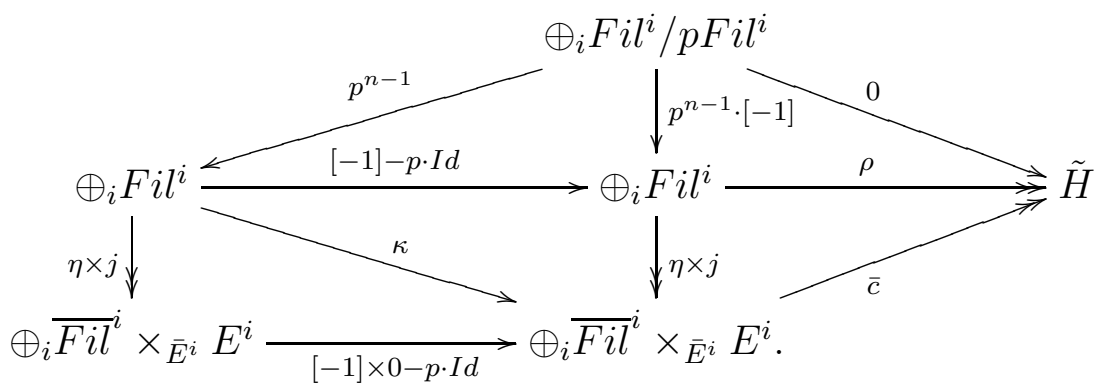

The middle row is (4.5.1), the exact sequence defining $\tilde{H}$. The middle column is also an exact sequence because of locally free assumption, where the map $\eta$ denotes the reduction map by mod $p^{n-1}$ and $j$ the natural projection $F i l^{i} \rightarrow$ $F i l^{i} / F i l^{i+1}=E^{i}$. By the commutativity of left upper triangle, we see that the map $\rho$ factors through the surjective map $\bar{c}$, so that there is an exact sequence

$$
\oplus_{i} F i l^{i} \stackrel{\kappa}{\longrightarrow} \oplus_{i} \overline{F i l}^{i} \times_{\bar{E}^{i}} E^{i} \stackrel{\bar{c}}{\longrightarrow} \tilde{H} \longrightarrow 0 .
$$

By the commutativity of the left lower triangle and the surjectivity of the left vertical map $\eta \times j$, the above exact sequence is replaced by the following exact sequence:

$$
\oplus_{i} \overline{F i l}^{i} \times_{\bar{E}^{i}} E^{i} \stackrel{[-1] \times 0-p \cdot I d}{\longrightarrow} \oplus_{i} \overline{F i l}^{i} \times_{\bar{E}^{i}} E^{i} \stackrel{\bar{c}}{\longrightarrow} \tilde{H} \longrightarrow 0 .
$$

Thus there is a unique isomorphism $\lambda: H^{\sharp} \rightarrow \tilde{H}$, to render the following diagram commutative

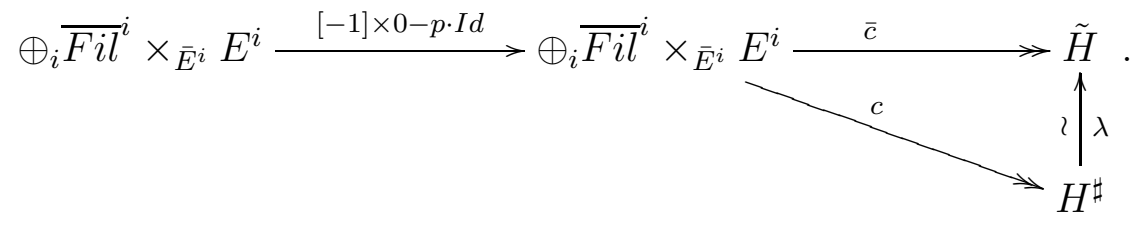

The isomorphism $\lambda$ is compatible with twisted connections. Indeed, the twisted connection $\tilde{\nabla}$ on $\tilde{H}$ is induced by the maps

$$
\nabla^{\prime}: F i l^{i} \rightarrow F i l^{i-1} \otimes \Omega_{X_{n}}, \quad i \geq 0,
$$

and

$$
\frac{\nabla^{\prime}}{p}: F i l^{j} \rightarrow F i l^{j} \otimes \Omega_{X_{n}}, \quad j \leq-1,
$$

while $\nabla^{\sharp}$ on $H^{\sharp}$ by the formula (4.11.1) and $\frac{\nabla^{\prime \prime}}{p}$ in Lemma 4.13. Since the vertical map $\eta \times j$ in the middle column of diagram (4.14.1) is compatible with the map 
$\nabla^{\prime}$ on $\oplus_{i} F i l^{i}$ and the map $\nabla^{\prime \prime}$ on $\oplus_{i} \overline{F i l}^{i} \times_{\bar{E}^{i}} E^{i}$, and it is also compatible with the map $\frac{\nabla^{\prime}}{p}$ on $\oplus_{j \leq-1} F i l^{j}$ and the map $\frac{\nabla^{\prime \prime}}{p}$ on $\oplus_{j \leq-1} \overline{F i l}^{j} \times_{\bar{E}^{j}} E^{j}$, it follows that the map $\lambda$ is compatible with $\widetilde{\nabla}$ on $\tilde{H}$ and $\nabla^{\sharp}$ on $H^{\sharp}$.

This completes the construction of the first functor from $\mathcal{H}\left(X_{n}^{\prime}\right)$ to $\widetilde{M I C}\left(X_{n}^{\prime}\right)$, by setting $X=X_{n}^{\prime}$ in the above two approaches.

Next, we are going to construct the second functor $\mathcal{F}_{n}: \widetilde{M I C}\left(X_{n}^{\prime}\right) \rightarrow \operatorname{MIC}\left(X_{n}\right)$. In contrast to the first functor, it requires the $W_{n+1}$-liftability of $X_{n}^{\prime}$ and depends on the choice of $W_{n+1}$-liftings. Again, the method of "local lifting-global gluing" in the first approach of the former functor will be applied. Here "local lifting" means a local lifting of the relative Frobenius and the gluing morphisms are provided by the difference of two relative Frobenius liftings via the Taylor formula. We shall remind that A. Shiho [29] has obtained the functor from the category of quasi-nilpotent modules with integrable $p$-connections over $X_{n}^{\prime}$ to the category of flat modules over $X_{n}$ under the assumptions both on the existence of $W_{n+1^{-}}$ liftings of $X_{n}^{\prime}, X_{n}$ and on the existence of the relative Frobenius lifting over $W_{n+1}$. His construction is closely related to ours in the local case, but they were independently obtained.

Proposition 4.15. Let $X$ be a smooth scheme over $S_{n}$ and $X^{\prime}=X \times_{F_{S_{n}}} S_{n}$. Assume $X^{\prime}$ admits a smooth lifting $\tilde{X}^{\prime}$ over $S_{n+1}$. Then there is a functor $\mathcal{F}_{n}$ from the category of $\widehat{M I C}\left(X^{\prime}\right)$ to the category $M I C(X)$.

Proof. We divide the whole construction into several small steps.

Step 0. For convenience, let us introduce $\tilde{X}=\tilde{X}^{\prime} \times_{F_{S_{n+1}}^{-1}} S_{n+1}$, which lifts $X$ and will be used in Step 2. Let $\tilde{\mathcal{U}}^{\prime}=\left\{\tilde{U}_{i}^{\prime}\right\}_{i \in I}$ be an open affine covering of $\tilde{X}^{\prime}$ (assume each member is flat over $S_{n+1}$ ). Then by the obvious base change, it induces an open affine covering $\tilde{\mathcal{U}}=\left\{\tilde{U}_{i}\right\}$ of $\tilde{X}$, and by reduction modulo $p^{n}$, open affine coverings $\mathcal{U}^{\prime}(\operatorname{resp} . \mathcal{U})$ of $X^{\prime}($ resp. $X)$. For each $i \in I$, we take a morphism $\tilde{F}_{i}: \tilde{U}_{i} \rightarrow \tilde{U}_{i}^{\prime}$ over $S_{n+1}$ lifting the relative Frobenius $\tilde{U} \otimes \mathbb{Z} / p \mathbb{Z} \rightarrow \tilde{U}^{\prime} \otimes \mathbb{Z} / p \mathbb{Z}$ over $k$ and denote $\tilde{F}_{i} \otimes \mathbb{Z} / p^{n} \mathbb{Z}$ by $F_{i}$. Given a twisted flat module $(\tilde{H}, \tilde{\nabla})$ over $X^{\prime}$, its restriction to $U_{i}^{\prime}$ is denoted by $\left(\tilde{H}_{i}, \tilde{\nabla}_{i}\right)$.

Step 1. Each $\tilde{F}_{i}$ defines a morphism over $S_{n}$ :

$$
\frac{d \tilde{F}_{i}}{p}: F_{i}^{*} \Omega_{U_{i}^{\prime}} \rightarrow \Omega_{U_{i}}
$$

Put $H_{i}:=F_{i}^{*} \tilde{H}_{i}$. As discussed before (see the paragraph after Definition 4.5), one can naturally regard $\tilde{\nabla}_{i}$ as an integrable $p$-connection on $\tilde{H}_{i}$. By doing so, we can consider the following formula:

$$
\nabla_{i}(f \otimes e):=d f \otimes e+f \cdot\left(\frac{d \tilde{F}_{i}}{p} \otimes 1\right)\left(1 \otimes \tilde{\nabla}_{i}(e)\right), \quad f \in \mathcal{O}_{U_{i}}, \quad e \in \tilde{H}_{i} .
$$

Claim 4.16. $\nabla_{i}$ is well-defined and it defines an integrable connection on $H_{i}$. 
Proof. One computes that

$$
\begin{aligned}
\nabla_{i}(1 \otimes f e) & =\left(\frac{d \tilde{F}_{i}}{p} \otimes 1\right)\left(1 \otimes \tilde{\nabla}_{i}(f e)\right)=\left(\frac{d \tilde{F}_{i}}{p} \otimes 1\right)\left(1 \otimes p d f \cdot e+1 \otimes f \cdot \tilde{\nabla}_{i}(e)\right) \\
& =d\left(F_{i}^{*} f\right) \otimes e+F_{i}^{*} f \cdot\left(\frac{d \tilde{F}_{i}}{p} \otimes 1\right)\left(1 \otimes \tilde{\nabla}_{i}(e)\right)=\nabla_{i}\left(F_{i}^{*} f \otimes e\right),
\end{aligned}
$$

which shows the well-definedness. Let $\left\{t_{1}, \cdots t_{d}\right\}$ be a set of étale local coordinates of $U_{i}$ and $\left\{t_{\alpha}^{\prime}\right\}_{1 \leq \alpha \leq d}$ the corresponding one of $U_{i}^{\prime}$. Note for $1 \leq j, k \leq d$ and any $e \in \tilde{H}_{i}$,

$$
\nabla_{i}\left(\partial t_{j}\right)(1 \otimes e)=\sum_{\alpha=1}^{d}\left(\partial t_{j} \frac{d \tilde{F}_{i}}{p}\left(1 \otimes d t_{\alpha}^{\prime}\right)\right) \otimes \tilde{\nabla}\left(\partial t_{\alpha}^{\prime}\right)(e) .
$$

So one computes that

$$
\begin{gathered}
\nabla_{i}\left(\partial t_{k}\right) \circ \nabla_{i}\left(\partial t_{j}\right)(1 \otimes e)=\sum_{\alpha=1}^{d} \partial_{t_{k}}\left(\partial t_{j} \frac{d \tilde{F}_{i}}{p}\left(1 \otimes d t_{\alpha}^{\prime}\right)\right) \otimes \tilde{\nabla}\left(\partial t_{\alpha}^{\prime}\right)(e) \\
+\sum_{1 \leq \alpha, \beta \leq d}\left(\partial t_{j} \frac{d \tilde{F}_{i}}{p}\left(1 \otimes d t_{\alpha}^{\prime}\right)\right) \cdot\left(\partial t_{k} \frac{d \tilde{F}_{i}}{p}\left(1 \otimes d t_{\beta}^{\prime}\right)\right) \otimes \tilde{\nabla}\left(\partial t_{\beta}^{\prime}\right) \circ \tilde{\nabla}\left(\partial t_{\alpha}^{\prime}\right)(e) \\
=\sum_{\alpha=1}^{d} \partial_{t_{j}}\left(\partial t_{k} \frac{d \tilde{F}_{i}}{p}\left(1 \otimes d t_{\alpha}^{\prime}\right)\right) \otimes \tilde{\nabla}\left(\partial t_{\alpha}^{\prime}\right)(e) \\
+\sum_{1 \leq \alpha, \beta \leq d}\left(\partial t_{j} \frac{d \tilde{F}_{i}}{p}\left(1 \otimes d t_{\alpha}^{\prime}\right)\right) \cdot\left(\partial t_{k} \frac{d \tilde{F}_{i}}{p}\left(1 \otimes d t_{\beta}^{\prime}\right)\right) \otimes \tilde{\nabla}\left(\partial t_{\alpha}^{\prime}\right) \circ \tilde{\nabla}\left(\partial t_{\beta}^{\prime}\right)(e) \\
=\nabla_{i}\left(\partial t_{j}\right) \circ \nabla_{i}\left(\partial t_{k}\right)(1 \otimes e) .
\end{gathered}
$$

In the above second equality we have used the integrability of $\tilde{\nabla}_{i}$, i.e., $\tilde{\nabla}_{i}\left(\partial t_{\alpha}^{\prime}\right)$ commutes with $\tilde{\nabla}_{i}\left(\partial t_{\beta}^{\prime}\right)$. Then for any $f \in \mathcal{O}_{U_{i}}$ and any $e \in \tilde{H}_{i}$, one has

$$
\begin{gathered}
\nabla_{i}\left(\partial t_{k}\right) \circ \nabla_{i}\left(\partial t_{j}\right)(f \otimes e) \\
=\frac{\partial^{2} f}{\partial t_{k} \partial t_{j}} \otimes e+\frac{\partial f}{\partial t_{j}} \cdot \nabla_{i}\left(\partial t_{k}\right)(1 \otimes e)+\frac{\partial f}{\partial t_{k}} \cdot \nabla_{i}\left(\partial t_{j}\right)(1 \otimes e)+f \cdot \nabla_{i}\left(\partial t_{k}\right) \nabla_{i}\left(\partial t_{j}\right)(1 \otimes e) \\
=\frac{\partial^{2} f}{\partial t_{j} \partial t_{k}} \otimes e+\frac{\partial f}{\partial t_{j}} \cdot \nabla_{i}\left(\partial t_{k}\right)(1 \otimes e)+\frac{\partial f}{\partial t_{k}} \cdot \nabla_{i}\left(\partial t_{j}\right)(1 \otimes e)+f \cdot \nabla_{i}\left(\partial t_{j}\right) \nabla_{i}\left(\partial t_{k}\right)(1 \otimes e) \\
=\nabla_{i}\left(\partial t_{j}\right) \circ \nabla_{i}\left(\partial t_{k}\right)(f \otimes e) .
\end{gathered}
$$

So $\nabla_{i}\left(\partial t_{k}\right)$ commutes with $\nabla_{i}\left(\partial t_{j}\right)$ for $1 \leq k, j \leq d$, that is, $\nabla_{i}$ is integrable as claimed.

Step 2. The previous step provides us a set of local flat modules $\left\{\left(H_{i}, \nabla_{i}\right)\right\}_{i \in I}$ and we want to glue them into one global flat module. The point is to use the Taylor formula involving the difference of two relative Frobenius liftings to construct the gluing morphisms. This is well-known if $p$ is non-nilpotent in the base ring, and we use the formalism of a twisted connection to make sense of the $p$-powers in the denominators of the Taylor formula.

Let $\tilde{U}_{i}=\operatorname{Spec} \tilde{R}_{i}, i=1,2$ be two smooth schemes over $S_{n+1}$, equipped with a lifting of relative Frobenius $\tilde{F}_{i}: \tilde{U}_{i} \rightarrow \tilde{U}_{i}^{\prime}$. Assume there is a set of étale local coordinates $\left\{\tilde{t}_{i}\right\}$ for $\tilde{U}_{1}$ and suppose there is a morphism $\iota: \tilde{U}_{2} \rightarrow \tilde{U}_{1}$ over $S_{n+1}$, which induces the morphism $\iota^{\prime}: \tilde{U}_{2}^{\prime} \rightarrow \tilde{U}_{1}^{\prime}$. Let $R_{i}=\tilde{R}_{i} \otimes \mathbb{Z} / p^{n} \mathbb{Z}, U_{i}=\operatorname{Spec} R_{i}$ and $F_{i}=\tilde{F}_{i} \otimes \mathbb{Z} / p^{n} \mathbb{Z}$. Let $\left\{\tilde{t}_{i}^{\prime}\right\}$ (resp. $\left\{t_{i}\right\}$ ) be the induced coordinate functions on $\tilde{U}_{1}^{\prime}$ 
(resp. $\left.U_{1}\right)$. Given a twisted flat module $(\tilde{H}, \tilde{\nabla})$ over $U_{1}^{\prime}$, there is an isomorphism of $R_{2}$-modules $G_{21}: F_{2}^{*} \iota^{\prime *} \tilde{H} \cong \iota^{*} F_{1}^{*} \tilde{H}$ given by the Taylor formula

$$
G_{21}(e \otimes 1)=\sum_{J} \frac{\tilde{\nabla}(\partial)^{J}}{J !}(e) \otimes z^{J}, e \in \tilde{H},
$$

where $J$ is a multi-index $J:=\left(j_{1}, \cdots, j_{d}\right)$ with each component $j_{l} \geq 0, J !:=$ $\prod_{l=1}^{d} j_{l}$ !, and

$$
\frac{\tilde{\nabla}(\partial)^{J}}{J !}:=\frac{\left(\tilde{\nabla}\left(\partial t_{1}^{\prime}\right)\right)^{j_{1}}}{j_{1} !} \circ \cdots \circ \frac{\left(\tilde{\nabla}\left(\partial t_{d}^{\prime}\right)\right)^{j_{d}}}{j_{d} !}
$$

with

$$
z^{J}:=\prod_{l=1}^{d} z_{l}^{j_{l}}, \quad z_{l}:=\frac{\left(\tilde{F}_{2}^{*} \iota^{* *}\right)\left(\tilde{t}_{l}^{\prime}\right)-\left(\iota^{*} \tilde{F}_{1}^{*}\right)\left(\tilde{t}_{l}^{\prime}\right)}{p} .
$$

As already explained right after Definition 4.5, $\frac{\tilde{\nabla}(\partial)^{J}}{J !}$ converges to zero $p$-adically as $|J| \rightarrow \infty$, the summation in the formula (4.16.1) is actually a finite sum. There is the cocycle relation between the isomorphisms for three objects, namely the equality $G_{31}=G_{21} \circ G_{32}$ holds. The proof is mostly formal: suppressing $\iota \mathrm{s}$ in the Taylor formula (4.16.1) and writing $\hat{z}$ (resp. $\tilde{z}$ ) for the $z$-function appeared in $G_{32}\left(\operatorname{resp} . G_{31}\right)$. Then as $\tilde{z}=z+\hat{z}$, one calculates that

$$
\begin{aligned}
G_{21} \circ G_{32}(e \otimes 1) & =\sum_{I} \sum_{J} \frac{\tilde{\nabla}(\partial)^{I+J}}{I ! J !}(e) \otimes z^{J} \cdot \hat{z}^{I} \\
& =\sum_{K} \frac{\tilde{\nabla}(\partial)^{K}}{K !}(e) \otimes\left(\sum_{I+J=K} \frac{K !}{I ! J !} \cdot z^{J} \cdot \hat{z}^{I}\right) \\
& =\sum_{K} \frac{\tilde{\nabla}(\partial)^{K}}{K !}(e) \otimes(z+\hat{z})^{K} \\
& =\sum_{K} \frac{\tilde{\nabla}(\partial)^{K}}{K !}(e) \otimes \tilde{z}^{K} \\
& =G_{31}(e \otimes 1) .
\end{aligned}
$$

The cocycle condition follows. Returning to our case, we shall apply the Taylor formula in the following way: write $\tilde{U}_{i j}=\tilde{U}_{i} \cap \tilde{U}_{j}$ and take a relative Frobenius lifting $\tilde{F}_{i j}: \tilde{U}_{i j} \rightarrow \tilde{U}_{i j}^{\prime}$. Let $\iota_{1}: \tilde{U}_{i j} \rightarrow \tilde{U}_{i}$ and $\iota_{2}: \tilde{U}_{i j} \rightarrow \tilde{U}_{j}$ be the natural inclusions. Then we obtain the isomorphisms $\alpha_{i}$ and $\alpha_{j}$ in the following diagram:

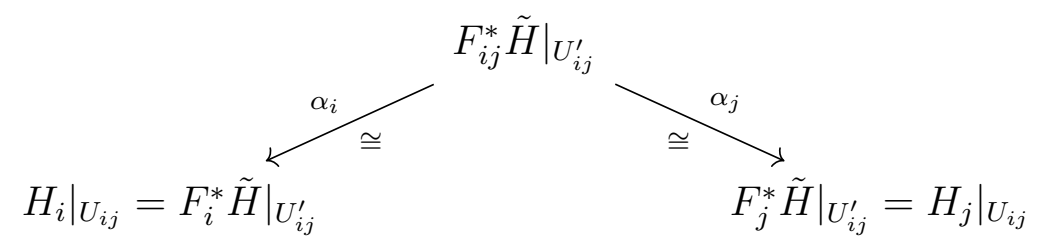

The we define the isomorphism $G_{i j}:=\alpha_{j} \circ \alpha_{i}^{-1}:\left.\left.H_{i}\right|_{U_{i j}} \rightarrow H_{j}\right|_{U_{i j}}$ and the set of isomorphisms $\left\{G_{i j}\right\}$ satisfies the cocycle condition. There is one more property of $G_{i j}$, namely, it is compatible with connections. For $1 \leq l \leq d$, let us identify 
$l$ with the multiple index $(0, \cdots, 1, \cdots, 0)$ with 1 at the $l$-th position. Then one calculates that

$$
\left(I d \otimes G_{i j}\right)\left(\nabla_{i}(e \otimes 1)\right)=\sum_{J} \sum_{l=1}^{d} \frac{\tilde{\nabla}(\partial)^{J+l}}{J !}(e) \otimes z^{J} \cdot \frac{d \tilde{F}_{i}}{p}\left(d t_{l}^{\prime}\right),
$$

and

$$
\nabla_{j}\left(G_{i j}(e \otimes 1)\right)=\sum_{J} \sum_{l=1}^{d} \frac{\tilde{\nabla}(\partial)^{J+l}}{J !}(e) \otimes z^{J} \cdot\left(\frac{d \tilde{F}_{j}}{p}\left(d t_{l}^{\prime}\right)+d z_{l}\right)
$$

As

$$
d z_{l}=\frac{d \tilde{F}_{i}}{p}\left(d t_{l}^{\prime}\right)-\frac{d \tilde{F}_{j}}{p}\left(d t_{l}^{\prime}\right)
$$

it follows that

$$
\left(I d \otimes G_{i j}\right)\left(\nabla_{i}(e \otimes 1)\right)=\nabla_{j}\left(G_{i j}(e \otimes 1)\right) .
$$

Thus, we use the set $\left\{G_{i j}\right\}$ to glue the local flat modules $\left\{\left(H_{i}, \nabla_{i}\right)\right\}$ together and obtain an object $(H, \nabla)$ in $M I C(X)$.

Step 3. We need to show the so-constructed object $(H, \nabla)$ is independent of the choice of relative Frobenius liftings and the choice of open affine coverings. But, after our argument on a similar independence-type statement in Proposition 4.11, this step becomes entirely formal. Therefore, to summarize the whole steps, we construct a flat module $(H, \nabla)$ over $X$ up to canonical isomorphism from any twisted flat module $(\tilde{H}, \tilde{\nabla})$ over $X^{\prime}$, and this gives our second functor $\mathcal{F}_{n}$.

Remark 4.17. In above, a simplification can be made when a global lifting $F_{\tilde{X}}$ of the relative Frobenius over $\tilde{X}$ (a $W_{n+1}$-lifting of $X$ ) exists. Set $F_{X}=F_{\tilde{X}} \otimes \mathbb{Z} / p^{n} \mathbb{Z}$. In this case, one has a globally defined morphism $\frac{d F_{\tilde{X}}}{p}: F_{X}^{*} \Omega_{X^{\prime}} \rightarrow \Omega_{X}$. Then $H=F_{X}^{*} \tilde{H}$ and $\nabla$ is define by Formula (4.15.1) in which various local $\frac{d \tilde{F}_{i}}{p} \mathrm{~s}$ are replaced simply by $\frac{d F_{\tilde{X}}}{p}$. The reason is as follows: in Step 0, we may take an open affine covering $\tilde{\mathcal{U}}=\left\{\tilde{U}_{i}\right\}$ of $\tilde{X}$ (whose element is flat over $W_{n+1}$ ) such that $F_{\tilde{X}}: \tilde{U}_{i} \rightarrow \tilde{U}_{i}^{\prime}$ for all $i$ and then take $\tilde{F}_{i}$ to be the restriction to $F_{\tilde{X}}$ to $\tilde{U}_{i}$. Then it follows that the gluing functions $G_{i j}$ s are all identity.

Now we proceed to the proof of Theorem 4.1 .

Proof of Theorem 4.1. We define the functor $\mathcal{C}_{n}^{-1}: \mathcal{H}\left(X_{n}^{\prime}\right) \rightarrow M I C\left(X_{n}\right)$ to be the composite of the functor $\mathcal{T}_{n}: \mathcal{H}\left(X_{n}^{\prime}\right) \rightarrow \widehat{M I C}\left(X_{n}^{\prime}\right)$ in Proposition 4.11 and the functor $\mathcal{F}_{n}: \widetilde{M I C}\left(X_{n}^{\prime}\right) \rightarrow M I C\left(X_{n}\right)$ in Proposition 4.15. From their very constructions, the two functors $\mathcal{T}_{n}$ and $\mathcal{F}_{n}$ are compatible with the reduction modulo $p^{n-1}$. So is their composite. The equivalence of the functor $\mathcal{C}_{1}^{-1}$ over $W_{1}=k$ with the inverse Cartier transform of Ogus-Vologodsky [25] has been verified in [13. 


\section{Higgs CORRESPONDENCE IN MiXED CHARACTERISTIC}

The section aims to establish the Higgs correspondence between the category of strict $p^{n}$-torsion Fontaine modules with endomorphism $W_{n}\left(\mathbb{F}_{p^{f}}\right)$ (Variant $2 \S 2$ ) and the category of periodic Higgs-de Rham flows over $X_{n}$, for $n \geq 2$, so that it lifts the one in positive characteristic and its limit, as $n$ goes to infinity, yields the Higgs correspondence in mixed characteristic.

We proceed first to the definition of a Higgs-de Rham flow over $X_{n}$. We use the notation from $\S 4$. Let $\pi_{n}: X_{n}^{\prime} \rightarrow X_{n}$ be the natural morphism by base change, which induces the obvious equivalence of categories $\pi_{n}^{*}: \mathcal{H}\left(X_{n}\right) \cong \mathcal{H}\left(X_{n}^{\prime}\right)$. We define the functor

$$
C_{n}^{-1}:=\mathcal{C}_{n}^{-1} \circ \pi_{n}^{*}: \mathcal{H}\left(X_{n}\right) \rightarrow \operatorname{MIC}\left(X_{n}\right) .
$$

Definition 5.1. A Higgs-de Rham flow over $X_{n}$ is a diagram of the following form:

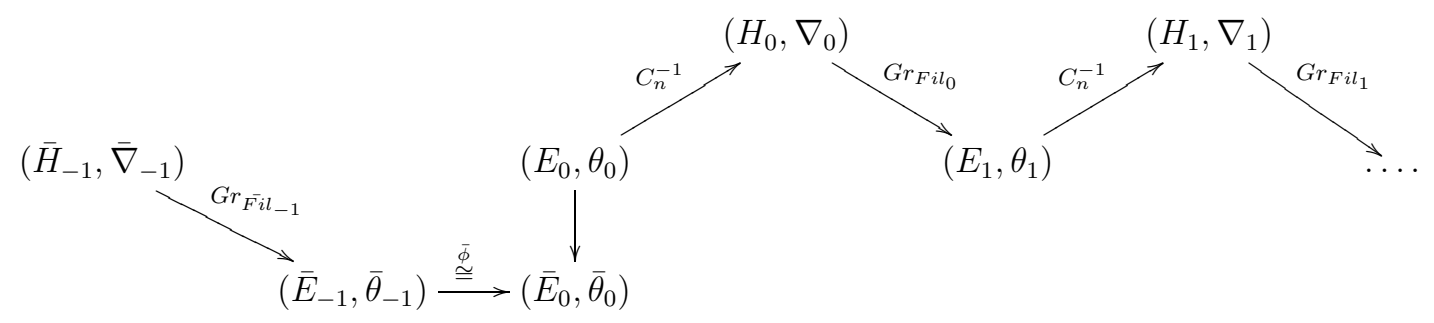

Here in the upper line $\left(H_{i}, \nabla_{i}\right):=C_{n}^{-1}\left(E_{i}, \theta_{i}\right)$ and $F i l_{i}$ is a finite exhaustive decreasing filtration of $\mathcal{O}_{X_{n}}$-submodules on $H_{i}$ satisfying Griffiths' transversality with respect to $\nabla_{i}$; in the middle line each term $\left(E_{i}, \theta_{i}\right)$ is a graded Higgs module over $X_{n}$ and $\left(\bar{H}_{-1}, \bar{\nabla}_{-1}, \overline{F i} l_{-1}\right)$ is a de Rham module over $X_{n-1}$; in the bottom line $\bar{\phi}$ is an isomorphism of graded Higgs modules from $\left(\bar{E}_{-1}, \bar{\theta}_{-1}\right)=$ $G r_{\bar{F}^{i} l_{-1}}\left(\bar{H}_{-1}, \bar{\nabla}_{-1}\right)$ to $\left(\bar{E}_{0}, \bar{\theta}_{0}\right)$, the $\bmod p^{n-1}$ reduction of $\left(E_{0}, \theta_{0}\right)$.

We emphasize that, in the above definition, the filtrations $F i l_{i}, i \geq 0$ and the isomorphism $\bar{\phi}$ are part of the defining data of the flow. Also, an explanation of the various inverse Cartier transforms in the flow is in order: $C_{n}^{-1}\left(E_{0}, \theta_{0}\right)$ is the abbreviation for

$$
C_{n}^{-1}\left(E_{0}, \theta_{0}, \bar{H}_{-1}, \bar{\nabla}_{-1}, \overline{F i l}_{-1}, \bar{\phi}\right),
$$

and $C_{n}^{-1}\left(E_{i}, \theta_{i}\right)$ for $i \geq 1$ is the abbreviation for

$$
\left.C_{n}^{-1}\left(E_{i}, \theta_{i},\left(C_{n}^{-1}\left(E_{i-1}, \theta_{i-1}\right), F i l_{i-1}\right) \otimes \mathbb{Z} / p^{n-1} \mathbb{Z}\right), I d\right) .
$$

Comparing with the notion of a periodic Higgs-de Rham flow over $X_{1}$ (Definition 3.1), one finds that the extra data in the lower left corner in Definition 5.1puts an additional condition on $\left(E_{0}, \theta_{0}\right)$ when $n \geq 2$. This is caused by the construction of the functor $C_{n}^{-1}, n \geq 2$. It is interesting to characterize those graded Higgs modules satisfying the condition, but more importantly, to know whether this assumption made on $C_{n}^{-1}, n \geq 2$ could be relaxed after all.

Next, we turn to the central notion of a periodic Higgs-de Rham over $X_{n}$ which is defined in an inductive way. For a flat bundle $(H, \nabla)$ over $X_{n}, n \geq 1$, a finite 
exhaustive decreasing filtration on $H$ is said to be a Hodge filtration, if it consists of locally free subsheaves of $H$, is locally split, and obeys Griffiths' transversality with respect to $\nabla$.

Definition 5.2. A periodic Higgs-de Rham flow over $X_{n}, n \geq 2$ of period $f \in \mathbb{N}$ consists of the following data:

(1) A periodic Higgs-de Rham flow $\left(\bar{E}, \bar{\theta}, \bar{F} i l_{0}, \cdots, \overline{F i} l_{f-1}, \bar{\phi}\right) \in H D F_{p-2, f}\left(X_{n} / W_{n}\right)$;

(2) A graded Higgs bundle $(E, \theta) \in H I G_{p-2}\left(X_{n}\right)$ lifting $(\bar{E}, \bar{\theta})$;

(3) A Hodge filtration $F i l_{i}$ of level $\leq p-2$ on $C_{n}^{-1}\left(E_{i}, \theta_{i}\right)$ lifting the Hodge filtration $\bar{F}_{i} l_{i}$ on $C_{n-1}^{-1}\left(\bar{E}_{i}, \bar{\theta}_{i}\right)$;

(4) An isomorphism of graded Higgs modules over $X_{n}$

$$
\phi: \operatorname{Gr}_{F i l_{f-1}}\left(C_{n}^{-1}\left(E_{f-1}, \theta_{f-1}\right)\right) \cong(E, \theta)
$$

lifting $\bar{\phi}$.

In the above definition, $\left(E_{0}, \theta_{0}\right):=(E, \theta)$ and $\left(C_{n-1}^{-1}\left(\bar{E}_{f-1}, \bar{\theta}_{f-1}\right), \bar{F} i l_{f-1}, \bar{\phi}\right)$ together make an object in the category $\mathcal{H}\left(X_{n}\right)$ so that $C_{n}^{-1}\left(E_{0}, \theta_{0}\right)$ is naturally defined. Also, $C_{n}^{-1}\left(E_{i}, \theta_{i}\right)$ for $i \geq 1$ is naturally defined, which is simply

$$
C_{n}^{-1}\left(E_{i}, \theta_{i}, C_{n-1}^{-1}\left(E_{i-1}, \theta_{i-1}\right), \bar{F} i l_{i-1}\right) .
$$

By Corollary 3.14, the filtrations on a periodic Higgs-de Rham flow over $X_{1}$ are indeed Hodge filtrations.

Thus the data of a periodic Higgs-de Rham flow of period $f$ over $X_{n}, n \geq 2$ is encoded in the following tuple

$$
\left(E, \theta, F i l_{0}, \cdots, F i l_{f-1}, \phi, \bar{E}, \bar{\theta}, \bar{F} i l_{0}, \cdots, \bar{F} i l_{f-1}, \bar{\phi}\right) .
$$

A morphism between two tuples is given by a pair $(f, \bar{f})$, where $\bar{f}$ is a morphism in the category $H D F_{p-2, f}\left(X_{n} / W_{n}\right)$ and $f$ is a morphism of graded Higgs modules over $X_{n}$ lifting $\bar{f}$ and satisfying natural properties. Let us explain this in the one-periodic case in detail. Let $\left(E_{i}, \theta_{i}, F i l_{i}, \phi_{i}, \bar{E}_{i}, \bar{\theta}_{i}, \bar{F} i l_{i}, \bar{\phi}_{i}\right), i=1,2$ be two one-periodic flows over $X_{n}$. Then a morphism

$$
(f, \bar{f}):\left(E_{1}, \theta_{1}, F i l_{1}, \phi_{1}, \bar{E}_{1}, \bar{\theta}_{1}, \overline{F i} l_{1}, \bar{\phi}_{1}\right) \rightarrow\left(E_{2}, \theta_{2}, F i l_{2}, \phi_{2}, \bar{E}_{2}, \bar{\theta}_{2}, \bar{F} i l_{2}, \bar{\phi}_{2}\right)
$$

means the following: first,

$$
\bar{f}:\left(\bar{E}_{1}, \bar{\theta}_{1}, \overline{F i} l_{1}, \bar{\phi}_{1}\right) \rightarrow\left(\bar{E}_{2}, \bar{\theta}_{2}, \overline{F i} l_{2}, \bar{\phi}_{2}\right)
$$

is a morphism in $H D F_{p-2,1}\left(X_{n} / W_{n}\right)$; second, $f:\left(E_{1}, \theta_{1}\right) \rightarrow\left(E_{1}, \theta_{2}\right)$ is a morphism of graded Higgs modules over $X_{n}$ lifting

$$
\bar{f}:\left(\bar{E}_{1}, \bar{\theta}_{1}\right) \rightarrow\left(\bar{E}_{2}, \bar{\theta}_{2}\right) ;
$$

third, the morphism

$$
C_{n}^{-1}(f): C_{n}^{-1}\left(E_{1}, \theta_{1}\right) \rightarrow C_{n}^{-1}\left(E_{2}, \theta_{2}\right),
$$

which is naturally defined by the previous two properties, is compatible with the Hodge filtrations and the induced morphism on graded Higgs modules is 
compatible with $\phi$ s, that is, the following diagram commutes:

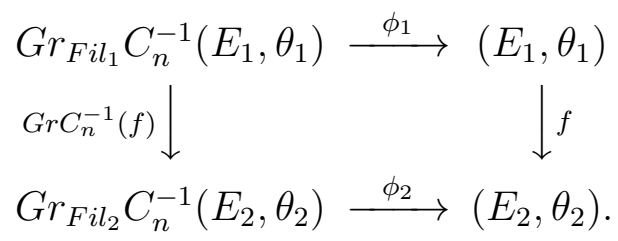

Thus, the category $H D F_{p-2, f}\left(X_{n+1} / W_{n+1}\right)$ of periodic Higgs-de Rham flow of period $f$ over $X_{n}$ is indeed inductively defined. Also, based upon the previous two definitions, it is straightforward to define a preperiodic Higgs-de Rham flow over $X_{n}$ (the key is to assure the well-definedness of the inverse Cartier transform of each Higgs term). Since this will be not used in the sequel, we leave this task to the reader. Recall that $M F_{[0, p-2], f}^{\nabla}\left(X_{n+1} / W_{n+1}\right)$ is the category of strict $p^{n}$-torsion Fontaine modules with extra endomorphism $W_{n}\left(\mathbb{F}_{p^{f}}\right)$. The following theorem lifts Theorem 3.2 in characteristic $p$ (but notice the stronger restriction on the Hodge-Tate weight).

Theorem 5.3. Let $X_{n+1}$ be a smooth scheme over $W_{n+1}$. For $f \in \mathbb{N}$, there is an equivalence of categories between the category $M F_{[0, p-2], f}^{\nabla}\left(X_{n+1} / W_{n+1}\right)$ and the category $H D F_{p-2, f}\left(X_{n+1} / W_{n+1}\right)$.

Similar to the characteristic $p$ case, the theorem will be reduced to the oneperiodic case. Let us introduce the category of one-periodic Higgs-de Rham flows over $X_{n}$ with endomorphism structure $W_{n}\left(\mathbb{F}_{p^{f}}\right)$. Its object is a tuple $(E, \theta, F i l, \phi, \iota, \bar{E}, \bar{\theta}, \overline{F i l}, \bar{\phi})$, where $(E, \theta, F i l, \phi, \bar{E}, \bar{\theta}, \bar{F} i l, \bar{\phi})$ is an object in the category $H D F_{n}:=H D F_{p-2, f}\left(X_{n+1} / W_{n+1}\right)$ and

$$
\iota: W_{n}\left(\mathbb{F}_{p^{f}}\right) \hookrightarrow \operatorname{End}_{H D F_{n}}(E, \theta, F i l, \phi, \bar{E}, \bar{\theta}, \bar{F} i l, \bar{\phi})
$$

is an embedding of $W_{n}\left(\mathbb{F}_{p}\right)$-algebras. A morphism of this category is a morphism of one-periodic Higgs-de Rham flows compatible with endomorphism structures. Thus Theorem 5.3 follows from the next two propositions.

Proposition 5.4. There is an equivalence of categories between the category $M F_{[0, p-2], 1}^{\nabla}\left(X_{n+1} / W_{n+1}\right)$ and the category $H D F_{p-2,1}\left(X_{n+1} / W_{n+1}\right)$.

This proposition is just the one-periodic case of Theorem 5.3, which implies immediately that $M F_{[0, p-2], f}^{\nabla}\left(X_{n+1} / W_{n+1}\right)$ is equivalent to the category of one-periodic Higgs-de Rham flows over $X_{n}$ with endomorphism structure $W_{n}\left(\mathbb{F}_{p^{f}}\right)$. We postpone its proof and show first the next

Proposition 5.5. There is an equivalence of categories between the category of one-periodic Higgs-de Rham flows over $X_{n}$ with endomorphism structure $W_{n}\left(\mathbb{F}_{p^{f}}\right)$ and the category $H D F_{p-2, f}\left(X_{n+1} / W_{n+1}\right)$.

Proof. Recall that we have chosen a primitive element $\xi_{1} \in \mathbb{F}_{p^{f}}$ in the proof of Lemma 3.8. Let $\xi$ be the Teichmüller lift of $\xi_{1}$ in $W\left(\mathbb{F}_{p^{f}}\right)$ and let $\xi_{n}$ be the mod $p^{n}$ reduction of $\xi$. Thus $\xi_{n}$ is a generator of $W_{n}\left(\mathbb{F}_{p^{f}}\right)$ as $W_{n}\left(\mathbb{F}_{p}\right)$-algebra. The Frobenius automorphism of $W_{n}=W_{n}(k)$ acts on $\xi_{n}$ by the power $p$ map. We prove by induction on $n$. The $n=1$ case is Proposition 3.7, where we have constructed 
the functor $\mathcal{E}$ and its quasi-inverse $\mathcal{D}$. Rewrite them into $\mathcal{E}_{1}$ and respectively $\mathcal{D}_{1}$. As the induction hypothesis, we assume that we have constructed a sequence of functors $\left\{\mathcal{E}_{i}\right\}_{1<i \leq n-1}$ and $\left\{\mathcal{D}_{i}\right\}_{1 \leq i \leq n-1}$ such that (i) $\mathcal{E}_{i}$ and $\mathcal{D}_{i}$ are quasi-inverse to each other and therefore give a equivalence of categories between the category of one-periodic Higgs-de Rham flows over $X_{i}$ with endomorphism structure $W_{i}\left(\mathbb{F}_{p^{f}}\right)$ and the category $H D F_{p-2, f}\left(X_{i}\right)$; (ii) $\mathcal{E}_{i}\left(\operatorname{resp} . \mathcal{D}_{i}\right)$ lifts $\mathcal{E}_{i-1}\left(\operatorname{resp} . \mathcal{D}_{i-1}\right)$. Now we proceed to show the case for $n$. Let us start with an object of $H D F_{p-2, f}\left(X_{n}\right)$ :

$$
\left(E, \theta, F i l_{0}, \cdots, F i l_{f-1}, \phi, \bar{E}, \bar{\theta}, \bar{F} i l_{0}, \cdots, \bar{F} i l_{f-1}, \bar{\phi}\right) .
$$

Following the characteristic $p$ case, we put

$$
(G, \eta):=\bigoplus_{i=0}^{f-1}\left(E_{i}, \theta_{i}\right)
$$

where $\left(E_{0}, \theta_{0}\right)=(E, \theta)$ and $\left(E_{i}, \theta_{i}\right)=C_{n}^{-1}\left(E_{i-1}, \theta_{i-1}\right), i \geq 1$ are the remaining Higgs terms defined inductively in the flow. The Hodge filtration $F i l$ on $C_{n}^{-1}(G, \eta)$ as well as the isomorphism $\tilde{\phi}$ of graded Higgs modules over $X_{n}$ are defined exactly in the same way as the characteristic $p$ case (see the paragraph before Lemma 3.8). Clearly, $(G, \theta)$ (resp. Fil and $\tilde{\phi}) \operatorname{lifts}(\bar{G}, \bar{\theta})$ (resp. Fil and $\overline{\tilde{\phi}}$ ) and therefore $(G, \eta, F i l, \tilde{\phi}, \bar{G}, \bar{\theta}, \overline{F i l}, \overline{\tilde{\phi}})$ is a one-periodic flow over $X_{n}$. Replacing $\xi_{1}$ in the proof of Lemma 3.8 with $\xi_{n}$, one equips this one-periodic flow with an endomorphism structure $W_{n}\left(\mathbb{F}_{p^{f}}\right)$. This gives us a functor $\mathcal{E}_{n}$. Conversely, to a one-periodic Higgs-de Rham flow $(G, \eta, F i l, \phi, \iota, \bar{G}, \bar{\eta}, \bar{F} i l, \bar{\phi})$ over $X_{n}$ with the endomorphism structure $W_{n}\left(\mathbb{F}_{p^{f}}\right)$, we associate an $f$-periodic Higgs-de Rham flow over $X_{n}$ as follows: first, the induction hypothesis gives us an $f$-periodic flow over $X_{n-1}$ (by abuse of notation we have omitted the part over $X_{n-1}$ in the following expression):

$$
\left(\bar{E}, \bar{\theta}, \widetilde{F i l_{0}}, \cdots, \widetilde{F i l_{f-1}}, \overline{\tilde{\phi}}\right) .
$$

Second, let $(G, \eta)=\oplus_{i=0}^{f-1}\left(G_{i}, \eta_{i}\right)$ be the eigen-decomposition under the endomorphism $\iota\left(\xi_{n}\right)$. Then $C_{n}^{-1}\left(G_{i}, \eta_{i}\right)$ is naturally defined and one has the eigendecomposition under $C_{n}^{-1}\left(\xi_{n}\right)$ :

$$
\left(C_{n}^{-1}(G, \eta), F i l\right)=\bigoplus_{i=0}^{f-1}\left(C_{n}^{-1}\left(G_{i}, \eta_{i}\right), F i l_{i}\right) .
$$

So we put $(E, \theta)=\left(G_{0}, \eta_{0}\right)$, and following strictly the constructions in the characteristic $p$ case (see Lemma [3.9), one obtains the filtrations $\widetilde{F i l}_{i}, 0 \leq i \leq f-1$ and the isomorphism $\tilde{\phi}$ of graded Higgs modules over $X_{n}$, so that the extended tuple

$$
\left(E, \theta, \widetilde{F i l_{0}}, \cdots, \widetilde{F i l_{f-1}}, \tilde{\phi}, \bar{E}, \bar{\theta}, \widetilde{F i l_{0}}, \cdots, \widetilde{F i l_{f-1}}, \overline{\tilde{\phi}}\right)
$$

is an object in $H D F_{p-2, f}\left(X_{n}\right)$. This give us the functor $\mathcal{D}_{n}$ in the reverse direction. Given the proof of Proposition [3.7, the proof for that $\mathcal{E}_{n}$ and $\mathcal{D}_{n}$ give an equivalence of categories becomes completely formal and is therefore omitted. Finally, the lifting properties of $\mathcal{E}_{n}$ and $\mathcal{D}_{n}$ are direct consequences of our choice of $\xi_{n}$ at the beginning. 
The remaining paragraphs are devoted to the proof of Proposition 5.4. In the following, we choose and then fix an open affine covering $\mathcal{U}=\left\{U_{i}\right\}_{i \in I}$ of $X_{n+1} / W_{n+1}$ whose element is flat over $W_{n+1}$, and for each $i$, an absolute Frobenius lifting $F_{i}$ over $U_{i}$. First a lemma:

Lemma 5.6. Let $(H, \nabla, F i l, \Phi)$ be a strict $p^{n}$-torsion Fontaine module. Let $(\bar{H}, \bar{\nabla}, \overline{F i l})$ be the $\bmod p^{n-1}$ reduction of $(H, \nabla, F i l)$ and $(E, \theta)$ the associated graded Higgs bundle. Then the relative Frobenius $\Phi$ naturally induces an isomorphism of flat bundles over $X_{n}$ :

$$
\tilde{\Phi}: C_{n}^{-1}(E, \theta) \cong(H, \nabla) .
$$

Proof. The lemma follows from the strong $p$-divisibility and horizontality of $\Phi$ (see $\S 2$ ) and the very construction of $C_{n}^{-1}$. For $n=1$, this is Proposition 1.4 [13. For simplicity, let us ignore the issue of the obvious base change caused by the Frobenius automorphism of the base ring $W_{n}$ in the argument. By the first approach of the functor $\mathcal{T}_{n}$, it follows that

$$
\mathcal{T}_{n}(E, \theta, \bar{H}, \bar{\nabla}, \bar{F} i l)=G_{n}(H, \nabla, F i l) .
$$

Write it to be $(\tilde{H}, \tilde{\nabla})$. Let $\mathcal{U}_{n}=\left\{U_{i, n}\right\}_{i \in I}$ be the induced open affine covering of $X_{n}$, and let $F_{i, n}$ be the induced absolute Frobenius lifting over $U_{i, n}$. Then the evaluation $\Phi_{i}:=\Phi_{\left(U_{i, n}, F_{i, n}\right)}$ of $\Phi$ is an isomorphism of local flat bundles:

$$
\Phi_{i}:\left.\left(\left.F_{i, n}^{*} \tilde{H}\right|_{U_{i, n}}, F_{i, n}^{*}\left(\left.\tilde{\nabla}\right|_{U_{i, n}}\right)\right) \cong(H, \nabla)\right|_{U_{i, n}},
$$

where the connection $F_{i}^{*}\left(\left.\tilde{\nabla}\right|_{U_{i, n}}\right)$ is defined via the formula (4.15.1). The fact that different evaluations of $\Phi$ are related via the Taylor formula means

$$
\Phi_{i}=\Phi_{j} \circ \varepsilon_{i j}
$$

where the $\varepsilon_{i j}$ is given by the formula (4.16.1) (replacing the indices 21 in that formula with $i j$ ). Thus, the very construction of $C_{n}^{-1}$ means exactly that the local isomorphisms $\Phi_{i}$ s glue together into a global one $\tilde{\Phi}$ from $C_{n}^{-1}(E, \theta)$ to $(H, \nabla)$.

Proof of Proposition 5.4. We divide the whole proof into three steps, following the one in the characteristic $p$ case. The proof is by induction on $n$, where the $n=1$ case is Proposition 3.3. Rewrite the functors in the proof of Proposition 3.3 by $\mathcal{G R}_{1}:=\mathcal{G} \mathcal{R}$ and $\mathcal{I} \mathcal{C}_{1}:=\mathcal{I C}$. As the induction hypothesis, we assume then the existence of functors $\mathcal{G} \mathcal{R}_{i}: M F_{[0, p-2], 1}^{\nabla}\left(X_{i+1} / W_{i+1}\right) \rightarrow H D F_{p-2,1}\left(X_{i+1} / W_{i+1}\right)$ and $\mathcal{I C}_{i}$ in the opposite direction for $1 \leq i \leq n-1$ such that (i) $\mathcal{G R}_{i}$ and $\mathcal{I C}_{i}$ are quasi-inverse to each other, and $(2) \mathcal{G} \mathcal{R}_{i}$ (resp. $\left.\mathcal{I} \mathcal{C}_{i}\right)$ lifts $\mathcal{G} \mathcal{R}_{i-1}$ (resp. $\mathcal{I C}_{i-1}$ ). In the following, we construct a lifting $\mathcal{G R}_{n}$ (resp. $\left.\mathcal{I C}_{n}\right)$ of $\mathcal{G R}_{n-1}\left(\right.$ resp. $\left.\mathcal{I C}_{n-1}\right)$ such that $\mathcal{G} \mathcal{R}_{n}$ and $\mathcal{I} \mathcal{C}_{n}$ are quasi-inverse to each other.

From Fontaine module to one-periodic Higgs-de Rham flow: Let $(H, \nabla, F i l, \Phi)$ be a strict $p^{n}$-torsion Fontaine module over $X_{n+1} / W_{n+1}$. Its reduction $\bmod p^{n-1}$ $(\bar{H}, \bar{\nabla}, \bar{F} i l, \bar{\Phi})$ is a strict $p^{n-1}$-torsion Fontaine module. Let

$$
\left(\bar{E}, \bar{\theta}, \overline{F i} l_{e x p}, \bar{\phi}, \overline{\bar{E}}, \overline{\bar{\theta}}, \overline{\bar{F}} i l_{e x p}, \overline{\bar{\phi}}\right)
$$


be the corresponding one-periodic flow over $X_{n-1}$ to $(\bar{H}, \bar{\nabla}, \bar{F} i l, \bar{\Phi})$ via the functor $\mathcal{G R}_{n-1}$. Set $(E, \theta)=G r_{F i l}(H, \nabla)$. By Lemma [5.6, we define $F i l_{\text {exp }}$ on $C_{n}^{-1}(E, \theta)$ to be the pull-back of Fil on $H$ via the isomorphism $\tilde{\Phi}$. Thus, we obtain also an isomorphism of graded Higgs modules over $X_{n}$ :

$$
\phi:=\operatorname{Gr}(\tilde{\Phi}): G r_{\text {Fil exp }}\left(C_{n}^{-1}(E, \theta)\right) \cong G r_{F i l}(H, \nabla)=(E, \theta) .
$$

The lifting property of the inverse Cartier transform in Theorem 4.1 implies that the so-obtained tuple $\left(E, \theta, F i l_{\text {exp }}, \phi\right)$ lifts $\left(\bar{E}, \bar{\theta}, \bar{F} i l_{e x p}, \bar{\phi}\right)$, so that

$$
\mathcal{G R}_{n}(H, \nabla, F i l, \Phi):=\left(E, \theta, F i l_{\text {exp }}, \phi, \bar{E}, \bar{\theta}, \bar{F} i l_{\text {exp }}, \bar{\phi}\right)
$$

is a one-periodic Higgs-de Rham flow over $X_{n}$. Clearly, the functor $\mathcal{G R}_{n}$ lifts $\mathcal{G R}_{n-1}$.

From one-periodic Higgs-de Rham flow to Fontaine module: From a given object $(E, \theta, F i l, \phi, \bar{E}, \bar{\theta}, \bar{F} i l, \bar{\phi}) \in H D F_{p-2,1}\left(X_{n}\right)$, one derives immediately the de Rham module

$$
(H, \nabla, F i l):=\left(C_{n}^{-1}(E, \theta), \text { Fil }\right) .
$$

In order to complete it into a Fontaine module, it remains to put a relative Frobenius $\Phi$ on it. Let $(\bar{H}, \bar{\nabla})$ be the mod $p^{n-1}$ reduction of $(H, \nabla)$ which is equal to $C_{n-1}^{-1}(\bar{E}, \bar{\theta})$. Set $\left(E_{1}, \theta_{1}\right)=G_{F i l}(H, \nabla)$. Then we have two objects in the category $\mathcal{H}\left(X_{n}\right):(E, \theta, \bar{H}, \bar{\nabla}, \bar{F} i l, \bar{\phi})$ and $\left(E_{1}, \theta_{1}, \bar{H}, \bar{\nabla}, \bar{F} i l, I d\right)$. Then $\phi$ : $\left(E_{1}, \theta_{1}\right) \cong(E, \theta)$ and the identity map on $(\bar{H}, \bar{\nabla}, \bar{F} i l)$ give rise to an isomorphism

$$
\phi:\left(E_{1}, \theta_{1}, \bar{H}, \bar{\nabla}, \bar{F} i l, I d\right) \cong(E, \theta, \bar{H}, \bar{\nabla}, \bar{F} i l, \bar{\phi}) .
$$

and therefore an isomorphism $\tilde{\phi}:=\mathcal{T}_{n}(\phi)$ of twisted flat modules after Proposition 4.11

$$
(\tilde{H}, \tilde{\nabla}):=\mathcal{T}_{n}\left(E_{1}, \theta_{1}, \bar{H}, \bar{\nabla}, \bar{F} i l, I d\right) \cong \mathcal{T}_{n}(E, \theta, \bar{H}, \bar{\nabla}, \bar{F} i l, \bar{\phi}):=\left(\tilde{H}_{-1}, \tilde{\nabla}_{-1}\right) .
$$

Note the isomorphism $\mathcal{T}_{1}(\phi)$ for $n=1$ is nothing but the original isomorphism $\phi$ between graded Higgs bundles. Then for an open affine covering of $X_{n+1}$ and the set of Frobenius liftings as given in Lemma 5.6, following the method in the characteristic $p$ case, we define simply an $\mathcal{O}_{U_{i}}$-isomorphism by

$$
\Phi_{i}=F_{i, n}^{*}(\tilde{\phi}): F_{i, n}^{*} \tilde{H} \cong F_{i, n}^{*}\left(\tilde{H}_{-1}\right),
$$

where the latter module is just $\left.H\right|_{U_{i, n}}=\left.C_{n}^{-1}(E, \theta)\right|_{U_{i, n}}$. At this point, the triple $\left(\left.H\right|_{U_{i, n}},\left.F i l\right|_{U_{i, n}}, \Phi_{i}\right)$ is a local $p$-torsion Fontaine module without connection (i.e. it is an object in the local category $M F\left(R_{i, n}\right)$ with $R_{i, n}=\Gamma\left(U_{i, n}, \mathcal{O}_{U_{i, n}}\right)$ as given in Page 31 [4). By Theorem 2.1 [4 ii), one knows that $H$ is a locally free $\mathcal{O}_{X_{n}}$-module. Moreover, by the construction of the functor $\mathcal{F}_{n}, \Phi_{i}$ is indeed an isomorphism of flat modules:

$$
\Phi_{i}:\left(\left.F_{i, n}^{*} \tilde{H}\right|_{U_{i, n}},\left.\left.F_{i, n}^{*} \tilde{\nabla}\right|_{U_{i, n}} \cong(H, \nabla)\right|_{U_{i, n}} .\right.
$$

This gives the horizontal property as required by an evaluation of the relative Frobenius (see Variant $1 \S 2$ ). It remains to explain $\Phi_{i}$ s are related via the Taylor 
formula (5.6.1). Let $e$ be a local section of $\tilde{H}$ over $U_{i, n}$. Then one computes that over $U_{i j, n}:=U_{i, n} \cap U_{j, n}$,

$$
\begin{aligned}
G_{i j} \circ \Phi_{i}(e \otimes 1) & =\sum_{J} \frac{\tilde{\nabla}_{-1}(\partial)^{J}(\tilde{\phi}(e))}{J !} \otimes z^{J} \\
& =\sum_{J} \frac{\tilde{\phi}\left(\tilde{\nabla}(\partial)^{J}(e)\right)}{J !} \otimes z^{J} \\
& =\Phi_{j}\left(\sum_{J} \frac{\tilde{\nabla}(\partial)^{J}(e)}{J !} \otimes z^{J}\right)=\Phi_{j} \circ \varepsilon_{i j}(e \otimes 1) .
\end{aligned}
$$

The second equality follows from the property that $\tilde{\phi}$ respects the twisted connections, and the last equality follows from the transition over $U_{i j, n}$ of a local section of $\tilde{H}$ over $U_{i, n}$ to a local section of $\tilde{H}$ over $U_{j, n}$. So we obtain a relative Frobenius $\Phi$ from local $\Phi_{i}$ s and thus a strict $p^{n}$-torsion Fontaine module over $X_{n}$ :

$$
\mathcal{I C}_{n}(E, \theta, F i l, \phi, \bar{E}, \bar{\theta}, \bar{F} i l, \bar{\phi}):=(H, \nabla, F i l, \Phi) .
$$

That the functor $\mathcal{I C}_{n}$ lifts $\mathcal{I C}_{n}$ follows from the lifting property of the inverse Cartier transform and the construction of the relative Frobenius.

Equivalence of categories: This is done by induction on $n$ and the constructions of the natural transformations in the proof of Proposition 3.3 .

At this point, we have completed our theory on the Higgs correspondence in positive and mixed characteristic (§3-§5). In [12] (see also [11, Remark 5.5]), N. Katz established the following result.

Theorem 5.7 (Proposition $4.1 .1[12]$ ). Let $X_{n} / W_{n}$ be an irreducible smooth affine scheme over $W_{n}$, equipped with an absolute Frobenius lifting $F_{X_{n}}$. Then there is an equivalence of categories between the category of $W_{n}\left(\mathbb{F}_{p^{f}}\right)$-representations of $\pi_{1}\left(X_{n}\right)$ and the category of pairs $(E, \phi)$ consisting of a locally free sheaf of finite rank $E$ over $X_{n}$ together with an isomorphism $\phi: F_{X_{n}}^{* f}(E) \rightarrow E$.

The Katz's correspondence has been further developed in the work [3] of EmertonKisin, where the role of unit $F$-crystals is emphasized. A pair $(E, \phi)$ in Theorem 5.7 is called a Frobenius-periodic vector bundle over $X_{n}$. This is the prototype of the notion of perodic Higgs-de Rham flow. Indeed, to a Frobeniusperodic vector bundle $(E, \phi)$, one associates the perodic Higgs-de Rham flow $\left(E, 0, F i l_{t r}, \cdots, F i l_{t r}, \phi\right)$ over $X_{n}$ of level zero, and this association is an equivalence of categories. Take an arbitrary $W$-lifting $X=\operatorname{Spec} R$ of $X_{n}$ and $\Gamma$ (resp. $\Gamma^{u r}$ ) the Galois group of the maximal extension of $R$ étale over $R[1 / p]$ (resp. over $R$ ) (Ch. II [4]). Then, one may show that the corresponding $W_{n}\left(\mathbb{F}_{p^{f}}\right)$ representation of $\Gamma$ to $\left(E, 0, F i l_{t r}, \cdots, F i l_{t r}, \phi\right)$ over $X_{n}$ after Theorem 5.3 and Theorem 2.6 [4] factors through the natural quotient $\Gamma \rightarrow \Gamma^{u r}=\pi_{1}\left(X_{n}\right)$, and the resulting representation of $\pi_{1}\left(X_{n}\right)$ coincides with the representation corresponding to $(E, \phi)$ after Theorem 5.7. Moreover, our theory extends to the case where only the existence of $W_{n+1}$-lifting of $X_{n}$ is assumed. Namely, one may show that, 
given a $W_{n+1}$-lifting $X_{n+1}$ of $X_{n}$, there is an equivalence of categories between the category of $f$-periodic Higgs-de Rham flow over $X_{n}$ of level zero and the category of $W_{n}\left(\mathbb{F}_{p^{f}}\right)$-representations of $\pi_{1}\left(X_{n}\right)$.

To demonstrate the usage of the theory in a higher Hodge-Tate weight situation, we provide an immediate construction of a $p$-divisible group over a geometric base over $W$ whose Kodaira-Spencer map is an isomorphism.

Example 5.8. Let $A_{1}$ be any ordinary abelian variety defined over $k$ of dimension $g$. By Serre-Tate theory, it has the canonical lifting $A$ over $W(k)$ with the Frobenius lifting $F: A \rightarrow A$. Consider the following Higgs bundle $(E, \theta)$ over $A / W$ :

$$
E^{1,0} \oplus E^{0,1}=\Omega_{A} \oplus \mathcal{O}_{A}, \quad \theta^{1,0}=I d: \Omega_{A} \rightarrow \mathcal{O}_{A} \otimes \Omega_{A} .
$$

In the following we show that this Higgs bundle is one-periodic. We set $\left(E_{n}, \theta_{n}\right):=$ $(E, \theta) \otimes \mathbb{Z} / p^{n} \mathbb{Z}$, and $F_{n}=F \otimes \mathbb{Z} / p^{n} \mathbb{Z}$. First we construct a one-periodic Higgs-de Rham flow on $A_{1}$ : since $A_{2}$ has the global Frobenius lifting $F_{2}$, it follows that

$$
C_{1}^{-1}\left(E_{1}, \theta_{1}\right):=\left(H_{1}, \nabla_{1}\right),
$$

with

$$
H_{1}:=F_{1}^{*} E_{1} \quad \text { and } \quad \nabla_{1}=\nabla_{c a n}+\frac{d F_{2}}{p}\left(F_{1}^{*} \theta_{1}\right)
$$

A Hodge filtration of level one on $\left(H_{1}, \nabla_{1}\right)$ is defined by $F i l_{1}^{1}=F_{1}^{*} \Omega_{A_{1}}=\Omega_{A_{1}}$. Set

$$
\left(E_{1}^{\prime}, \theta_{1}^{\prime}\right):=G r_{F i l_{1}}\left(H_{1}, \nabla_{1}\right)
$$

Then

$$
E_{1}^{\prime}=\Omega_{A_{1}} \oplus \mathcal{O}_{A_{1}}, \quad \theta_{1}^{\prime 1,0}=\frac{d F_{2}}{p}\left(F_{1}^{*} \theta_{1}\right)
$$

Because of the ordinariness of $A_{1}$, the Hasse-Witt map

$$
\frac{d F_{2}}{p}: H^{0}\left(A_{1}, \Omega_{A_{1}}\right) \rightarrow H^{0}\left(A_{1}, \Omega_{A_{1}}\right)
$$

is bijective. So $\theta_{1}^{\prime 1,0}$ has to be an isomorphism. Then we proceed to show $\left(E_{1}^{\prime}, \theta_{1}^{\prime}\right)$ is isomorphic to $\left(E_{1}, \theta_{1}\right)$. Indeed, there is one natural choice $\psi_{1}: E_{1}^{\prime} \rightarrow E_{1}$ of isomorphisms described as follows: its $(0,1)$-component mapping $\mathcal{O}_{A_{1}}$ to itself is the identity, and its $(1,0)$-component mapping $\Omega_{A_{1}}$ to itself is the unique isomorphism commuting with the Higgs fields. Therefore, we have obtained a one-periodic flow over $A_{1}$ as claimed:

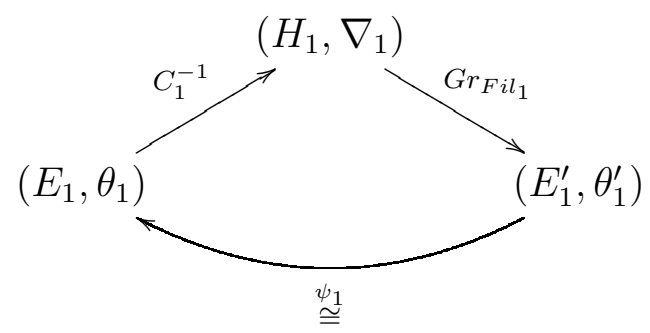


Next we proceed to the $W_{2}$-level. Using the fact that $A_{3}$ has the Frobenius lifting $F_{3}$, one computes that $\tilde{H}_{-1,2}=\Omega_{A_{2}} \oplus \mathcal{O}_{A_{2}}$, and $\tilde{\nabla}_{-1,2}=p \nabla_{c a n}+\theta_{2}$. Using Remark 4.17, it follows that

$$
C_{2}^{-1}\left(E_{2}, \theta_{2}\right)=\left(H_{2}, \nabla_{2}\right)
$$

with $H_{2}=F_{2}^{*} E_{2}=\Omega_{A_{2}} \oplus \mathcal{O}_{A_{2}}$ and $\nabla_{2}$ is the connection defined by the formula

$$
\nabla_{2}(f \otimes e)=d f \otimes e+f \cdot\left(\frac{d F_{3}}{p} \otimes 1\right)\left(1 \otimes \tilde{\nabla}_{-1,2}(e)\right)
$$

where $f$ (resp. $e$ ) is a local section of $\mathcal{O}_{A_{2}}$ (resp. $\tilde{H}_{-1,2}$ ) (see Claim 4.16). Now we take the filtration $F i l_{2}^{1}=\Omega_{A_{2}}$, which lifts $F i l_{1}^{1}$. Then the associated graded Higgs bundle is

$$
E_{2}^{\prime}=\Omega_{A_{2}} \oplus \mathcal{O}_{A_{2}}, \theta_{2}^{\prime 1,0}=\frac{d F_{3}}{p}\left(F_{2}^{*} \theta_{2}\right)
$$

which lifts $\left(E_{1}^{\prime}, \theta_{1}^{\prime}\right)$. Again there is an obvious isomorphism

$$
\psi_{2}:\left(E_{2}^{\prime}, \theta_{2}^{\prime}\right) \rightarrow\left(E_{2}, \theta_{2}\right)
$$

which lifts $\psi_{1}$ and whose $(0,1)$-component is the identity map. So we obtain a one-periodic flow over $A_{2}$ :

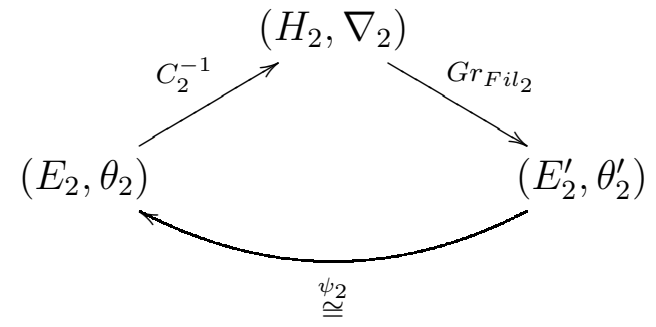

Then one continues and constructs inductively a one-periodic flow over $A_{n}, n \geq 1$ :

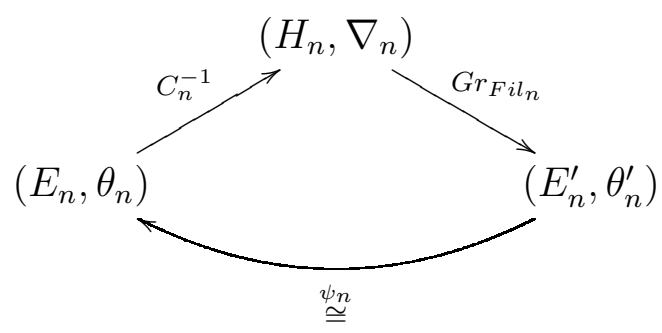

Passing to the limit, one obtains therefore a one-periodic Higgs-de Rham flow over $A / W$, and hence a rank $g+1$ crystalline $\mathbb{Z}_{p}$-representation of Hodge-Tate weight one of the generic fiber $A^{0}$ of $A / W$ which by [4, Theorem 7.1] corresponds to a $p$-divisible group over $A / W$.

\section{Strongly semistable Higgs modules}

Let $X / k$ be a smooth projective variety over $k$, equipped with an ample divisor $Z$. The semistability in this section means the $\mu_{Z}$-slope semistability. Recall that a vector bundle over $X$ is said to be strongly semistable if the bundle as well as its pullback under any power of Frobenius are semistable. The relation between strongly semistable bundles with trivial Chern classes and representations of the algebraic fundamental group was firstly revealed by Lange-Stuhler in the curve 
case (see [21, §1]). It asserts that a semistable vector bundle $E$ of degree zero over a curve can be trivialized after a finite morphism if and only if it is strongly semistable ([21, Satz 1.9]). Note that $E$ being strongly semistable of degree zero is equivalent to the condition that there is a pair $(e, f)$ of integers with nonnegative $e$ and positive $f$ such that

$$
F_{X}^{* e+f}(E) \cong F_{X}^{* e}(E)
$$

where $F_{X}: X \rightarrow X$ denotes the absolute Frobenius morphism as usual. It corresponds to a representation of $\pi_{1}(X)$ into $\mathrm{GL}(k)$ if and only if $e$ in the above isomorphism can be taken to be zero ([21, Proposition 1.2, Satz 1.4]). The result of Lange-Stuhler has been generalized to a singular curve by Deninger-Werner (see [2, Theorem 18]) and this generalization played a key role in their partial $p$-adic analogue of the Narasimhan-Seshadri theory. Besides the intimate relation with the representation of $\pi_{1}$, the notion of strongly semistability is also useful in other situation, for example, in Langer's proof of boundedness of semistable sheaves and Bogomolov's inequality in positive characteristic [17]. Therefore, it is a natural question to generalize this notion to Higgs modules. Interestingly enough, it turns out that our generalization (especially Theorem 6.5 below) has played a key role in the very recent result, due to A. Langer [20], on the Bogomolov-Gieseker inequality for semistable Higgs bundles and Miyaoka-Yau inequality for surfaces in positive characteristic.

The key of the generalization is to replace the Frobenius pullback with the inverse Cartier transform of Ogus-Vologodsky [25], as seen in the following

Definition 6.1. Let $X$ be a smooth projective variety over $k$ together with a fixed $W_{2}$-lifting of $X$. A Higgs module $(E, \theta)$ is called strongly semistable if it appears in the initial term of a semistable Higgs-de Rham flow, that is, all Higgs terms $\left(E_{i}, \theta_{i}\right) \mathrm{s}$ in the flow are semistable and defined over a common finite subfield of $k 1$

A torsion Higgs module is by definition automatically semistable, which is however uninteresting to study in the current setting. Therefore, a semistable Higgs module is taciturnly assumed to be torsion free in this paper. Clearly, a strongly semistable vector bundle $E$ is strongly Higgs semistable: one takes simply the following Higgs-de Rham flow

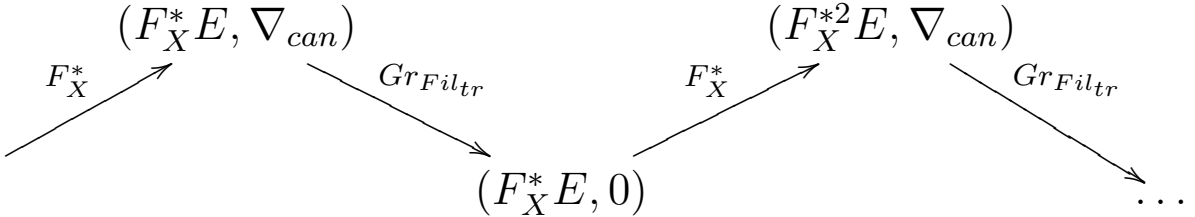

where $\nabla_{\text {can }}$ is the canonical connection in the theorem of Cartier descent and $F i l_{t r}$ stands for the trivial filtration as before.

\footnotetext{
${ }^{1}$ This definition corrects the error in [14, Definition 2.1].
} 
The first result on the Higgs semistability of the graded Higgs module associated to a strict $p$-torsion Fontaine module is due to Ogus-Vologodsky [25, Proposition 4.19].

Proposition 6.2 (Ogus-Vologodsky). Let $X / k$ be a smooth projective curve of genus $g$. Let $(H, \nabla, F i l, \Phi)$ be a strict p-torsion Fontaine module (with respect to some $W_{2}$-lifting of $\left.X\right)$. Assume that

$$
n(\operatorname{rank} H-1) \max \{2 g-2,1\}<p-1 .
$$

Then $\operatorname{Gr}_{F i l}(H, \nabla)$ is a semistable Higgs bundle.

Their result can be generalized as follows (see [26, Proposition 0.2] for a generalization in the geometric case as given in Example 2.2] and see also [28, Proposition $3.7])$ :

Proposition 6.3. Let $X / k$ be a smooth projective variety. Let $(H, \nabla, F i l, \Phi)$ be a strict $p$-torsion Fontaine module (with respect to some $W_{2}$-lifting of $X$ ). Then the graded Higgs bundle $(E, \theta):=G_{F i l}(H, \nabla)$ is Higgs semistable. Moreover, any Higgs subsheaf $(G, \theta) \subset(E, \theta)$ of slope zero is strongly semistable.

Proof. Let us first recall that we have proven that there is a natural isomorphism

$$
\tilde{\Phi}: C_{1}^{-1}(E, \theta) \cong(H, \nabla) .
$$

See [13, Proposition 1.4]. Therefore, one obtains a natural isomorphism $G r_{F i l}$ 。 $C_{1}^{-1}(E, \theta) \cong(E, \theta)$. It implies that $p \mu(E)=\mu(E)$, and thus $\mu(E)=0$. For the first statement, we prove by contradiction. Let $(F, \theta)$ be the maximal destabilizing Higgs subsheaf of $(E, \theta)$ with positive slope $\mu(F)$. Then $G r_{F i l} \circ C_{1}^{-1}(F, \theta)$ is naturally a Higgs subsheaf of slope $p \mu(F)>\mu(F)$. A contradiction. For the second statement, let us first notice that a Higgs subsheaf $(G, \theta)$ of slope zero is automatically semistable, as firstly observed by C. Seshadri. Now that $G r_{F i l}$ 。 $C_{1}^{-1}(G, \theta)$ is naturally a Higgs subsheaf of $(E, \theta)$ which is again of slope zero and hence semistable, $(G, \theta)$ is strongly semistable.

It is surprising to have the following

Proposition 6.4. Notation as above. Any rank two nilpotent semistable Higgs module is strongly semistable.

Proof. Let $(E, \theta)$ be a rank two nilpotent semistable Higgs module over $X$. For the reason of rank, $\theta$ is nilpotent of exponent $\leq 1$. Denote $(H, \nabla)$ for $C_{1}^{-1}(E, \theta)$, and $H N$ the Harder-Narasimhan filtration on $H$. We need to show that the graded Higgs module $G r_{H N}(H, \nabla)$ is again semistable. If $H$ is semistable, there is nothing to prove: in this case, the $H N$ is trivial and hence the induced Higgs field is zero, and $G r_{H N}(H, \nabla)=(H, 0)$ is Higgs semistable. Denote otherwise $L_{1} \subset H$ for the invertible subsheaf of maximal slope and $L_{2}=H / L_{1}$ the quotient sheaf. Then $L_{1}$ cannot be $\nabla$-invariant. Indeed, $(H, \nabla)$ is $\nabla$-semistable as a general property: say $M \subset H$ any $\nabla$-invariant subsheaf. Then $\left(F,\left.\theta\right|_{F}\right):=C_{1}\left(M,\left.\nabla\right|_{M}\right) \subset C_{1}(H, \nabla)=$ $(E, \theta)$ is a Higgs subsheaf of slope $\mu(F)=\mu(M) / p$, where $C_{1}$ is the Cartier 
transform of Ogus-Vologodsky (see also [13]). As $(E, \theta)$ is Higgs semistable, it follows that

So the natural map

$$
\mu(M)=p \mu(F) \leq p \mu(E)=\mu(H) .
$$

$$
\theta^{\prime}=G r_{H N} \nabla: L_{1} \rightarrow L_{2} \otimes \Omega_{X_{1}}
$$

is nonzero. A nontrivial proper Higgs subsheaf $L \subset G r_{H N}(H, \nabla)$ is simply an invertible subsheaf with $\theta^{\prime}(L)=0$. So $L \subset L_{2}$ and

$$
\mu(L) \leq \mu\left(L_{2}\right)<\mu(H)=\mu\left(G r_{H N} H\right) .
$$

In this case, $\operatorname{Gr}_{H N}(H, \nabla)$ is actually Higgs stable.

Motivated by Proposition 6.4, we proposed a conjecture on strongly semistability of a nilpotent semistable Higgs module of higher rank in the first version of the paper (see [14, Conjecture 2.8]). The conjecture was proven by A. Langer in [19] in the case of small rank, which is quite crucial to his algebraic proof of Bogomolov-Gieseker inequality and Miyaoka-Yau inequality. In Appendix A, we shall provide an independent proof of the conjecture in the case of small rank.

Theorem 6.5 (Theorem A.1, [19, Theorem 5.1]). Notation as above. Any nilpotent semistable Higgs module of rank $\leq p$ over $X$ is strongly semistable.

After establishing the notion of strongly semistability and exhibiting ample examples, we shall concentrate on those strongly semistable Higgs modules with trivial Chern classes, as guided by the theorem of Lange-Stuhler in the beginning. It turns out they are quite close to be periodic.

Theorem 6.6. Let $X / k$ be a smooth projective variety together with a fixed $W_{2}$ lifting of $X$. A preperiodic Higgs module is strongly semistable with trivial Chern classes. Conversely, a strongly semistable Higgs module with trivial Chern classes is preperiodic.

Proof. For a Higgs module $(E, \theta) \in H I G_{p-1}(X)$, let $(H, \nabla)=C_{1}^{-1}(E, \theta)$ be the corresponding flat module. It follows from the proof of [25, Theorem 4.17] that

$$
c_{l}(H)=p^{l} c_{l}(E), l \geq 0 .
$$

Since for any Griffiths transverse filtration $F i l$ on $(H, \nabla)$ the associated graded Higgs module $\left(E^{\prime}, \theta^{\prime}\right)=G r_{F i l} \circ C_{1}^{-1}(E, \theta)$ has the same Chern classes as $H$, it follows that

$$
c_{l}\left(E^{\prime}\right)=p^{l} c_{l}(E), l \geq 0 .
$$

Therefore, in a Higgs-de Rham flow, one has for $i \geq 0$

$$
c_{l}\left(E_{i+1}\right)=p^{l} c_{l}\left(E_{i}\right), l \geq 0 .
$$

This forces the Chern classes of a preperiodic Higgs module to be trivial. Also, a slope $\lambda$ Higgs subsheaf in $\left(E_{i}, \theta_{i}\right)$ gives rise to a slope $p \lambda$ Higgs subsheaf in $\left(E_{i+1}, \theta_{i+1}\right)$. This implies that, in a preperiodic Higgs-de Rham flow, each Higgs term $\left(E_{i}, \theta_{i}\right)$ contains no Higgs subsheaf of positive degree. So each $\left(E_{i}, \theta_{i}\right)$ is semistable. This shows the first statement. Conversely, let $(E, \theta)$ be a strongly semistable Higgs module with trivial Chern classes, and let $\left(E_{i}, \theta_{i}\right)_{i \geq 0}$ be the Higgs terms appearing in a semistable Higgs-de Rham flow with the initial term $(E, \theta)$. 
As discussed above, each $\left(E_{i}, \theta_{i}\right)$ has trivial Chern classes (and the same rank as $E)$. By [20, Lemma 5] and [19, Theorem 4.4], the moduli space of semistable Higgs modules with trivial Chern classes over $X / k$ is bounded and defined over $k$. Let $k^{\prime} \subset k$ be the common finite subfield for the infinite sequence $\left\{\left(E_{i}, \theta_{i}\right)\right\}_{i \geq 0}$. Since any scheme of finite type over $k$ has only finitely many $k^{\prime}$-rational points, there must exist a pair of integers $(e, f) \in \mathbb{Z}_{\geq 0} \times \mathbb{N}$ such that there is an isomorphism (over $k$ ) of Higgs modules $\left(E_{e+f}, \theta_{e+f}\right) \cong\left(E_{e}, \theta_{e}\right)$. Certainly, one can make the above isomorphism into an isomorphism of graded Higgs modules by adjusting one of their gradings. Therefore, $(E, \theta)$ is preperiodic.

Using the notion of strongly semistable Higgs module as bridge, we can produce crystalline $k$-representations (up to isomorphism) from semistable nilpotent Higgs bundles (of small rank) with trivial Chern classes.

Theorem 6.7. Let $X / W$ be a smooth projective scheme. Then for any rank $r \leq$ $p-1$ semistable nilpotent Higgs bundle $(E, \theta)$ over $X_{k}$ with trivial Chern classes, one associates a unique $r$-dimensional crystalline $k$-representation of $\pi_{1}\left(X_{K}\right)$ up to isomorphism.

The proof relies on the next result which follows directly from Theorem 6.5 and Theorem 6.6.

Corollary 6.8. Notation as Theorem 6.7. Then any rank $r \leq p$ semistable nilpotent Higgs bundle over $X_{k}$ with trivial Chern classes is preperiodic.

It is a nontrivial problem to decide when a small rank semistable graded Higgs bundle with trivial Chern classes is periodic (and its period when it is indeed the case), albeit always preperiodic by the corollary. We shall discuss this problem as well as the basic properties of representations constructed in Theorem 6.7 on a later occasion.

More preparations are needed before we can prove Theorem 6.7. A (pre)peridoic Higgs bundle may lead more than one (pre)periodic Higgs-de Rham flows. However, in the setting of Corollary 6.8, there is a natural choice of filtrations in a preperiodic Higgs-de Rham flow.

Proposition 6.9 (Theorem A.4, Remark A.9, [19, Theorem 5.5]). Let $(H, \nabla)$ be $a \nabla$-semistable flat bundle over $X_{k}$. Then there exists a uniquely defined reduced gr-semistable filtration on $(H, \nabla)$ which is preserved by any automorphism of $(H, \nabla)$.

The reader is referred to the appendix for the definition of a gr-semistable (resp. reduced) filtration. We shall call the filtration in the above result the Simpson filtration and denote it by $F i l_{S}$. Note that the Simpson filtration in the rank two case is nothing but the Harder-Narasimhan filtration on $H$, and it may well differ from the Harder-Narasimhan filtration for higher ranks. A periodic Higgs-de Rham flow whose each de Rham term is equipped with the Simpson filtration is unique up to lengthening, as proven below. The unicity is shared more generally by a periodic Higgs-de Rham flow with the following 
Assumption 6.10. Let $\left(E, \theta, F i l_{0}, \cdots, F i l_{f-1}, \varphi\right)$ be a periodic Higgs-de Rham flow over $X_{k}$. Assume that, for each $0 \leq i \leq f-1$, the filtration $F i l_{i}$ on $H_{i}$ is preserved by any automorphism of $\left(H_{i}, \nabla_{i}\right)$.

Recall in the definition of the lengthening, one has the following isomorphisms of graded Higgs modules induced by $\varphi:\left(E_{f}, \theta_{f}\right) \cong\left(E_{0}, \theta_{0}\right)$ :

$$
\left(G r C_{1}^{-1}\right)^{n f}(\varphi):\left(E_{(n+1) f}, \theta_{(n+1) f}\right) \cong\left(E_{n f}, \theta_{n f}\right), \quad n \in \mathbb{N} .
$$

Set, for $-1 \leq i<j$,

$\varphi_{j, i}=\left(G r C_{1}^{-1}\right)^{(i+1) f}(\varphi) \circ \cdots \circ\left(G r C_{1}^{-1}\right)^{j f}(\varphi):\left(E_{(j+1) f}, \theta_{(j+1) f}\right) \cong\left(E_{(i+1) f}, \theta_{(i+1) f}\right)$.

Note $\varphi_{j,-1}$ is just the isomorphism $\varphi_{j}$ in the $j$-th lengthening of the starting periodic flow.

Lemma 6.11. Let $\phi:\left(E_{f}, \theta_{f}\right) \cong\left(E_{0}, \theta_{0}\right)$ be another isomorphism of graded Higgs modules. Then there exists a pair $(i, j)$ with $0 \leq i<j$ such that $\phi_{j, i} \circ \varphi_{j, i}^{-1}=I d$.

Proof. If we denote $\tau_{s}=\phi_{s} \circ \varphi_{s}^{-1}$, then $\tau_{s}$ is an automorphism of $\left(E_{0}, \theta_{0}\right)$. Moreover, each element in the set $\left\{\tau_{s}\right\}_{s \in \mathbb{N}}$ is defined over the same finite field in $k$. As this is a finite set, there are $j>i \geq 0$ such that $\tau_{j}=\tau_{i}$. So the lemma follows.

Proposition 6.12. Assume Assumption 6.10. Let $(i, j)$ be a pair given by Lemma 6.11 for two given isomorphisms $\varphi, \phi:\left(E_{f}, \theta_{f}\right) \cong\left(E_{0}, \theta_{0}\right)$. Then there is an isomorphism in $H D F_{n,(j-i) f}\left(X_{2} / W_{2}\right)$ :

$$
\left(E, \theta, F i l_{0}, \cdots, F i l_{(j-i) f-1}, \varphi_{j-i-1}\right) \cong\left(E, \theta, F i l_{0}, \cdots, F i l_{(j-i) f-1}, \phi_{j-i-1}\right),
$$

where both sides of the isomorphism are obtained by $j-i-1$-th lengthening.

Proof. Put $\beta=\phi_{i} \circ \varphi_{i}^{-1}:\left(E_{0}, \theta_{0}\right) \cong\left(E_{0}, \theta_{0}\right)$. We shall check that it induces an isomorphism in $H D F_{n,(j-i) f}\left(X_{2} / W_{2}\right)$. By Assumption 6.10, $C_{1}^{-1}\left(G r C_{1}^{-1}\right)^{m}(\beta)$ for $m \geq 0$ always respects the filtrations. We need only to check that $\beta$ is compatible with $\phi_{j-i-1}$ as well as $\varphi_{j-i-1}$. So it suffices to show that the following diagram is commutative:

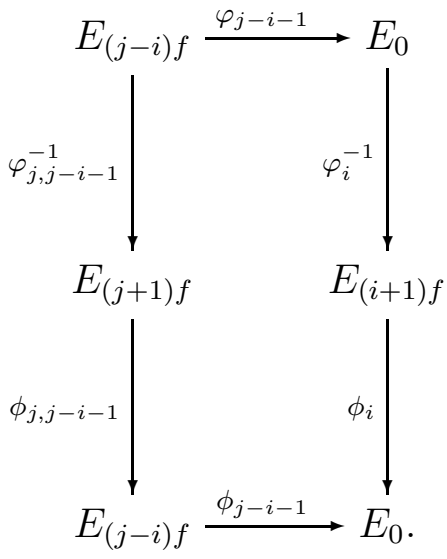


Clearly, it is equivalent to show the commutativity of the following diagram:

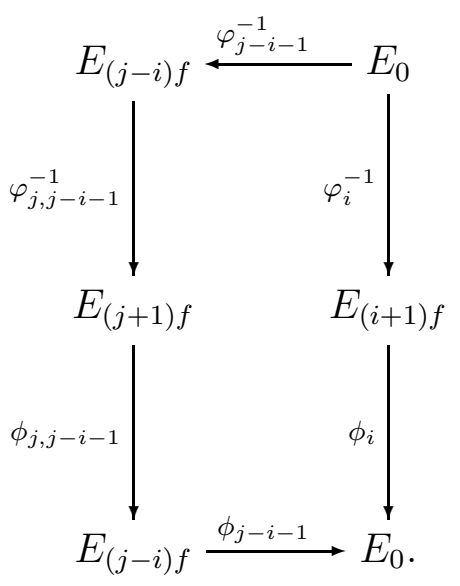

In the above diagram, the anti-clockwise direction is

$$
\phi_{j-i-1} \circ \phi_{j, j-i-1} \circ \varphi_{j, j-i-1}^{-1} \circ \varphi_{j-i-1}^{-1}=\phi_{j} \circ \varphi_{j}^{-1}=\phi_{i} \circ\left(\phi_{j, i} \circ \varphi_{j, i}^{-1}\right) \circ \varphi_{i} .
$$

By the requirement for $(i, j)$, we have $\phi_{j, i} \circ \varphi_{j, i}^{-1}=I d$, so the anti-clockwise direction is $\phi_{i} \circ \varphi_{i}$, which is exactly the clockwise direction. So $\beta$ is shown to be compatible with $\phi_{j-i-1}$ and $\varphi_{j-i-1}$.

Now we proceed to the proof of Theorem 6.7 .

Proof. It is to assemble the previous results. First, by Theorem 6.8, there exists a preperiodic flow with the initial term $(E, \theta)$. But there are several choices. In order to make a unique choice, we shall apply Proposition 6.9 at each step, that is, we use the Simpson filtration $F i l_{S}$ on each flat bundle and then we obtain the uniqueness on the filtrations in the flow. Now let $e \in \mathbb{N}_{0}$ be the minimal number such that $\left(G r_{F i l_{S}} \circ C_{1}^{-1}\right)^{e}(E, \theta)$ is periodic and let $f \in \mathbb{N}$ be its period. Thus from $(E, \theta)$ we have obtained an periodic Higgs-de Rham flow

$$
\left(\left(G_{F i l_{S}} \circ C_{1}^{-1}\right)^{e}(E, \theta), F i l_{0}=F i l_{S}, \cdots, F i l_{f-1}=F i l_{S}, \phi\right),
$$

unique up to the choice of $\phi$. Fix one choice of $\phi$ and let $\rho$ be the corresponding representation after Corollary 3.10, As $F_{i l}$ s satisfy Assumption 6.10, it follows from Proposition 6.12 and Corollary 3.11 (ii) that the isomorphism class of $\rho \otimes k$ is independent of the choice of $\phi$. Finally, the rank condition $\leq p-1$ allows us to apply Theorem 2.3 , to conclude the theorem.

\section{Rigidity THEOREM FOR FontAine MODUlES}

Let $X / W$ be a smooth and projective scheme, equipped with a $W$-ample divisor $Z$. To a ( $p$-torsion) Fontaine module $(H, \nabla, F i l, \Phi)$, one associates naturally the graded Higgs bundle $(E, \theta):=G r_{F i l}(H, \nabla)$ by taking the grading of $(H, \nabla)$ with respect to the filtration $F i l$. In this section, we show that the gr-functor is faithful over those mod $p$-stable objects (with respect to the $\mu_{Z_{1}}$-slope).

For a Griffiths transverse filtration $F i l$ of level $w$, we extend $F i l^{i}=F i l^{0}$ for $-i \in \mathbb{N}$ and $F i l^{w+j}=0$ for $j \in \mathbb{N}$. 
Lemma 7.1. Let $Y$ be a smooth projective variety over an algebraically closed field $k$ and let $(V, \nabla)$ be a flat bundle over $Y$. If there exists a Griffiths transverse filtration Fil on $(V, \nabla)$ such that the associated graded Higgs module $(E, \theta)$ is stable, then it is unique up to a shift of index.

Proof. Suppose on the contrary that there is another gr-semistable filtration $\overline{F i l}$ on $V$ which differs from Fil after arbitrary index shifting. Set $(\bar{E}, \bar{\theta}):=$ $\operatorname{Gr}_{\overline{F i l}}(V, \nabla)$.

Case 1: Suppose that there exists an integer $N$, such that for every $i, F i l^{i} \subset$ $\overline{F i l}{ }^{i+N}$. Also, we can assume $N$ is so chosen that for some $i_{0}, F i l^{i_{0}} \subsetneq F i l^{i_{0}+N+1}$. Then the inclusion induces a natural morphism of Higgs bundles

$$
f:(E, \theta) \rightarrow(\bar{E}, \bar{\theta}) .
$$

Clearly, the morphism $f$ cannot be injective at each closed point. Otherwise, it implies that $F i l^{i}=\bar{F} i l^{i+N}$ for all $i$ which contradicts the assumption that the two previous filtrations are nonequal. So $f$ is not injective. Neither is $f$ zero, since, otherwise, it would imply that $F i l^{i} \subset \bar{F} i l^{i+N+1}$ for all $i$ which contradicts the assumption on $N$. Therefore, on the one hand, as $\operatorname{Im}(f)$ is a quotient of $(E, \theta)$, $\mu(\operatorname{Im}(f))>\mu(E)=\mu(V)$; on the other hand, $\operatorname{Im}(f)$ is also a subobject of $(\bar{E}, \bar{\theta})$, $\mu(\operatorname{Im}(f)) \leq \mu(\bar{E})=\mu(V)$. A contradiction.

Case 2: Otherwise, let $a$ be the largest integer such that $F i l^{a}$ is not contained in $\overline{F i l} l^{a}$, and $b$ the largest integer such that $F i l^{a-i}$ is contained in $\bar{F} i l^{b-i}$ for all $i \geq 0$. Certainly $a>b$. Then we define a morphism of Higgs bundles $f:(E, \theta) \rightarrow(\overline{\bar{E}}, \bar{\theta})$ as follows: for $i>0,\left.f\right|_{E^{a+i}}=0$, and for $j \geq 0,\left.f\right|_{E^{a-j}}$ is given by

$$
E^{a-j}=F i l^{a-j} / F i l^{a-j+1} \rightarrow \bar{F} i l^{b-j} / \bar{F} i l^{b-j+1} .
$$

This is well defined as $F i l^{a+1} \subset \overline{F i} l^{a+1} \subset \bar{F} i l^{b+1}$ and $F i l^{a-j} \subset \bar{F} i l^{b-j}$ for $j \geq 0$. Clearly $f$ cannot be injective. Also, $f$ cannot be zero. Otherwise, one would get the relation $F i l^{a-j} \subset \overline{F i l} l^{b+1-j}$ for all $j \geq 0$, which contradicts the maximality of $b$. The remaining argument goes exactly as Case 1 .

The above statement, true for any characteristic, has the following nice consequence in the current setting.

Proposition 7.2. Let $(E, \theta)$ be a Higgs bundle over $X_{1}$. Suppose $(E, \theta)$ is stable and one-periodic. Then there is a unique one-periodic Higgs-de Rham flow with the initial term $(E, \theta)$ up to isomorphism.

Proof. Let $(E, \theta, F i l, \phi)$ be a one-periodic Higgs-de Rham flow. First, because of the stability assumption on $(E, \theta)$, it follows from Lemma 7.1 that the Hodge filtration $F i l$ on $C_{1}^{-1}(E, \theta)$ is unique up to a possible shift of index. However, $\phi$ is an isomorphism of graded Higgs modules, Fil has to be unique. Second, for any other choice $\varphi$ making $(E, \theta, F i l, \varphi)$ periodic, the composite $\varphi \circ \phi^{-1}$ is an automorphism of $(E, \theta)$. Since it is stable, one must have $\varphi=\lambda \phi$ for a nonzero 
constant $\lambda \in k$. And there is an obvious isomorphism in the category $H D F$ :

$$
(E, \theta, F i l, \phi) \cong(E, \theta, F i l, \lambda \phi),
$$

the proposition follows.

An $\mathbb{F}_{p}$-representation $\rho$ of $\pi_{1}\left(X_{K}\right)$ is said to be absolutely irreducible if the $k$ representation $\rho \otimes k$ is irreducible. A direct consequence of the previous proposition is the following

Corollary 7.3. Notation as above. There is a natural equivalence of categories between the category of absolutely irreducible crystalline $\mathbb{F}_{p}$-representations of $\pi_{1}\left(X_{K}\right)$ with Hodge-Tate weights $\leq p-2$ and the category of one-periodic stable Higgs bundles in $H I G_{p-2}\left(X_{1}\right)$.

Proof. Proposition 7.2 embeds the category of one-periodic stable Higgs bundles over $X_{1}$ into the category of one-periodic Higgs-de Rham flows over $X_{1}$ as a full subcategory. Under the Higgs correspondence and Fontaine-Laffaille-Faltings correspondence (Proposition 3.3 and Theorem 2.3), the category of one-periodic stable Higgs bundles in $H I G_{p-2}\left(X_{1}\right)$ corresponds to a full subcategory of crystalline $\mathbb{F}_{p}$-representations of $\pi_{1}\left(X_{K}\right)$. The image is characterized by the absolutely irreducibility of the associated $\mathbb{F}_{p}$-representations, as shown by [28, Theorem 1.3].

Remark 7.4. Over $\mathbb{C}$, the gr-functor is an equivalence of categories between the category of irreducible complex polarized variations of Hodge structure and the category of stable graded Higgs bundles. The above corollary is a characteristic $p$-analogue of this equivalence. However, compared with the transcendental nature of the quasi-inverse functor in the complex case, the one in the characteristic $p$ case is much more constructive: indeed, it has already been noticed by Ogus-Vologodsky (see [25, Definition 4.16] and the paragraph thereafter) that the flat bundle $(H, \nabla)$ of a strict $p$-torsion Fontaine module is reconstructed by $C_{1}^{-1}(E, \theta)$, no matter whether $(E, \theta)$ stable or not (but it is at least semistable as explained in Proposition 6.3). This point has also been explicitly emphasized in $\S 4$ [13. Furthermore, in Theorem A.4 Appendix A, we show how to construct a gr-semistable filtration on $(H, \nabla)$. By the proof of Lemma 7.1, it has to coincide with the Hodge filtration Fil by a possible shift of index, which is also uniquely determined by the gradings in $E$. The construction of the relative Frobenius from an isomorphism $G r_{F i l}(H, \nabla) \cong(E, \theta)$ is the major content of the functor $\mathcal{C}^{-1}$ in the proof of Proposition 3.3 .

The above results may be lifted to $W_{n}$ for $n$ arbitrary.

Proposition 7.5. Let $(E, \theta)$ be the initial term of a one-periodic Higgs-de Rham flow over $X_{n}$. If the mod $p$ reduction of $(E, \theta)$ is stable over $X_{1}$, then there is a unique one-periodic Higgs-de Rham flow for $(E, \theta)$ up to isomorphism.

Proof. We prove by induction on $n$. The $n=1$ case is Proposition 7.2, We show make the induction hypothesis as follows: let $(\bar{E}, \bar{\theta})$ be the $\bmod p^{n-1}$-reduction of $(E, \theta)$, and $(\bar{E}, \bar{\theta}, \bar{F} i l, \bar{\psi})$ be a one-periodic Higgs-de Rham flow over $X_{n-1}$. Then $F i l$ is the unique lifting of the Hodge filtration in the characteristic $p$ and $\bar{\psi}$ is the unique lifting of the isomorphism in characteristic $p$ up to a scalar in $W_{n-1}$. 
Now let $\left(E, \theta, F i l_{i}, \psi_{i}\right), i=1,2$ be two one-periodic Higgs-de Rham flow over $X_{n}$ lifting $(\bar{E}, \bar{\theta}, \overline{F i l}, \bar{\psi})$ over $X_{n-1}$. It suffices to show the following claims:

(i) $F i l_{1}=F i l_{2}$;

(ii) $\psi_{1}=\lambda \psi_{2}, \lambda \in W_{n}$.

Without loss of generality, we may assume that $F i l$ is reduced. Assume the contrary of the statement (i). Let $a$ be the largest integer such that $F i l_{1}^{a}$ differs from $F i l_{2}^{a}$, and then $b$ be the largest integer such that $F i l_{1}^{a-i} \subseteq F i l_{2}^{b-i}$ for each $i \geq 0$.

Case 1. $b>a$. Then as

$$
F i l_{1}^{a} \subset F i l_{2}^{b} \subset F i l_{2}^{a+1}=F i l_{1}^{a+1}
$$

and $F i l_{1}^{a+1} \subset F i l_{1}^{a}$, we get $F i l_{1}^{a+1}=F i l_{1}^{a}$, and in particular, $\overline{F i l} l^{a+1}=F i l^{a}$. A contradiction.

Case 2. $\quad b=a$. As $F i l_{1}^{a} \subset F i l_{2}^{a}$ and $F i l_{1}^{a}=F i l_{2}^{a} \bmod p^{n-1}$, it follows that $F i l_{1}^{a}=F i l_{2}^{a}$. A contradiction.

Case 3. $b<a$. Let $(H, \nabla)=C_{n}^{-1}(E, \theta)$. We define a morphism

$$
f: G r_{F i l_{1}}(H, \nabla) \rightarrow G r_{F i l_{2}}(H, \nabla)
$$

as follows: for $i>0, f$ on the factor $F i l_{1}^{a+i} / F i l_{1}^{a+i+1}$ is simply zero; for $i \leq 0$, $f: F i l_{1}^{a+i} / F i l_{1}^{a+i+1} \rightarrow F i l_{2}^{b+i} / F i l_{2}^{b+i+1}$ is the natural morphism. It is easy to check that this gives a morphism of Higgs bundles. Because of the choice of $b$, $f$ is nonzero. As its mod $p^{n-1}$ reduction is clearly the zero map, we obtain a nonzero morphism $\frac{f}{\left[p^{n-1}\right]}$ on the mod $p$-reductions of both sides of the morphism $f$, which are isomorphic to the stable Higgs bundle $(E, \theta)_{1}$ in characteristic $p$. Clearly, it is neither an isomorphism. A contradiction. Therefore, $F i l_{1}=F i l_{2}$, and $\operatorname{Gr}_{F i l_{1}}(H, \nabla)=G r_{F i l_{2}}(H, \nabla)$. We continue to show the statement (ii). For this, we consider the composite

$$
\phi:=\psi_{1} \circ \psi_{2}^{-1}:(E, \theta) \cong(E, \theta) .
$$

By the induction hypothesis, $\phi \bmod p^{n-1}=\bar{\lambda} \in W_{n-1}$. Take any lifting $\lambda \in W_{n}$ of $\bar{\lambda}$ and consider the endomorphism $\phi^{\prime}:=\phi-\lambda$ of $(E, \eta)$. As $\phi^{\prime} \bmod p^{n-1}=0$, we get an endomorphism of the stable Higgs bundle in characteristic $p$ :

$$
\frac{\phi^{\prime}}{\left[p^{n-1}\right]}:(E, \theta)_{1} \rightarrow(E, \theta)_{1}
$$

which has to be a scalar $\mu \in k$. So we get $\phi=\lambda+p^{n-1} \mu \in W_{n}$, and the statement (ii) follows.

The following rigidity theorem for Fontaine modules follows immediately from the previous proposition and the Higgs correspondence.

Corollary 7.6. Let $\left(H_{i}, \nabla_{i}, F i l_{i}, \Phi_{i}\right), i=1,2$ be two Fontaine modules (torsion free or not) over $X / W$, and $\left(E_{i}, \theta_{i}\right)$ the associated graded Higgs bundles. If $\left(E_{1}, \theta_{1}\right)$ is isomorphic to $\left(E_{2}, \theta_{2}\right)$ and mod $p$ Higgs stable, then $\left(H_{i}, \nabla_{i}, F i l_{i}, \Phi_{i}\right), i=$ 1,2 are isomorphic. 
Combining Proposition 7.5 with Theorem 5.3 and Corollary 7.3 , we obtain the following

Corollary 7.7. There is a natural equivalence of categories between the category of crystalline $\mathbb{Z}_{p}$ (resp. $W_{n}\left(\mathbb{F}_{p}\right)$ ) representations of $\pi_{1}\left(X_{K}\right)$ with Hodge-Tate weight $\leq p-2$ whose mod $p$ reduction is absolutely irreducible and the category of one-periodic Higgs bundles over $X / W$ (resp. $X_{n} / W_{n}$ ) whose exponent is $\leq p-2$ and $\bmod p$ reduction is stable.

\section{Appendix A. Semistable Higgs bundles of small Ranks are STRONGLY HigGS SEMISTABLE}

Guitang Lan, Mao Sheng, Yanhong Yang and Kang Zuo

In this appendix, we shall prove the following result.

Theorem A.1. Let $(E, \theta)$ be a nilpotent semistable Higgs module over a smooth projective variety $X$ defined over $\overline{\mathbb{F}}_{p}$. If rank $E \leq p$, then $(E, \theta)$ is strongly semistable.

This same result has also been obtained by A. Langer independently. See [19, Theorem 5.12]. In the early version of the manuscript [14], we have proposed the following conjecture which inspired the above theorem.

Conjecture A.2. [14, Conjecture 2.8] A nilpotent semistable Higgs module of exponent $\leq p$ is strongly Higgs semistable.

We had shown the rank two case in [14]. See [14, Theorem 2.6] which is just Proposition 6.4 in the current version. Shortly after the appearance of [14], Lingguang Li [22] has verified the conjecture in the rank three case. The conjecture requires modification in order to be true in the higher rank case.

The key step in the proof is Theorem A.4, a positive characteristic generalization of Simpson's result [33, Theorem 2.5], which states that over a complex smooth projective curve, every vector bundle with an integrable holomorphic connection admits a Griffiths transverse filtration, such that the associated-graded Higgs bundle is semistable. This generalization is proved similarly as in [33. Theorem $2.5]$.

Throughout the appendix, we assume that $Y$ is a smooth projective variety over an algebraically closed field $k, H$ is an ample divisor of $Y$, Higgs modules as well as flat modules are torsion free, and the semistability is referred to the $\mu=\mu_{H^{-}}$ semistability.

Definition A.3. A flat module $(V, \nabla)$ is called $\nabla$-semistable if for every submodule $V_{1} \subset V$ with $\nabla\left(V_{1}\right) \subset V_{1} \otimes_{\mathcal{O}_{Y}} \Omega_{Y}, \mu\left(V_{1}\right) \leq \mu(V)$ holds.

The goal of this section is to prove the following theorem.

Theorem A.4. Let $(V, \nabla)$ be a $\nabla$-semistable flat module over a smooth projective variety $Y$ over an algebraically closed field $k$. Then there exists a Griffiths transverse filtration Fil such that the associated-graded Higgs module to $(V, \nabla, F i l)$ is semistable. 
A Griffiths transverse filtration on $(H, \nabla)$ with semistable graded Higgs module is said to be gr-semistable.

Remark A.5. By [34], every holomorphic vector bundle that admits a connection is of degree 0 , thus in this case every flat bundle $(V, \nabla)$ is automatically $\nabla$ semistable. So the above result generalizes [33, Theorem 2.5]. On the other hand, the $\nabla$-semistability condition in the statement is indeed necessary for its truth over a general field. Let $k$ be a field of characteristic $p \geq 3$ and $V=\mathcal{O} \oplus \mathcal{O}(p)$ be the rank two vector bundle over $\mathbb{P}_{k}^{1}$, equipped with the canonical connection $\nabla_{c a n}$ of the Cartier descent theorem. Then $\left(V, \nabla_{c a n}\right)$ admits no gr-semistable filtration.

To prove the above theorem, we need some lemmas.

Lemma A.6. Let $(E, \theta)$ be a graded Higgs module on $Y$. If $(E, \theta)$ is unstable as a Higgs module, then its maximal destabilizing subsheaf $I \subset E$ is saturated, and it is a sub graded Higgs module, that is $I=\bigoplus_{i=0}^{n} I^{i}$ with $I^{i}:=I \cap E^{i}$.

Proof. The saturated property of the Higgs subsheaf $I \subset E$ follows from the maximality. To show the second property, one chooses $t \in k$ such that $t^{i} \neq 1$ for $0<i \leq n$. Note that there is an isomorphism $f:(E, \theta) \rightarrow\left(E, \frac{1}{t} \theta\right)$ given by $\left.f\right|_{E^{i}}=t^{i} \mathrm{Id}$. Because of the uniqueness of the maximal destabilizing subobject, we see that $f(I)=I$. Let $s$ be any local section of $I$. Write $s$ as $\sum_{i=0}^{n} s^{i}$, where $s^{i}$ is a local section of $E^{i}$. Then for $j \geq 0$,

$$
f^{j}(s)=\sum_{i=0}^{n} t^{j i} s^{i} \in I .
$$

Consider

$$
\left(\begin{array}{c}
s \\
f(s) \\
\vdots \\
f^{n}(s)
\end{array}\right)=\left(\begin{array}{ccccc}
1 & 1 & 1 & \cdots & 1 \\
1 & t & t^{2} & \cdots & t^{n} \\
\vdots & \vdots & \vdots & \ddots & \vdots \\
1 & t^{n} & t^{2 n} & \cdots & t^{n^{2}}
\end{array}\right) \cdot\left(\begin{array}{c}
s^{0} \\
s^{1} \\
\vdots \\
s^{n}
\end{array}\right) .
$$

By assumption on $t$, the coefficient matrix is invertible; thus all $s^{i}$ s are local sections of $I$ and $I=\oplus_{i=0}^{n} I \cap E^{i}$.

Let $(V, \nabla)$ be a flat module over $Y$. Start with an arbitrary Griffiths transverse filtration Fil of level $n$ and consider the associated Higgs module $\left(\operatorname{Gr}_{F i l}(V), \theta\right)$. If $\left(\operatorname{Gr}_{F i l}(V), \theta\right)$ is unstable, let $I_{F i l}$ be its maximal destabilizing subobject. By Lemma A.6,

$$
I_{F i l}=\oplus_{i=0}^{n} I_{F i l}^{i}, \quad I_{F i l}^{i} \subset F i l^{i} / F i l^{i+1} \subset V / F i l^{i+1} .
$$

Following the construction of Simpson (see $\S 3[33]$ ), we define an operation $\xi$ on the set of Griffiths transverse filtrations. The new filtration $\xi(F i l)$ of $V$ is given by

$$
\xi(F i l)^{i+1}:=\operatorname{Ker}\left(V \rightarrow \frac{V / F i l^{i+1}}{I_{F i l}^{i}}\right), \text { for } 0 \leq i \leq n ; \quad \xi(F i l)^{0}=V
$$


Note that $F i l^{i} \supset \xi(F i l)^{i+1} \supset F i l^{i+1}$, and there is a short exact sequence:

$$
0 \rightarrow \operatorname{Gr}_{F i l}^{i}(V) / I_{F i l}^{i} \rightarrow \operatorname{Gr}_{\xi(F i l)}^{i}(V) \rightarrow I_{F i l}^{i-1} \rightarrow 0, \quad \text { for } 0 \leq i \leq n+1 .
$$

Adding altogether, we obtain a short exact sequence of graded Higgs modules:

$$
0 \rightarrow \operatorname{Gr}_{F i l}(V) / I_{F i l} \rightarrow \operatorname{Gr}_{\xi(F i l)}(V) \stackrel{h}{\rightarrow} I_{F i l}^{[1]} \rightarrow 0
$$

where $E^{[k]}$ denotes for the graded Higgs module $E$ with index shifted so that $\left(E^{[k]}\right)^{i}=E^{i-k}$. If $(E, \theta)$ is unstable, let $\mu_{\max }(E)$ and $r_{\max }(E)$ denote respectively the slope and rank of the maximal destabilizing subobject of $E$; otherwise, let $\mu_{\max }(E)=\mu(E)$ and $r_{\max }(E)=\operatorname{rk}(E)$. By (A.6.4), we have

Lemma A.7. The following statements hold:

(1) $\mu_{\max }\left(G r_{\xi(\text { Fil })}(V)\right) \leq \mu_{\max }\left(G r_{F i l}(V)\right)$.

(2) If $\mu_{\max }\left(G r_{\xi(F i l)}(V)\right)=\mu_{\max }\left(G r_{F i l}(V)\right)$, then

$$
r_{\max }\left(G r_{\xi(\text { Fil })}(V)\right) \leq r_{\max }\left(G r_{F i l}(V)\right) \text {. }
$$

(3) Furthermore, if $r_{\max }\left(G r_{\xi(F i l)}(V)\right)=r_{\max }\left(G r_{F i l}(V)\right)$, then the composite $\operatorname{map} I_{\xi(F i l)} \rightarrow G r_{\xi(F i l)}(V) \rightarrow I_{F i l}^{[1]}$ is injective and is an isomorphism outside a codimension two closed subset of $Y$, where $I_{\xi(F i l)}$ is the maximal destabilizing subobject of $G r_{\xi(F i l)}(V)$.

Proof. The proof follows from the following exact sequence:

$$
0 \rightarrow \operatorname{Ker}(h) \cap I_{\xi(F i l)} \rightarrow I_{\xi(\text { Fil })} \rightarrow h\left(I_{\xi(\text { Fil })}\right) \rightarrow 0,
$$

which is induced from (A.6.4 $)$, and where $h\left(I_{\xi(F i l)}\right)$ is a subsheaf of $I_{F i l}^{[1]}$.

The following lemma is the key to our theorem.

Lemma A.8. Let Fil be a Griffiths transverse filtration of level $n$ on a flat module $(V, \nabla)$. Assume $(V, \nabla)$ to be $\nabla$-semistable. Then if the associated-graded Higgs module $\left(G_{F i l}(V), \theta\right)$ is unstable, then at least one of the following two strict inequalities holds:

$\mu_{\max }\left(G r_{\xi^{n+1}(\text { Fil })}(V)\right)<\mu_{\max }\left(G r_{F i l}(V)\right) ; \quad r_{\max }\left(G r_{\xi^{n+1}(F i l)}(V)\right)<r_{\max }\left(G r_{F i l}(V)\right)$.

Proof. Put $\mu_{k}=\mu_{\max }\left(\mathrm{Gr}_{\xi^{k}(\text { Fil })}(V)\right)$ and $r_{k}=r_{\max }\left(\mathrm{Gr}_{\xi^{k}(\text { Fil })}(V)\right)$ for $k \geq 0$. By Lemma A.7 (1)-(2), $\left(\mu_{k}, r_{k}\right)$ decreases in the lexicographic ordering when $k$ grows. Argue by contradiction. Suppose on the contrary that $\mu_{n+1}=\mu_{0}$ and $r_{n+1}=r_{0}$. Then, for $0 \leq k \leq n, \mu_{k+1}=\mu_{k}$ and $r_{k+1}=r_{k}$, and, by Lemma A.7 (3), $I_{\xi^{k+1}(\text { Fil })} \subseteq I_{\xi^{k}(\text { Fil })}^{[1]} \subseteq I_{\text {Fil }}^{[k+1]}$, which are locally free and coincide with each other away from a codimension two closed subset $Z \subset Y$. Hence they have the same slope.

A direct calculation on the short exact sequences (A.6.4) for $G r_{\xi^{k+1}(\text { Fil })}(V)$, for $k$ running from 0 to $n$, reveals the following fact: the short exact sequence (A.6.4) of graded Higgs modules for $\operatorname{Gr}_{\xi^{n+1}(\text { Fil })}(V)$ takes a special form:

$$
0 \rightarrow \oplus_{i=0}^{n} E^{i} \rightarrow \oplus_{i=0}^{2 n+1} E^{i} \rightarrow \oplus_{i=n+1}^{2 n+1} E^{i} \rightarrow 0
$$


where

$$
G r_{\xi^{n+1}(F i l)}(V)=G r_{\xi^{n+1}(F i l)}(V)=\oplus_{i=0}^{2 n+1} E^{i},
$$

and $G r_{\xi^{n}(F i l)}(V) / I_{\xi^{n}(F i l)}=\oplus_{i=0}^{n} E^{i}$ and $I_{\xi^{n}(F i l)}^{[1]}=\oplus_{i=n+1}^{2 n+1} E^{i}$. Since over the open subset $U=Y-Z$,

$$
\left.I_{\xi^{n}(F i l)}^{[1]}\right|_{U}=\left.\left.I_{\xi^{n+1}(F i l)}\right|_{U} \subset G r_{\xi^{n+1}(F i l)}(V)\right|_{U},
$$

it follows that the sequence (A.6.4) splits over $U$ as graded Higgs modules. Thus, one has

$$
\left.\theta\right|_{U}\left(\left.E^{n+1}\right|_{U}\right)=0
$$

Hence, $\theta$ is indeed zero on $E^{n+1}$, which means nothing but $V^{\prime}:=\left(\xi^{n+1}(F i l)\right)^{n+1}$ is $\nabla$-invariant. On the other hand, one has the relation that

$$
\mu\left(V^{\prime}\right)=\mu\left(G r_{\xi^{n+1}(\text { Fil })}\left(V^{\prime}\right)\right)=\mu\left(I_{\xi^{n}(\text { Fil })}^{[1]}\right)=\mu\left(I_{F i l}^{[n+1]}\right)=\mu\left(I_{F i l}\right)>\mu(V) .
$$

The strict inequality contradicts the $\nabla$-semistability of $(V, \nabla)$. This completes the lemma.

Proof of Theorem A.4. One takes an arbitrary Griffiths transverse filtration Fil of $(V, \nabla)$ (e.g. the trivial filtration), and then applies consecutively the operator $\xi$ on Fil. The meanings of $\mu_{k}$ and $r_{k}$ for $k \geq 0$ are the same as those in the previous lemma. Lemma A.7 says that pairs $\left(\mu_{k}, r_{k}\right), k \geq 0$ decrease in the lexicographic ordering as $k$ grows. So for certain $k_{0} \geq 0$, the sequence $\left\{\left(\mu_{k}, r_{k}\right)\right\}_{k \geq k_{0}}$ becomes constant. Then Lemma A.8 asserts that $\xi^{k_{0}}(F i l)$ has to be a gr-semistable filtration of $(V, \nabla)$.

Remark A.9. In the proof of Theorem A.4, if we start with the trivial filtration $V=F i l^{0} \supset F i l^{1}=0$, the resulting gr-semistable filtration has the extra property that it is invariant under any automorphism and hence has the same definition field as $(V, \nabla)$. In a Griffiths transverse filtration $F i l: V=F i l^{0} \supseteq F i l^{1} \supseteq \cdots$ on $(V, \nabla)$, we call a term $F i l^{i}, i \geq 1$ redundant if $F i l^{i-1}=F i l^{i}$. One can remove all redundant terms from the filtration and shift the indices correspondingly so that the resulting filtration is a strictly decreasing filtration of form $F i l_{\text {red }}: V=$ $F i l^{0} \supsetneq F i l^{1} \supsetneq F i l^{2} \supsetneq \cdots$. We call this operation the reduction of a filtration, and a filtration reduced if it is equal to its reduction. It is not hard to observe that $G r_{F i l}(V, \nabla)$ and $G r_{F i l_{r e d}}(V, \nabla)$ are isomorphic as Higgs modules. Thus, the reduction of a gr-semistable filtration on $(V, \nabla)$ is again gr-semistable.

Proof of Theorem A.1. Note first that the inverse Cartier transform $(V, \nabla)$ of a Higgs module $(E, \theta)$ is $\nabla$-semistable if and only if $E$ is $\theta$-semistable. This is a direct consequence of the equivalence theorem of the (inverse) Cartier transform of Ogus-Vologodsky [25]. So for a nilpotent semistable Higgs module of rank $\leq p$, its inverse Cartier transform is a $\nabla$-semistable flat module of rank $\leq p$. By Theorem A.4 and Remark A.9, there exists a Griffiths transverse filtration Fil on $(V, \nabla)$ (of level $\leq p-1$ for the reason of rank) such that $\operatorname{Gr}_{F i l}(V, \nabla)$ is nilpotent semistable of the same rank $\leq p$ and defined over the same ground field of $(E, \theta)$. Therefore, we can obtain a semistable Higgs-de Rham flow with the leading term $(E, \theta)$ by applying the previous construction inductively. This completes the proof. 
Acknowledgements: Christopher Deninger has drawn our attention to the work [18], Adrian Langer has helped us understanding [18] and Carlos Simpson has explained us the proof of [33, Proposition 4.3]. We also appreciate several e-mail communications with Mark Kisin. We thank them heartily. We would like to thank especially an anonymous referee who has pointed out several drawbacks in the original draft and also provided us a very constructive suggestion in the construction of the lifting of the inverse Cartier transform over a truncated Witt ring. The second approach in the construction of the functor $\mathcal{T}_{n}$ in $\S 4$ is due to the referee.

\section{REFERENCES}

[1] P. Deligne, Un théorème de finitude pour la monodromie, Discrete groups in geometry and analysis (New Haven, Conn., 1984), 1-19, Progr. Math., 67, Birkhäuser Boston, Boston, MA, 1987.

[2] C. Deninger, A. Werner, Vector bundles on $p$-adic curves and parallel transport, Ann. Scient. Éc. Norm. Sup. 38 (2005), 553-597.

[3] M. Emerton, M. Kisin, The Riemann-Hilbert correspondence for unit F-crystals, Astérisque No. 293 (2004), vi+257 pp.

[4] G. Faltings, Crystalline cohomology and $p$-adic Galois-representations, Algebraic analysis, geometry, and number theory (Baltimore, MD, 1988), 25-80, Johns Hopkins Univ. Press, Baltimore, MD, 1989.

[5] G. Faltings, Integral crystalline cohomology over very ramified valuation rings, Journal of the AMS, Vol. 12, no. 1, 117-144, 1999.

[6] G. Faltings, A p-adic Simpson correspondence, Advances in Mathematics 198 (2005), 847-862.

[7] J.-M. Fontaine, G. Laffaille, Construction de représentation $p$-adiques, Ann. Sci. Éc. Norm. Sup. 15 (1982), 547-608.

[8] J.-M. Fontaine, W. Messing, p-adic periods and p-adic étale cohomology, Current trends in arithmetical algebraic geometry (Arcata, Calif., 1985), 179-207, Contemp. Math., 67, Amer. Math. Soc., Providence, RI, 1987.

[9] P. Griffiths, Periods of integrals on algebraic manifolds. III. Some global differentialgeometric properties of the period mapping, Inst. Hautes Études Sci. Publ. Math. No. $38,1970,125-180$.

[10] N. Hitchin, The self-duality equations on a Riemann surface, Proc. London Math. Soc. (3), 55, 1987, 59-126.

[11] N. Katz, Travaux de Dwork, Séminaire Bourbaki, 24ème année (1971/1972), Exp. No. 409, pp. 167-200. Lecture Notes in Math., Vol. 317, Springer, Berlin, 1973.

[12] N. Katz, $P$-adic properties of modular schemes and modular forms, Lecture Notes in Mathematics, Vol. 350, Springer, Berlin, 1973.

[13] G.-T. Lan, M. Sheng, K. Zuo, Nonabelian Hodge theory in positive characteristic via exponential twisting, Mathematical Research Letters, 22 (2015), no. 3, 859-879.

[14] G.-T. Lan, M. Sheng, K. Zuo, Semistable Higgs bundles and representations of algebraic fundamental groups: Positive characteristic case, arxiv 1210.8280v1, 2012.

[15] G.-T. Lan, M. Sheng, Y.-H. Yang, K. Zuo, Semistable Higgs bundles of small ranks are strongly Higgs semistable, arXiv:1311.2405, 2013.

[16] G.-T. Lan, M. Sheng, Y.-H. Yang, K. Zuo, Uniformization of $p$-adic curves via Higgs-de Rham flows, J. reine angew. Math., ISSN (Online) 1435-5345, ISSN (Print) 0075-4102, DOI:https://doi.org/10.1515/crelle-2016-0020, July 2016.

[17] A. Langer, Semistable sheaves in positive characteristic, Annals of Mathematics 159 (2004), 241-276. 
[18] A. Langer, Moduli spaces of sheaves in mixed characteristic, Duke Math. J. 124 (2004), no. $3,571-586$.

[19] A. Langer, Semistable modules over Lie algebroids in positive characteristic, Documenta Mathematica 19 (2014) 561-592.

[20] A. Langer, Bogomolov's inequality for Higgs sheaves in positive characteristic, Invent. Math., 199 (2015), 889-920.

[21] H. Lange, U. Stuhler, Vektorbündel auf Kurven und Darstellungen der algebraischen Fundamentalgruppe, Math. Z. 156 (1977), 73-83.

[22] L.-G. Li, On a conjecture of Lan-Sheng-Zuo on semistable Higgs bundles: rank 3 case, Internat. J. Math. 25 (2014), no. 2, 1450013.

[23] S. Mochizuki, A theory of ordinary p-adic curves, Publ. Res. Inst. Math. Sci. 32 (1996), no. $6,957-1152$.

[24] A. Ogus, F-crystals, Griffiths transversality, and the Hodge decomposition, Astérisque No. 221 (1994).

[25] A. Ogus, V. Vologodsky, Nonabelian Hodge theory in characteristic $p$, Publ. Math. Inst. Hautes études Sci. 106 (2007), 1-138.

[26] M. Sheng, X. He, K. Zuo, A note on the characteristic $p$ nonabelian Hodge theory in the geometric case, International Journal of Mathematics, Vol. 26, No. 1 (2015) 1550011.

[27] M. Sheng, J.-J. Zhang, K. Zuo, Higgs bundles over the good reduction of a quaternionic Shimura curve, J. reine angew. Math., DOI 10.1515, 2011.

[28] M. Sheng, K. Zuo, Periodic Higgs subbundles in positive and mixed characteristic, arXiv:1206.4865v1, 2012.

[29] A. Shiho. Notes on generalizations of local Ogus-Vologodsky correspondence, arXiv: $1206.5907 \mathrm{v} 1,2012$.

[30] C. Simpson, Constructing variations of Hodge structure using Yang-Mills theory and applications to uniformization, J. Amer. Math. Soc. 1 (1988), no. 4, 867-918.

[31] C. Simpson, Higgs bundles and local systems, Inst. Hautes Études Sci. Publ. Math. No. 75 (1992), 5-95.

[32] C. Simpson, Moduli of representations of the fundamental group of a smooth projective variety. I, II, Inst. Hautes Études Sci. Publ. Math. No. 79, 47-129 (1994), No. 80, 5-79 (1995).

[33] C. Simpson, Iterated destabilizing modifications for vector bundles with connections, Vector bundles and complex geometry, 183-206, Contemp. Math., 522, Amer. Math. Soc. Providence, RI. 2010.

[34] A. Weil, Généralisation des fonctions abéliennes. J. Math. Pures Appl. 17 (1938), 4787.

E-mail address: lan@uni-mainz.de; zuok@uni-mainz.de

Institut für Mathematik, Universität Mainz, Mainz, 55099, Germany

E-mail address: msheng@ustc.edu.cn

School of Mathematical Sciences, University of Science and Technology of

China, Hefei, 230026, China 